\title{
Tritonlike Molecules of Three Identical Baryons
}

\author{
Li Ma \\ Department of Physics, Beijing Jiaotong University, Beijing 100044, China \\ Correspondence should be addressed to Li Ma; ma.li@bjtu.edu.cn
}

Received 8 October 2021; Accepted 18 January 2022; Published 14 February 2022

Academic Editor: Shi Hai Dong

Copyright (c) $2022 \mathrm{Li} \mathrm{Ma}$. This is an open access article distributed under the Creative Commons Attribution License, which permits unrestricted use, distribution, and reproduction in any medium, provided the original work is properly cited. The publication of this article was funded by SCOAP ${ }^{3}$.

\begin{abstract}
In nuclear physics, triton and helium-3 nucleus can be understood as three-body hadronic molecules. Analogous to the loosely bound structures for the triton and helium-3 nucleus, whether there is a bound state formed by three hadrons leaves us an open issue. Based on the one-boson exchange model as well as the adoption of the variational approach, we make a comprehensive investigation on the tritonlike systems of three identical baryons $N N N, \Lambda \Lambda \Lambda, \Xi \Xi \Xi$, and $\Sigma \Sigma \Sigma$. We predict that the three-body molecular states for the systems of three identical hadrons of baryon octet are probably existent as long as their two-body subsystems have bound states. The numerical results of this work may be helpful for the theoretical and experimental researches on the trihadron molecules in future.
\end{abstract}

\section{Introduction}

Numerous exotic states called "XYZ" reported in the last few decades pose great challenges to the traditional quark model [1-11]. Some of them sit in the vicinity of the open charm threshold can be viewed as good candidates of di-hadron molecules [12]. The scenario of dihadron molecule was first motivated by the deuteron, in which a proton and a neutron form a loosely bound state by colorless strong interactions. The analog of deuteron as well as the largely accumulated experimental data on the exotic states arouses interest in the explorations on two-body hadronic systems. Currently, lots of effort has been spent on searching for the bound states of two-hadron systems [13-26]. In nuclear physics, triton and helium-3 nucleus can be understood as trihadron molecules, where the three nucleons bind together via colorless strong interactions with the binding energy $8.40 \mathrm{MeV}$ for triton and $7.80 \mathrm{MeV}$ for helium-3 nucleus. Along the same line for deuteron, the existence of triton and helium3 nucleus leaves us an open questions that whether threehadron systems have loosely bound states.

Generally, the Faddeev equation provides us a rigorous tool to explore the bound solution of a three-body system [27-41]. One of the common methods to simplify the Faddeev equation of specific systems is the fixed center approx- imation (FCA). For instance, the study of the $X(2175)$ as a resonance of the $\phi K \bar{K}$ has been performed through the FCA method [42]. The $\pi K \bar{K}$ and $\pi \pi \eta$ via the FCA on the unitary chiral dynamics were also performed [43]. Theoretical studies on the $K K \bar{K}$ [44], the $N K \bar{K}$ [45], the $J / \psi K \bar{K}$ [46], the $N D K$, the $\bar{K} D N$, and $N D \bar{D}$ [47], the $B D D$ and $B D$ $\bar{D}$ [48], and $D \bar{D}^{*} K$ and $\bar{D} D^{*} K[49]$ were published in recent years. Discussions on the $B B^{*} B^{*}$ [50], the $\Xi N N$ [51], and the $\Omega N N$ and $\Omega \Omega N$ [52] with similar method also can be found. Other calculations by using FCA method are listed in Refs [53-63]. Another method for three-body systems is isobar formalism, which has been adopted to discuss the threenucleon system with $\Delta(1236)$ isobar [64]. Some other applications via isobar formalism can be found in Refs [65-68]. The third useful tool to describe a three-body system called dimer formalism was presented in a series of studies, where a composite field is introduced to describe the two-body subsystem when rescattering with a third particle [69-75]. The Gauss Expansion Method is another effective tool for few-body systems [76], which has been applied to discuss few-nucleon systems [77] and the DDDK system [78].

The one-boson exchange (OBE) model works well in describing nuclear force [79]. Among the all OBE models, the Nijmegen OBE model is a very successful one [80-84]. It provides an excellent simultaneous description of the rich 
and accurate nucleon-nucleon $(\mathrm{NN})$ and the more scarce hyperon-nucleon (YN) low-energy scattering data. The Nijmegen model is also successful to describe the hyperonhyperon interactions and hypernuclear systems. In general, the OBE model contains long-range force from $\pi$ and $\eta$ exchange, medium-range force from $\sigma$ exchange, and short-range force from $\rho / \omega / \phi$. There are many theoretical studies on dihadron systems in the framework of the OBE model [22-26]. The OBE interaction with the exchange of $\pi, \eta, \sigma, \rho, \omega$, and $\phi$ plays an important role in the formation of dihadron molecules. One may wonder how the OBE interaction works in a trihadron system and whether the trihadron system has a loosely bound state. As we know, a triton contains one proton and two neutrons. Under the SU(3) chiral symmetry, proton and neutron belong to the octet of the $1 / 2^{+}$baryon. The existence of triton leaves us an interesting question that whether an identical three-body system composed of the other members in the octet of the $1 / 2^{+}$ baryon has a bound state. Since the success of the OBE mechanism in the description of deuteron, it is quite natural to extend the mechanism to a trihadron system consists of three baryons. In the present work we shall perform investigations on the three-body systems composed of the identical hadrons from the octet of the $1 / 2^{+}$baryon. For simplicity, we

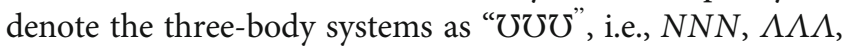
$\Xi \Xi \Xi$, and $\Sigma \Sigma \Sigma$. Other configurations will be studied in a future work.

This work is organized as follows. After the introduction, we present the formalism for an identical trifermion system within the framework of potential interactions in Section 2. We apply our formalism to the three-nucleon system to verify its feasibility in Section 3.2. Then, we extend the formalism to the trihyperon systems composed of three identical hadrons from the octet of the $1 / 2^{+}$baryon for searching their possible molecular states in Sections 3.3-3.5. The last section is brief summary. Some technicalities are relegated to the Appendix.

\section{Formalism}

In this section, we construct the general formalism for an identical trifermion system. As illustrated in Figure 1, we can use $a, b$, and $c$ to label the three fermions, i.e., $\mho_{a} \mho_{b} \mho_{c}$ . Here, the labels are artificial, as the system is invariant under the change of the order of $a, b$, and $c$. Since the system contains three identical fermions, its total wave function should be antisymmetric under exchange of its two constituents. We use $V\left(\vec{r}_{a b}\right), V\left(\vec{r}_{b c}\right)$, and $V\left(\vec{r}_{a c}\right)$ to denote the effective potentials between $\mho_{a}$ and $\mho_{b}, \mho_{b}$ and $\mho_{c}$, and $\mho_{c}$ and $\mho_{a}$, respectively. $\vec{r}_{i j}$ is the relative displacement between the $i$ th and $j$ th fermions. $T_{a}, T_{b}$, and $T_{c}$ are the kinetic energy for the fermions $\mho_{a}, \mho_{b}$, and $\mho_{c}$ in their center-ofmass frame, respectively.

For a better description of the interactions between any two constituents in the system, we divide the channel of $\mho_{a} \mho_{b} \mho_{c}$ into $\left\{\widehat{\mho}_{a} \mho_{b} \mho_{c}, \mho_{a} \widehat{\mho}_{b} \mho_{c}, \mho_{a} \mho_{b} \widehat{\mho}_{c}\right\}$. We make a convention that a fermion $\mho$ absorbs a virtual boson will acquire a "hat" over it. Conversely, a fermion $\widehat{\mho}$ emits a vir-

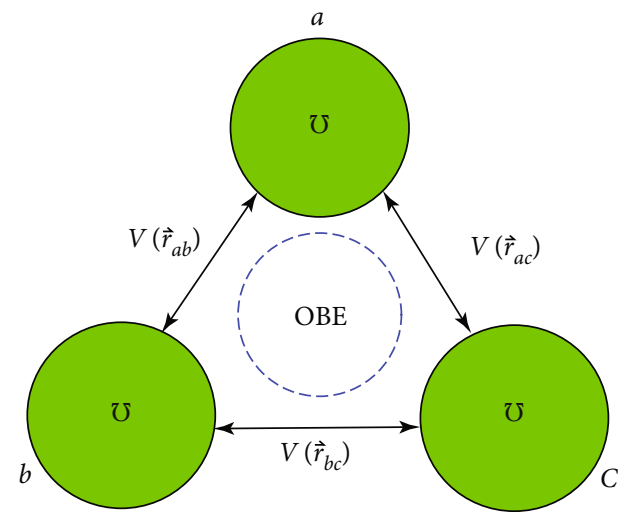

FIgURe 1: Dynamical illustration of the identical trifermion system $\mho \mho \mho$ with a circle describing the delocalized OBE interaction inside.

tual boson will lose its "hat." Within this convention, the divided three-channel space is equivalent to the original channel $\{\mho \mho \mho\}$. The virtual boson is exchanged between any two constituents of the system, and it is not localized between any two constituents but rather is shared by the whole system. It is very similar with the delocalized $\Pi$ bond in benzene molecule where a pair of electrons is shared by the six carbon atoms.

The effective Hamiltonian of the three-fermion system in the channel space $\left|\mho_{B} \mho_{B} \mho_{B}\right\rangle:=\left\{\widehat{\mho}_{a} \mho_{b} \mho_{c}, \mho_{a} \widehat{\mho}_{b} \mho_{c}, \mho_{a}\right.$ $\left.\mho_{b} \widehat{\mho}_{c}\right\}$ takes the following form:

$$
H_{T}=\left[\begin{array}{ccc}
T_{a}+T_{b}+T_{c} & V\left(\vec{r}_{a b}\right) & V\left(\vec{r}_{a c}\right) \\
V\left(\vec{r}_{a b}\right) & T_{a}+T_{b}+T_{c} & V\left(\vec{r}_{b c}\right) \\
V\left(\vec{r}_{a c}\right) & V\left(\vec{r}_{b c}\right) & T_{a}+T_{b}+T_{c}
\end{array}\right],
$$

where $V=V_{\pi}+V_{\eta}+V_{\rho}+V_{\omega}+V_{\phi}+V_{\sigma}$. The $V_{\pi}, V_{\eta}$, $V_{\rho}, V_{\omega}, V_{\phi}$, and $V_{\sigma}$ are the effective potentials from $\pi, \eta$, $\rho, \omega, \phi$, and $\sigma$ exchanges, respectively.

One may wonder whether we can obtain the binding solutions by diagonalizing the Hamiltonian in Equation (1) directly. In principle, we can do it, but the procedure contains lots of complicated integrations when the potentials are some combinations of Yukawa potentials. There are two independent degrees of freedom for the motion of a three-body system which can be expressed as a set of Jacobi coordinates generally. Thus, the Hamiltonian elements will contain integrations in several directions. It is quite hard if the potentials are complicated. Therefore, we should resort an approximation method to simplify the problem. The core question to solve the three-body system is how to handle the effect of one of the particle on the scattering of the other two.

The Born-Oppenheimer approximation works well on the system composed of light particles and heavy particles $[24,85-87]$. Through introducing the Born-Oppenheimer (BO) potential to describe the contribution of light particle 
on the remaining two heavy particles, we can simplify the three-body system into a two-body system. If the BO potential is strong enough, the three-body system may have a bound state. Within this scenario, the light particle works like "glue" to bind the two heavy particles. In fact, the Born-Oppenheimer approximation is a kind of adiabatic approximation that we divided the degrees of freedom of the three-body system into a heavy one and a light one. It inspires us that the $\mathrm{BO}$ potential can reflect the influence of one of the particles on the dynamics of the remaining two.

Even though the application of the $\mathrm{BO}$ approximation is not straightforward for the system of three identical particles, one can still adopt the $\mathrm{BO}$ potential to describe the influence of one of the particles on the scattering of the other two. Since the system $\mho_{a} \mho_{b} \mho_{c}$ is invariant under the interchange of the $a, b$, and $c$, one should count the contribution of each fermion on the dynamics of the other two fermions one by one. Within this scenario, one can simplify the system into three twobody subsystems $\mho_{a} \mho_{b}, \mho_{b} \mho_{c}$, and $\mho_{c} \mho_{a}$ and add the corresponding $\mathrm{BO}$ potential from the remaining one in each subsystem instead. If the three subsystems have a negative common eigenvalue, the whole system may have a threebody bound state. We can call this method as the BornOppenheimer potential method (BOP method) for simplicity [88]. The procedure of the BOP method can be summarized into the step tree shown in Figure 2.

2.1. Born-Oppenheimer Potential. As the discussion above, we can use the $\mathrm{BO}$ potential to describe the influence of one of the fermions on the dynamics of the other two. That is to say, one can separate the $\mho_{a}$ from the three-fermion system and add the $\mathrm{BO}$ potential from the $\mho_{a}$ when exploring the dynamics of the subsystem $\mho_{b} \mho_{c}$. The derivation procedure of the $\mathrm{BO}$ potential is very similar with the $\mathrm{BO}$ approximation. First we investigate the dynamics of the $\mho_{a}$ with the assumption that the distance of the $\mho_{b}$ and $\mho_{c}$ is a fixed parameter. Here, the $\mho_{a}$ has one-boson interactions with the $\mho_{b}$ and $\mho_{c}$ which can be regarded as two static sources. We can derive the binding energy of the $\mho_{a}$ which should be the function of $r_{b c}$. Then, we subtract the binding energy of the $\mho_{a}$ in the limit $r_{b c} \longrightarrow \infty$ which is trivial for

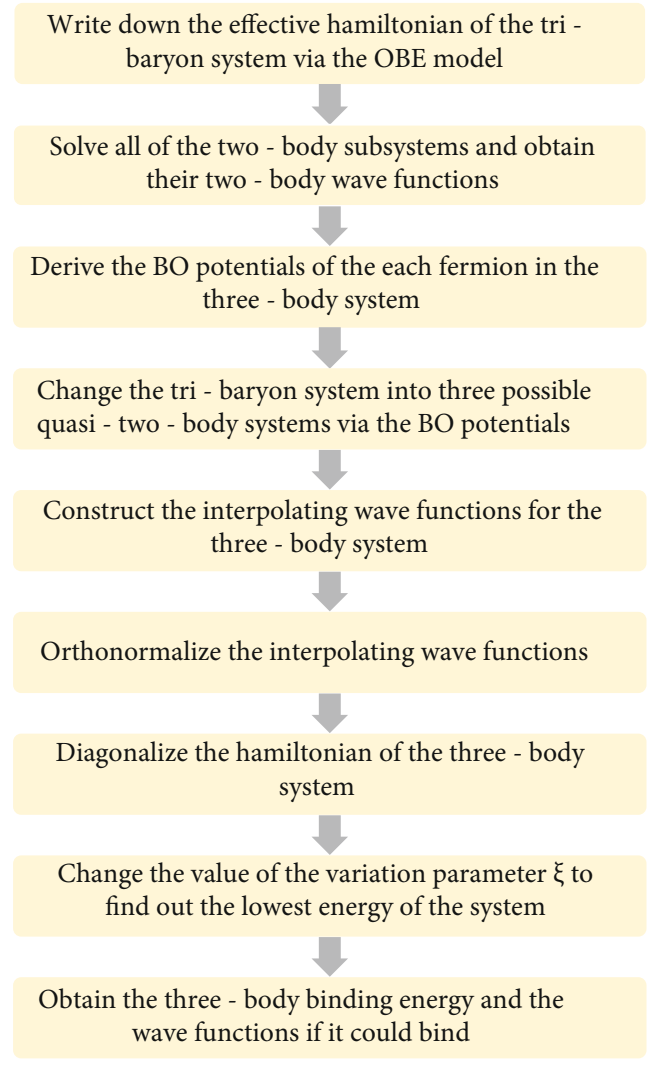

FIGURE 2: Step tree of the BOP method.

the trifermion system. One can separate the effective Hamiltonian in Equation (1) from the fermion $\mho_{a}$,

$$
H_{a}=\left[\begin{array}{ccc}
T_{a b}\left(\text { or } T_{a c}\right) & V_{a b} & V_{a c} \\
V_{a b} & T_{a b}\left(\text { or } T_{a c}\right) & 0 \\
V_{a c} & 0 & T_{a b}\left(\text { or } T_{a c}\right)
\end{array}\right]
$$

and then, the remaining part of the Hamiltonian in Equation (1) is

$$
H_{b c}=H_{T}-H_{a}=\left[\begin{array}{ccc}
T_{a b, c}+T_{t}\left(\text { or } T_{a c, b}+T_{t}\right) & 0 & 0 \\
0 & T_{a b, c}+T_{t}\left(\text { or } T_{a c, b}+T_{t}\right) & V_{b c} \\
0 & V_{b c} & T_{a b, c}+T_{t}\left(\text { or } T_{a c, b}+T_{t}\right)
\end{array}\right],
$$

where we have used the abbreviations $V_{a b}, V_{b c}$, and $V_{a c}$ for $V\left(\vec{r}_{a b}\right), V\left(\vec{r}_{b c}\right)$, and $V\left(\vec{r}_{a c}\right)$, respectively. The abbreviation $T_{a b}$ denotes the relative kinetic energy between the fermion $\mho_{a}$ and $\mho_{b}$, while $T_{a b, c}$ denotes the relative kinetic energy between the fermion $c$ and the two-body center of mass for the fermion $\mho_{a}$ and $\mho_{b}$. The abbreviations $T_{a c}$ and $T_{a c, b}$ have the similar definitions. $T_{t}$ is the kinetic energy of the threebody center of mass which is trivial for our calculations.
Assuming the wave function between the $\mho_{a}$ and $\mho_{b}$ is $\Psi_{2}$, one can write the two-body wave function in the channel space $\left|\mho_{B} \mho_{B}\right\rangle:=\left\{\widehat{\mho}_{a} \mho_{b}, \mho_{a} \widehat{\mho}_{b}\right\}$, which is

$\Psi_{2}=\psi\left(\vec{r}_{a b}\right)\left|\mho_{B} \mho_{B}\right\rangle=\frac{1}{\sqrt{2}} \psi\left(\vec{r}_{a b}\right)\left|\widehat{\mho}_{a} \mho_{b}\right\rangle+\frac{1}{\sqrt{2}} \psi\left(\vec{r}_{a b}\right)\left|\mho_{a} \widehat{\mho}_{b}\right\rangle$. 
The $\psi\left(\vec{r}_{a b}\right)$ satisfies the Schrödinger equation

$$
\left(T_{a b}+V_{a b}\right) \psi_{a b}=E_{2} \psi_{a b}
$$

where the $E_{2}$ is the two-body eigenenergy of the $\mho_{a} \mho_{b}$. Generally, the two-body eigenenergy $E_{2}$ and the two-body wave function $\psi_{a b}$ depend on the specific isospin channel of the two-body subsystem. The abbreviations $\psi_{a b}$ and $\psi_{a c}$ are used for $\psi\left(\vec{r}_{a b}\right)$ and $\psi\left(\vec{r}_{a c}\right)$, respectively. Equation (5) in the channel space $\left|\mho_{B} \mho_{B}\right\rangle:=\left\{\widehat{\mho}_{a} \mho_{b}, \mho_{a} \widehat{\mho}_{b}\right\}$ reads

$$
\left(\begin{array}{cc}
T & V_{a b} \\
V_{a b} & T
\end{array}\right)\left(\begin{array}{l}
\frac{1}{\sqrt{2}} \psi_{a b} \\
\frac{1}{\sqrt{2}} \psi_{a b}
\end{array}\right)=E_{2}\left(\begin{array}{l}
\frac{1}{\sqrt{2}} \psi_{a b} \\
\frac{1}{\sqrt{2}} \psi_{a b}
\end{array}\right)
$$

Given the wave function $\psi_{a b}$, we can obtain the twobody eigenenergy $E_{2}$, which is $E_{2}=\psi_{a b} T_{a b} \psi_{a b}+\psi_{a b} V_{a b} \psi_{a b}$. The final wave function of the $\mho_{a}$ could be the superposition of the two components

$$
\begin{aligned}
\psi_{a}\left(\vec{r}_{a b}, \vec{r}_{a c}\right)= & N\left\{\left[\frac{1}{\sqrt{2}} \psi\left(\vec{r}_{a b}\right)+\frac{1}{\sqrt{2}} \psi\left(\vec{r}_{a c}\right)\right]\left|\widehat{\mho}_{a} \mho_{b} \mho_{c}\right\rangle\right. \\
& +\frac{1}{\sqrt{2}} \psi\left(\vec{r}_{a b}\right)\left|\mho_{a} \widehat{\mho}_{b} \mho_{c}\right\rangle \\
& \left.+\frac{1}{\sqrt{2}} \psi\left(\vec{r}_{a c}\right)\left|\mho_{a} \mho_{b} \widehat{\mho}_{c}\right\rangle\right\},
\end{aligned}
$$

where $N$ is normalization coefficient, and we have $|N|^{2}=$ $\left[2+\left\langle\psi_{a b} \mid \psi_{a c}\right\rangle\right]^{-1}$. Accordingly, one can obtain the energy value of the $\mho_{a}$

$$
\begin{aligned}
E_{a}\left(\lambda, \vec{r}_{b c}\right)= & \left\langle\psi_{a}\left(\vec{r}_{a b}, \vec{r}_{a c}\right)\left|H_{a}\right| \psi_{a}\left(\vec{r}_{a b}, \vec{r}_{a c}\right)\right\rangle \\
= & \frac{1}{1+1 / 2\left\langle\psi_{a b} \mid \psi_{a c}\right\rangle}\left\{E_{2}+\frac{1}{4}\left\langle\psi_{a b}\left|T_{a}\right| \psi_{a c}\right\rangle\right. \\
& +\frac{1}{4}\left\langle\psi_{a c}\left|T_{a}\right| \psi_{a b}\right\rangle+\frac{1}{2}\left\langle\psi_{a b}\left|V_{a b}\right| \psi_{a c}\right\rangle \\
& \left.+\frac{1}{2}\left\langle\psi_{a c}\left|V_{a b}\right| \psi_{a b}\right\rangle\right\},
\end{aligned}
$$

where in the second step, we have used Equation (5) and the symmetry between $b$ and $c$. The $\lambda$ is a scaling parameter which can be determined by the experimental data for the two-body binding energy $E_{2}$. Since the interactions should be relevant to the scaling, both the wave functions and the binding energy should depend on the scaling parameter $\lambda$. Thus, $E_{a}$ is also relevant to the $\lambda$. In general, the values of the $E_{2}$ and $E_{a}$ depend on the isospin state of the threefermion system, which will be illuminated in Section 3.1. The calculation of the $E_{a}$ contains the cross integrals $\left\langle\psi_{a b}\right|$ $\left.\psi_{a c}\right\rangle,\left\langle\psi_{a b}\left|T_{a}\right| \psi_{a c}\right\rangle,\left\langle\psi_{a c}\left|T_{a}\right| \psi_{a b},\left\langle\psi_{a b}\left|V_{a b}\right| \psi_{a c}\right\rangle\right.$, and $\left\langle\psi_{a c}\right|$ $V_{a b}\left|\psi_{a b}\right\rangle$, which can be performed in the ellipsoidal coordi- nate. The calculation details of the cross integrals are presented in Appendix D.

As shown in Figure 1, when the $r_{b c}=0$, the energy value of the fermion $\mho_{a}$ reaches to its minimum. It corresponds to the limit that the $\mho_{b}$ and $\mho_{c}$ are on top of each other; then, the system is reduced to the $\mho_{b} \mho_{c}-\mho_{a}$ quasi-two-body system. If we put the fermion $\mho_{b}$ infinitely far away from the fermion $\mho_{c}$, which corresponds the limit $r_{b c} \longrightarrow \infty$, then the energy $E_{a}$ will tend to the two-body energy eigenvalue $E_{2}$. It can be easily seen from Equation (8), where the overlap integration $\left\langle\psi_{a b} \mid \psi_{a c}\right\rangle,\left\langle\psi_{a b}\left|T_{a}\right| \psi_{a c}\right\rangle,\left\langle\psi_{a c}\left|T_{a}\right| \psi_{a b}\right\rangle,\left\langle\psi_{a b}\right| V_{a b}$ $\left|\psi_{a c}\right\rangle$, and $\left\langle\psi_{a c}\left|V_{a b}\right| \psi_{a b}\right\rangle$ tend to 0 . It corresponds to the case of a two-body system plus a free fermion. If the attraction provided by the one-boson exchange is strong enough, the fermion $\mho_{a}$ can form a two-body bound state with either the $\mho_{b}$ or $\mho_{c}$. In fact, it is the breakup state for the trifermion system. In general, the $E_{2}$ is a number quantity irrelevant to the $r_{b c}$, which is trivial for the trifermion system. Thus, we define the $\mathrm{BO}$ potential as

$$
V_{\mathrm{BO}}\left(\lambda, \vec{r}_{b c}\right)=E_{a}\left(\lambda, \vec{r}_{b c}\right)-E_{a}(\lambda, \infty) \text {. }
$$

Therefore, the physical meaning of the $\mathrm{BO}$ potential between the $\mho_{b}$ and $\mho_{c}$ is the energy eigenvalue of the fermion $\mho_{a}$ relative to that of the breakup state.

2.2. The Configurations of Tritonlike Systems. Similar with the discussions in Ref. [88], we assume the fermions $\mho_{b}$ and $\mho_{c}$ are much heavier than the $\mho_{a}$; then, we can use the $\mathrm{BO}$ approximation to separate the motion of the fermion $\mho_{a}$ from the three-body system. The BO approximation is a kind of adiabatic approximation that we divide the degrees of freedom into a heavy one and a light one. The heavy degree of freedom is the relative motion between the $\mho_{b}$ and $\mho_{c}$. The light degree of freedom is the motion of the fermion $\mho_{a}$ relative to the three-body center of mass. The BO potential can reflect the influence of one fermion on the dynamics of the other two. Then, one can simplify the three-body system into a two-body system $\mho_{b} \mho_{c}$ with the BO potential created by the fermion $\mho_{a}$. The light degree of freedom can be described by the wave function of the $\mho_{a}$ derived in Equation (7). The heavy degree of freedom can be described by a wave function $\Phi\left(\vec{r}_{b c}\right)$ which should be determined by the three-body Schrödinger equation. Then, in this case, the total wave function of the system has the form $\Phi\left(\vec{r}_{b c}\right) \psi\left(\vec{r}_{a b}, \vec{r}_{a c}\right)$.

For the system $\mho_{a} \mho_{b} \mho_{c}$, the application of the BO approximation is not straightforward. However, we can use the BOP method where the $\mathrm{BO}$ potential is still employed to describe the contribution of one of the particles on the dynamics of the other two. Based on the BOP method, one should count the influence of each fermion on the dynamics of the other two fermions one by one for the system $\mho_{a} \mho_{b} \mho_{c}$ . The system has three basic simplification schemes. One is that we divide the system into the two-body subsystem $\mho_{b}$ $\mho_{c}$ with the BO potential created by the fermion $\mho_{a}$. One is $\mho_{c} \mho_{a}$ with the BO potential created by the fermion $\mho_{b}$. The other one is $\mho_{a} \mho_{b}$ with the $\mathrm{BO}$ potential created by 
the fermion $\mho_{c}$. The three different simplification schemes lead to three different configurations. For simplicity, we use $\psi_{a}=\Phi\left(\vec{r}_{b c}\right) \psi\left(\vec{r}_{a b}, \vec{r}_{a c}\right), \psi_{b}=\Phi\left(\vec{r}_{a c}\right) \psi\left(\vec{r}_{a b}, \vec{r}_{b c}\right)$, and $\psi_{c}=\Phi\left(\vec{r}_{a b}\right) \psi\left(\vec{r}_{b c}, \vec{r}_{a c}\right)$ to represent the three basic configuration wave functions, where $\Phi\left(\vec{r}_{b c}\right), \Phi\left(\vec{r}_{a c}\right)$, and $\Phi\left(\vec{r}_{a b}\right)$ are undetermined functions that need to be solved. The $\psi($ $\left.\vec{r}_{a b}, \vec{r}_{b c}\right)$ and $\psi\left(\vec{r}_{b c}, \vec{r}_{a c}\right)$ can be obtained by the similar procedures for the $\psi\left(\vec{r}_{a b}, \vec{r}_{a c}\right)$. The configuration wave function $\psi_{a}$ denotes the scheme that we omit the fermion $\mho_{a}$ and add the BO potential provided by the fermion $\mho_{a}$ instead. The $\psi_{b}$ and $\psi_{c}$ denote the $\mathrm{BO}$ potential provided by the fermion $\mho_{b}$ and $\mho_{c}$, respectively.

The three configuration functions can be regarded as a set of basis states and constitute a configuration space $\left\{\psi_{a}\right.$, $\left.\psi_{b}, \psi_{c}\right\}$. We expect that a common approximation for the three-body eigenstate can be expressed as a superposition of the three kinds of basic configurations. Thus, the interpolating wave function of the three-body wave function can be written as

$$
\Psi_{T}=\alpha \psi_{a}+\beta \psi_{b}+\gamma \psi_{c}=\left(\begin{array}{l}
\alpha \\
\beta \\
\gamma
\end{array}\right),
$$

where the $\alpha, \beta$, and $\gamma$ are the expansion coefficients. The three basic configuration functions in the channel space \{ $\left.\widehat{\mho}_{a} \mho_{b} \mho_{c}, \mho_{a} \widehat{\mho}_{b} \mho_{c}, \mho_{a} \mho_{b} \widehat{\mho}_{c}\right\}$ according to Equation (7) read

$$
\begin{aligned}
& \psi_{a}=N \Phi\left(\vec{r}_{b c}\right)\left(\begin{array}{c}
\frac{1}{\sqrt{2}}\left[\psi\left(\vec{r}_{a b}\right)+\psi\left(\vec{r}_{a c}\right)\right] \\
\frac{1}{\sqrt{2}} \psi\left(\vec{r}_{a b}\right) \\
\frac{1}{\sqrt{2}} \psi\left(\vec{r}_{a c}\right)
\end{array}\right) \text {, } \\
& \psi_{b}=N \Phi\left(\vec{r}_{a c}\right)\left(\begin{array}{c}
\frac{1}{\sqrt{2}} \psi\left(\vec{r}_{a b}\right) \\
\frac{1}{\sqrt{2}}\left[\psi\left(\vec{r}_{a b}\right)+\psi\left(\vec{r}_{b c}\right)\right] \\
\frac{1}{\sqrt{2}} \psi\left(\vec{r}_{b c}\right)
\end{array}\right) \text {, } \\
& \psi_{c}=N \Phi\left(\vec{r}_{a b}\right)\left(\begin{array}{c}
\frac{1}{\sqrt{2}} \psi\left(\vec{r}_{a c}\right) \\
\frac{1}{\sqrt{2}} \psi\left(\vec{r}_{b c}\right) \\
\frac{1}{\sqrt{2}}\left[\psi\left(\vec{r}_{b c}\right)+\psi\left(\vec{r}_{a c}\right)\right]
\end{array}\right) \text {, }
\end{aligned}
$$

where the $\Phi\left(\vec{r}_{b c}\right)$ can be expanded as a set of Laguerre polynomials $\Phi\left(\vec{r}_{b c}\right)=\sum_{i} a_{i} \phi_{i}\left(\vec{r}_{b c}\right) . N$ is a normalization con- stant. We define the $i$ th order of the configuration functions as $\psi_{a}^{i}=\phi_{i}\left(\vec{r}_{b c}\right) \psi\left(\vec{r}_{a b}, \vec{r}_{a c}\right), \psi_{b}^{i}=\phi_{i}\left(\vec{r}_{a c}\right) \psi\left(\vec{r}_{a b}\right.$, $\left.\vec{r}_{b c}\right)$, and $\psi_{c}^{i}=\phi_{i}\left(\vec{r}_{a b}\right) \psi\left(\vec{r}_{b c}, \vec{r}_{a c}\right)$.

Then, the configuration space $\left\{\psi_{a}, \psi_{b}, \psi_{c}\right\}$ can be expanded as $\left\{\psi_{a}^{i}, \cdots, \psi_{b}^{i}, \cdots, \psi_{c}^{i}\right\}$. In order to get an orthogonal basis, we should orthonormalize the $\left\{\psi_{a}^{i}, \cdots, \psi_{b}^{i}, \cdots, \psi_{c}^{i}\right\}$ into a new basis $\left\{\tilde{\psi}_{a}^{i}, \cdots, \tilde{\psi}_{b}^{i}, \cdots, \tilde{\psi}_{c}^{i}\right\}$. The $\tilde{\psi}_{a}^{i}, \tilde{\psi}_{b}^{i}$, and $\tilde{\psi}_{c}^{i}$ are used to denote the $i$ th order of the new configuration functions $\tilde{\psi}_{a}$, $\tilde{\psi}_{b}$, and $\tilde{\psi}_{c}$, respectively. Then, the interpolating wave function of the three-body system can be written as

$$
\left(\begin{array}{c}
\tilde{\psi}_{a}^{i} \\
\vdots \\
\tilde{\psi}_{b}^{i} \\
\vdots \\
\tilde{\psi}_{c}^{i}
\end{array}\right)=\left(\begin{array}{c}
\frac{1}{N_{i}}\left[\left(\psi_{a}^{i}+\psi_{b}^{i}+\psi_{c}^{i}\right)-\sum_{i} x_{i j} \psi_{a}^{j}\right] \\
\vdots \\
\frac{1}{N_{i}}\left[\left(\psi_{a}^{i}+\psi_{b}^{i}+\psi_{c}^{i}\right)-\sum_{i} x_{i j} \psi_{b}^{j}\right] \\
\vdots \\
\frac{1}{N_{i}}\left[\left(\psi_{a}^{i}+\psi_{b}^{i}+\psi_{c}^{i}\right)-\sum_{i} x_{i j} \psi_{c}^{j}\right]
\end{array}\right),
$$

where the $x_{i j}$ and $N_{i}$ are the parameter matrix and the normalization coefficient, respectively, which need to be determined later. Because of the interchange symmetry for the $\mho \mho \mho$ system, the parameter matrix $x_{i j}$ in the three configuration functions is the same.

In Equation (12), the ith order of configuration function $\tilde{\psi}_{a}^{i}$ should be orthogonal with the any order of the other configuration function $\tilde{\psi}_{a}^{j}$. Thus, we get the orthonormalization condition

$$
\begin{aligned}
\left\langle\tilde{\psi}_{a}^{i} \mid \tilde{\psi}_{b}^{j}\right\rangle= & \left\langle\frac{1}{N_{i}}\left[\left(\psi_{a}^{i}+\psi_{b}^{i}+\psi_{c}^{i}\right)-\sum_{i} x_{i k} \psi_{a}^{k}\right]\right. \\
& \cdot\left|\frac{1}{N_{j}}\left[\left(\psi_{a}^{j}+\psi_{b}^{j}+\psi_{c}^{j}\right)-\sum_{i} x_{j l} \psi_{b}^{l}\right]\right\rangle=\delta_{i j},
\end{aligned}
$$

which yields

$$
\begin{aligned}
& x_{i k}\left\langle\psi_{a}^{k} \mid \psi_{b}^{l}\right\rangle x_{l j}-x_{i k}\left(\delta_{k j}+2\left\langle\psi_{a}^{k} \mid \psi_{b}^{j}\right\rangle\right)-x_{j l}\left(\delta_{i l}+2\left\langle\psi_{a}^{i} \mid \psi_{b}^{l}\right\rangle\right) \\
& \quad+3 \delta_{i j}+6\left\langle\psi_{a}^{i} \mid \psi_{b}^{j}\right\rangle=0 .
\end{aligned}
$$

It will determine the parameter matrix $x_{i j}$. The orthomoraliztion condition also gives

$$
\frac{1}{N_{i}^{*} N_{j}}\left[3 \delta_{i j}+6\left\langle\psi_{a}^{i} \mid \psi_{b}^{j}\right\rangle-2 x_{i j}-4 \sum_{m} x_{i m}\left\langle\psi_{a}^{m} \mid \psi_{b}^{j}\right\rangle+\sum_{n} x_{\mathrm{in}} x_{n j}\right]=\delta_{i j} .
$$


It will determine the normalization coefficient $N_{i}$. We get an orthonormalized configuration basis after solving the equations for the $x_{i j}$ and $N_{i}$. Then, the eigenvector for the three-body system $\mho \mho \mho$ can be written as a vector in the new configuration space $\left\{\tilde{\psi}_{a}, \tilde{\psi}_{b}, \tilde{\psi}_{c}\right\}$. Thus, the eigenvector for the three-body system has the form

$$
\tilde{\Psi}_{T}=\sum_{i} \tilde{\alpha}_{i} \tilde{\psi}_{a}^{i}+\sum_{j} \tilde{\beta}_{j} \tilde{\psi}_{b}^{j}+\sum_{k} \tilde{\gamma}_{k} \tilde{\psi}_{c}^{k}=\left(\begin{array}{c}
\tilde{\alpha}_{i} \\
\vdots \\
\tilde{\beta}_{i} \\
\vdots \\
\tilde{\gamma}_{i}
\end{array}\right),
$$

where the $\tilde{\alpha}_{i}, \tilde{\beta}_{i}$, and $\tilde{\gamma}_{i}$ are the $i$ th order expansion coefficients of the new configuration basis.

2.3. Three-Body Schrödinger Equation and Its Corrections. We define a reduced Hamiltonian as

$$
\mathscr{H}=H-E_{2} .
$$

Without the kinetic energy of the center of mass for the three-body system, the explicit Hamiltonian has the form

$$
H_{T}=\left[\begin{array}{ccc}
T_{*}+T^{\prime} & V\left(\vec{r}_{a b}\right) & V\left(\vec{r}_{a c}\right) \\
V\left(\vec{r}_{a b}\right) & T_{*}+T_{*}^{\prime} & V\left(\vec{r}_{b c}\right) \\
V\left(\vec{r}_{a c}\right) & V\left(\vec{r}_{b c}\right) & T_{*}+T_{*}^{\prime}
\end{array}\right],
$$

where $V=V_{\pi}+V_{\eta}+V_{\rho}+V_{\omega}+V_{\phi}+V_{\sigma}$. We have used the relation $T_{a}+T_{b}+T_{c}=T_{*}+T^{\prime}{ }_{*}+T^{\prime}{ }_{C} \cdot T^{\prime}{ }_{C}$ is the kinetic energy of the center of mass for the threebody system. $T_{*}=-(1 / 2 \mu) \nabla_{a b}^{2}$ and $T^{\prime}{ }_{*}=-\left(1 / 2 \mu^{\prime}\right) \nabla_{\xi}^{2}$ are the kinetic energy operators, and the corresponding reduced masses are $\mu=M / 2$ and $\mu^{\prime}=2 / 3 M$. Here, and $\nabla_{\xi}^{2}=(1 / \xi)\left(d^{2} / d \xi^{2}\right) \xi-\left(\vec{L}_{\xi}^{2} / \xi^{2}\right)$ with $\vec{\xi}=\vec{r}_{a b} / 2-\vec{r}_{b c}$, where $\vec{r}_{b c}$ is the direction of the fermion $\mho_{b}$ relative to the fermion $\mho_{c} \cdot \vec{L}_{\xi}$ is the relative angular momentum operator between two-body center of mass for the fermions $\mho_{a}$ and $\mho_{b}$ and the fermion $\mho_{c} \cdot \vec{L}_{a b}$ is the angular momentum operator between the fermions $\mho_{a}$ and $\mho_{b}$.

We can rewrite the total Hamiltonian for the three-body system in the configuration space $\left\{\tilde{\psi}_{a}^{i}, \cdots, \tilde{\psi}_{b}^{i}, \cdots, \tilde{\psi}_{c}^{i}\right\}$ as

$$
\begin{aligned}
H_{T}= & \left(\begin{array}{ccccc}
H_{a a}^{i j} & \cdots & H_{a b}^{i j} & \cdots & H_{a c}^{i j} \\
\vdots & \ddots & \vdots & \ddots & \vdots \\
H_{b a}^{i j} & \cdots & H_{b b}^{i j} & \cdots & H_{b c}^{i j} \\
\vdots & \ddots & \vdots & \ddots & \vdots \\
H_{c a}^{i j} & \cdots & H_{c b}^{i j} & \cdots & H_{c c}^{i j}
\end{array}\right) \\
= & \left(\begin{array}{ccccc}
H_{a a}^{i j} & \cdots & H_{a b}^{i j} & \cdots & H_{a c}^{i j} \\
\vdots & \ddots & \vdots & \ddots & \vdots \\
H_{b a}^{i j} & \cdots & H_{b b}^{i j} & \cdots & H_{b c}^{i j} \\
\vdots & \ddots & \vdots & \ddots & \vdots \\
H_{c a}^{i j} & \cdots & H_{c b}^{i j} & \cdots & H_{c c}^{i j}
\end{array}\right) \\
& +E_{2}\left(\begin{array}{ccccc}
1 & \cdots & 0 & \cdots & 0 \\
\vdots & \ddots & \vdots & . & \vdots \\
0 & \cdots & 1 & \cdots & 0 \\
\vdots & \ddots & \vdots & \ddots & \vdots \\
0 & \cdots & 0 & \cdots & 1
\end{array}\right),
\end{aligned}
$$

with $H_{m n}=\left\langle\tilde{\psi}_{m}|H| \tilde{\psi}_{m}\right\rangle(m, n=a, b, c)$.

The total reduced Hamiltonian for the three-body system $\mho \mho \mho$ in the configuration space $\left\{\tilde{\psi}_{a}^{i}, \cdots, \tilde{\psi}_{b}^{i}, \cdots, \tilde{\psi}_{c}^{i}\right\}$ reads

$$
\mathscr{H}_{T}=\left(\begin{array}{ccccc}
H_{a a} & \cdots & H_{a b} & \cdots & H_{a c} \\
\vdots & \ddots & \vdots & \ddots & \vdots \\
H_{b a} & \cdots & H_{b b} & \cdots & H_{b c} \\
\vdots & \ddots & \vdots & \ddots & \vdots \\
H_{c a} & \cdots & H_{c b} & \cdots & H_{c c}
\end{array}\right),
$$

with $\mathscr{H}_{m n}=\left\langle\tilde{\psi}_{m}|\mathscr{H}| \tilde{\psi}_{m}\right\rangle(m, n=a, b, c)$. Thus, we have $H_{T}=\mathscr{H}_{T}+E_{2}$. The explicit form of the matrix element $\mathscr{H}_{a a}$ is given in Appendix A. Given the above definitions, the three-body Schrödinger equation $\mathscr{H}_{T} \Psi_{T}=E_{3} \Psi_{T}$ reads

$$
\left(\begin{array}{ccccc}
H_{a a} & \cdots & H_{a b} & \cdots & H_{a c} \\
\vdots & \ddots & \vdots & . & \vdots \\
H_{b a} & \cdots & H_{b b} & \cdots & H_{b c} \\
\vdots & . & \vdots & \ddots & \vdots \\
H_{c a} & \cdots & H_{c b} & \cdots & H_{c c}
\end{array}\right)\left(\begin{array}{c}
\tilde{\alpha}_{i} \\
\vdots \\
\tilde{\beta}_{i} \\
\vdots \\
\tilde{\gamma}_{i}
\end{array}\right)=E_{3}\left(\begin{array}{c}
\tilde{\alpha}_{i} \\
\vdots \\
\tilde{\beta}_{i} \\
\vdots \\
\tilde{\gamma}_{i}
\end{array}\right) \text {, }
$$

where the energy eigenvalue $E_{3}$ is the reduced threebody energy eigenvalue relative to the breakup state. The total energy eigenvalue relative to the $\mho \mho \mho$ mass threshold is $E_{T}=E_{3}+E_{2}$. 
One may wonder the wave function $\psi_{a}\left(\vec{r}_{a b}, \vec{r}_{a c}\right)$ we used for calculating the $\mathrm{BO}$ potential is too rough. Through simply superpositions of the two components as Equation (7), we get the interpolating wave functions of the fermion $\mho_{a}$. The $\psi\left(\vec{r}_{a b}\right)$ and $\psi\left(\vec{r}_{a c}\right)$ are simply obtained by solving the two-body Schrödinger equations for the $\mho_{a} \mho_{b}$ and $\mho_{a}$ $\mho_{c}$. In fact, the existence of the $\mho_{c}$ will distort the shape of the wave function for the $\mho_{a} \mho_{b}$. We should consider the distortion created by the $\mho_{c}$ while calculating the BO potential between the $\mho_{a}$ and $\mho_{b}$. After solving the three-body Schrödinger equation as Equation (21), we obtain the distorted wave functions $\Phi\left(\vec{r}_{b c}\right), \Phi\left(\vec{r}_{a b}\right)$, and $\Phi\left(\vec{r}_{a c}\right)$ in Equation (11). Therefore, we should use $\Phi\left(\vec{r}_{a b}\right)$ and $\Phi\left(\vec{r}_{a c}\right)$ to calculate the $\mathrm{BO}$ potential created by the $\mho_{a}$, which can be regarded as the first-order corrections. With the distortion effect, the wave function for the $\mho_{a}$ reads

$$
\begin{aligned}
\psi_{a}^{\prime}\left(r_{a b}, r_{a c}\right)= & N^{\prime}\left\{\left[\frac{1}{\sqrt{2}} \Phi\left(r_{a b}\right)+\frac{1}{\sqrt{2}} \Phi\left(r_{a c}\right)\right]\left|\widehat{\mho}_{a} \mho_{b} \mho_{c}\right\rangle\right. \\
& +\frac{1}{\sqrt{2}} \Phi\left(r_{a b}\right)\left|\mho_{a} \widehat{\mho}_{b} \mho_{c}\right\rangle \\
& \left.+\frac{1}{\sqrt{2}} \Phi\left(r_{a c}\right)\left|\mho_{a} \mho_{b} \widehat{\mho}_{c}\right\rangle\right\},
\end{aligned}
$$

where $N^{\prime}$ is the normalization coefficient, and we have $\left|N^{\prime}\right|^{2}=\left[2+\left\langle\Phi_{a b} \mid \Phi_{a c}\right\rangle\right]^{-1}$. Accordingly, considering the distortion effect, one can obtain the energy value of the $\mho_{a}$

$$
\begin{aligned}
E_{a}^{\prime}\left(\Lambda, \vec{r}_{b c}\right)= & \left\langle\psi_{a}^{\prime}\left(\vec{r}_{a b}, \vec{r}_{a c}\right)\left|H_{a}\right| \psi_{a}^{\prime}\left(\vec{r}_{a b}, \vec{r}_{a c}\right)\right\rangle \\
= & \frac{1}{1+1 / 2\left\langle\Phi_{a b} \mid \Phi_{a c}\right\rangle}\left\{E_{2}+\frac{1}{4}\left\langle\Phi_{a b}\left|T_{a}\right| \Phi_{a c}\right\rangle\right. \\
& +\frac{1}{4}\left\langle\Phi_{a c}\left|T_{a}\right| \Phi_{a b}\right\rangle+\frac{1}{2}\left\langle\Phi_{a b}\left|V_{a b}\right| \Phi_{a c}\right\rangle \\
& \left.+\frac{1}{2}\left\langle\Phi_{a c}\left|V_{a b}\right| \Phi_{a b}\right\rangle\right\}
\end{aligned}
$$
tion as

We define the BO potential with the distortion correc-

$$
V_{B O}^{\prime}\left(\Lambda, \vec{r}_{b c}\right)=E_{a}^{\prime}\left(\Lambda, \vec{r}_{b c}\right)-E_{a}^{\prime}(\Lambda, \infty)
$$

Then, with the similar procedures discussed in Sections 2.2 and 2.3, we can arrive the final three-body Schrödinger equation. The solutions of this three-body Schrödinger equation may unveil the binding information of the threebody system $\mho_{a} \mho_{b} \mho_{c}$.

\section{Applications to the Baryon Octet}

We have constructed the formalism for the system of three identical fermions above. Now we return to the systems mainly concerned in this work, which are the tritonlike sys-

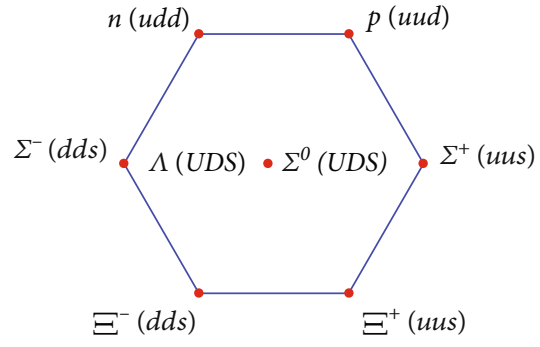

FIgURE 3: Diagram of baryon octet. We have included the quark content.

tems composed of three identical hadrons from the octet of the $1 / 2^{+}$baryon, i.e., $N N N, \Lambda \Lambda \Lambda, \Xi \Xi \Xi$, and $\Sigma \Sigma \Sigma$. We shall perform investigations on the bound states of these tritonlike systems and study the dependence of their three-body binding energies on the two-body binding energies of their twobody subsystems.

3.1. The Dynamics of the Baryon Octet. Under the SU(3)-flavor symmetry, the states $p, n, \Xi^{0}, \Xi^{-}, \Sigma^{+}, \Sigma^{0}, \Sigma^{-}$, and $\Lambda$ are grouped into the ground state baryon octet. We draw the diagram of the baryon octet in Figure 3, where we have included the quark content. Among them, $\Lambda$ is an isoscalar; $\left\{\Xi^{0}, \Xi^{-}\right\}$and $\{p, n\}$ are isospinors; $\left\{\Sigma^{+}, \Sigma^{0}, \Sigma^{-}\right\}$is an isovector. We denote these states by using $\Lambda, \Xi, N$, and $\Sigma$. The wave function of a tritonlike system consists of its isospin, spin, and spatial wave functions. For simplicity, we define the isospin wave function of a tritonlike system as | $\left.I_{2}, I_{3}, I_{3 z}\right\rangle$, where the $I_{2}$ is the isospin of its two-body subsystem and the $I_{3}$ and $I_{3 z}$ denote the total isospin of the whole system and its $z$ direction, respectively. We collect the isospin wave functions of them in Appendix B. Since the strong interactions conserve isospin symmetry, the effective potentials and eigenvalues for a specific system do not depend on the third components of its isospin. It is adequate to take the isospin wave function of a tritonlike system as $\left|I_{2}, I_{3}\right\rangle$ in our calculations.

The total wave function of a system that is made of three identical hadrons of the baryon octet is the product of its isospin, spatial, and spin wave functions,

$$
\Psi_{T} \sim \Psi_{I_{2}, I_{3}, I_{3 z}} \otimes \Psi_{\text {spatial }} \otimes \Psi_{S_{2}, S_{3}, S_{3 z}} .
$$

Since we focus on the systems composed of three identical baryons, the generalized identity principle constricts their isospin and spin wave functions to be antisymmetric. Thus, only some specific combinations of the wave functions can survive. Generally speaking, the two-body force in these tritonlike systems depends on their isospin and spin. However, it cannot be arbitrary due to the constrain from the generalized identity principle. To illuminate it specifically, we use the $\left(I_{2}, S_{2}\right)$ to denote the isospin and spin of the interacting baryons. For the two-body force in the $N N N$ system, only the cases $(1,0)$ and $(0,1)$ survive. For the $\Lambda \Lambda \Lambda$ system, there is only one case $(0,0)$ needs to be considered. For the $\Xi \Xi \Xi$ system, the cases $(1,0)$ and $(0,1)$ should be taken into account. The $\Sigma \Sigma \Sigma$ system only has three cases $(0,0)$, 
$(1,1)$, and $(2,0)$ after considering the generalized identity principle.

We mainly concern the $S$ wave functions in a threebaryon system. It is fully symmetric, so either the isospin wave function or spin wave function should be antisymmetric. Since the strong interactions conserve the isospin and the total spin of a system, we use $\left|I_{2}, I_{3}\right\rangle_{I} \otimes\left|S_{2}, S_{3}\right\rangle_{S}$ denotes the state of a three-body system. For the $N N N$ and $\Xi \Xi \Xi$ systems, the possible states could be $|0,1 / 2\rangle_{I} \otimes|1,1 / 2\rangle_{S}$, $|1,1 / 2\rangle_{I} \otimes|0,1 / 2\rangle_{S}, \quad|0,1 / 2\rangle_{I} \otimes|1,3 / 2\rangle_{S}, \quad$ and $|1,3 / 2\rangle_{I} \otimes$ $|0,1 / 2\rangle_{S}$. For the $\Lambda \Lambda \Lambda$ system, only $|0,0\rangle_{I} \otimes|0,1 / 2\rangle_{S}$ sur- vives. For the $\Sigma \Sigma \Sigma$ system, the possible states could be $|0,1\rangle_{I} \otimes|0,1 / 2\rangle_{S}, \quad|1,(0,1,2)\rangle_{I} \otimes|1,1 / 2(3 / 2)\rangle_{S}, \quad$ and $|2,(1,2,3)\rangle_{I} \otimes|0,1 / 2\rangle_{S}$

The Nijmegen OBE model can provide us a more accurate effective potentials to describe the hyperon-hyperon interactions. Since it contains full channel coupling, the calculations extend to the trihyperon systems will be quite complicated. For simplicity, we use the basic OBE model, i.e., without any channel coupling. Based on the basic OBE model, we build the Lagrangians for $N / \Lambda / \Xi / \Sigma$ with interactions from $\pi / \eta / \rho / \omega$ $/ \phi / \sigma$. The Lagrangians under the SU(3)-flavor symmetry read

$$
\begin{aligned}
& \mathscr{L}_{N N}=g_{\pi N N} \bar{N} i \gamma_{5} \tau N \cdot \pi+g_{\eta N N} \bar{N} i \gamma_{5} N \eta+g_{\rho N N} \bar{N} \gamma_{\mu} \tau N \cdot \rho^{\mu}+\frac{f_{\rho N N}}{2 M_{N}} \bar{N} \sigma_{\mu \nu} \tau N \cdot \partial^{\mu} \rho^{v}+g_{\omega N N} \bar{N} \gamma_{\mu} N \omega^{\mu}+\frac{f_{\omega N N}}{2 M_{N}} \bar{N} \sigma_{\mu \nu} N \partial^{\mu} \omega^{\nu}+g_{\sigma N N} \bar{N} N \sigma \\
& \mathscr{L}_{\Lambda \Lambda}=g_{\pi \Lambda \Lambda} \bar{\Lambda} i \gamma_{5} \tau \Lambda \cdot \pi+g_{\eta \Lambda \Lambda} \bar{\Lambda} i \gamma_{5} \Lambda \eta+g_{\rho \Lambda \Lambda} \bar{\Lambda} \gamma_{\mu} \tau \Lambda \cdot \rho^{\mu}+\frac{f_{\rho \Lambda \Lambda}}{2 M_{\Lambda}} \bar{\Lambda} \sigma_{\mu \nu} \tau \Lambda \cdot \partial^{\mu} \rho^{\nu}+g_{\omega N N} \bar{\Lambda} \gamma_{\mu} \Lambda \omega^{\mu} \\
& +\frac{f_{\omega \Lambda \Lambda}}{2 M_{\Lambda}} \bar{\Lambda} \sigma_{\mu \nu} \Lambda \partial^{\mu} \omega^{v}+g_{\phi N N} \bar{\Lambda} \gamma_{\mu} \Lambda \phi^{\mu}+\frac{f_{\phi \Lambda \Lambda}}{2 M_{\Lambda}} \bar{\Lambda} \sigma_{\mu \nu} \Lambda \partial^{\mu} \phi^{v}+g_{\sigma N N} \bar{\Lambda} \Lambda \sigma,
\end{aligned}
$$

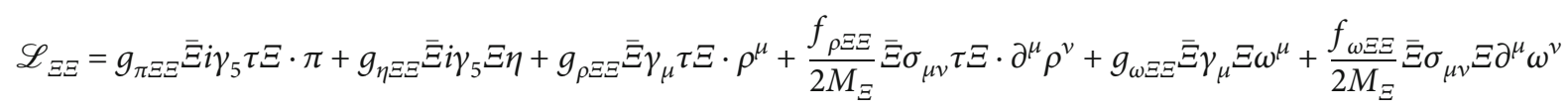

$$
\begin{aligned}
& +g_{\phi \Xi \Xi} \bar{\Xi} \gamma_{\mu} \Xi \phi^{\mu}+\frac{f_{\phi \Xi \Xi}}{2 M_{\Xi}} \bar{\Xi} \sigma_{\mu \nu} \Xi \partial^{\mu} \phi^{\nu}+g_{\sigma N N} \bar{\Xi} \Xi \sigma, \\
& \mathscr{L}_{\Sigma \Sigma}=g_{\pi \Sigma \Sigma}(-i) \bar{\Sigma} i \gamma_{5} \times \Sigma \cdot \pi+g_{\eta \Sigma \Sigma} \bar{\Sigma} \cdot i \gamma_{5} \Sigma \eta+g_{\rho \Sigma \Sigma}(-i) \Sigma \gamma_{\mu} \times \Sigma \cdot \rho^{\mu}+\frac{f_{\rho \Sigma \Sigma}}{2 M_{\Sigma}} \bar{\Sigma} \sigma_{\mu \nu} \times \Sigma \cdot \partial^{\mu} \rho^{\nu}+g_{\omega \Sigma \Sigma} \bar{\Sigma} \gamma_{\mu} \cdot \Sigma \omega^{\mu} \\
& +\frac{f_{\omega \Sigma \Sigma}}{2 M_{\Sigma}} \bar{\Sigma} \sigma_{\mu \nu} \cdot \Sigma \partial^{\mu} \omega^{\nu}+g_{\phi \Sigma \Sigma} \bar{\Sigma} \gamma_{\mu} \cdot \Sigma \phi^{\mu}+\frac{f_{\phi \Sigma \Sigma}}{2 M_{\Sigma}} \bar{\Sigma} \sigma_{\mu \nu} \cdot \Sigma \partial^{\mu} \phi^{\nu}+g_{\sigma \Sigma \Sigma} \bar{\Sigma} \cdot \Sigma \sigma
\end{aligned}
$$

where we have introduced the notations

$$
\begin{gathered}
N=\left(\begin{array}{c}
p \\
n
\end{array}\right), \quad \Xi=\left(\begin{array}{c}
\Xi^{0} \\
\Xi^{-}
\end{array}\right), \\
\Sigma=\left\{\frac{1}{\sqrt{2}}\left(-\Sigma^{+}+\Sigma^{-}\right), \frac{i}{\sqrt{2}}\left(-\Sigma^{+}-\Sigma^{-}\right), \Sigma^{0}\right\}, \Lambda=\Lambda^{0},
\end{gathered}
$$

to represent the corresponding baryon fields. The coefficients $g_{\pi N N}, g_{\pi \Xi \Xi}, g_{\pi \Sigma \Sigma}, f_{\rho N N}$, etc., are the coupling constants. $\tau=\left\{\tau_{1}, \tau_{2}, \tau_{3}\right\}$ are the Pauli matrices, and $\pi=\left\{1 / \sqrt{2}\left(\pi^{+}+\pi^{-}\right), i / \sqrt{2}\left(\pi^{+}-\pi^{-}\right), \pi^{0}\right\}$ are the $\pi$ fields. $\rho$ $=\left\{1 / \sqrt{2}\left(\rho^{+}+\rho^{-}\right), i / \sqrt{2}\left(\rho^{+}-\rho^{-}\right), \rho^{0}\right\}$ are the $\rho$ fields.

The well-known coupling constants for the nucleon in Equation (26) can be extracted from experiment data. For the coupling constants in Equations (27)-(29), we will estimate them by using the nucleon-meson coupling constant as inputs with the help of quark model. The details of the procedure and the specific expression of the nucleonmeson coupling constants at the quark level can be found in Ref. [22]. We list the formulas which relate the coupling constants for the hyperons to coupling constants for the nucleon below.

$\Lambda: g_{\eta \Lambda \Lambda}=-2 g_{\eta N N}\left(M_{\Lambda} / M_{N}\right), \quad g_{\sigma \Lambda \Lambda}=g_{\sigma N N}, g_{\omega \Lambda \Lambda}=2 / 3$ $g_{\omega N N}, f_{\omega \Lambda \Lambda}=-2 / 3 g_{\omega N N}, g_{\phi \Lambda \Lambda}=\sqrt{2} g_{\rho N N}, f_{\phi \Lambda \Lambda}=2 \sqrt{2} g_{\rho N N}$, and $f_{\phi \Lambda \Lambda}=2 \sqrt{2} g_{\rho N N}$.

$\Xi: g_{\pi \Xi \Xi}=-1 / 5 g_{\pi N N}\left(M_{\Xi} / M_{N}\right), g_{\eta \Xi \Xi}=-3 g_{\eta N}\left(M_{\Xi} / M_{N}\right)$, $g_{\sigma \Xi \Xi}=g_{\sigma N N}, \quad g_{\rho \Xi \Xi}=g_{\rho N N}, \quad f_{\rho \Xi \Xi}=-g_{\rho N N}\left(M_{\Xi} / M_{N}+1\right)$, $g_{\omega \Xi \Xi}=1 / 3 g_{\omega N N}, \quad f_{\omega \Xi \Xi}=-1 / 3 g_{\omega N N}\left(M_{\Xi} / M_{N}+1\right), \quad g_{\phi \Xi \Xi}=2$ $\sqrt{2} g_{\rho N N}$, and $f_{\phi \Xi \Xi}=2 \sqrt{2} g_{\rho N N}\left(2\left(M_{\Xi} / M_{N}\right)-1\right)$.

$\Sigma: \quad g_{\pi \Sigma \Sigma}=4 / 5 g_{\pi N N}\left(M_{\Xi} / M_{N}\right), \quad g_{\eta \Sigma \Sigma}=2 g_{\eta N N}\left(M_{\Xi} / M_{N}\right)$, $g_{\sigma \Sigma \Sigma}=g_{\sigma N N}, \quad g_{\rho \Sigma \Sigma}=2 g_{\rho N N}, f_{\rho \Sigma \Sigma}=g_{\rho N N}\left(4\left(M_{\Xi} / M_{N}\right)-1\right)$, $g_{\omega \Sigma \Sigma}=2 / 3 g_{\omega N N}, f_{\omega \Sigma \Sigma}=2 / 3 g_{\omega N N}\left(2\left(M_{\Xi} / M_{N}\right)-1\right), \quad g_{\phi \Sigma \Sigma}=$ $\sqrt{2} g_{\rho N N}$, and $f_{\phi \Sigma \Sigma}=-\sqrt{2} g_{\rho N N}\left(M_{\Xi} / M_{N}+1\right)$.

By fitting to experimental data, we adopt the values $g_{\pi N N}=13.07, g_{\eta N N}=2.24, g_{\sigma N N}=8.46, g_{\rho N N}=3.25, f_{\rho N N}$ $=6.1 g_{\rho N N}, g_{\omega N N}=15.85$, and $f_{\omega N N}=0$ from Refs. [22, $79,89,90]$. We collect the numerical values of them in Table 1. 
TABLE 1: The coupling constants and masses in our calculation. The masses are taken from the PDG [91].

\begin{tabular}{|c|c|c|c|c|c|c|c|}
\hline & Mass $(\mathrm{MeV})$ & $\pi$ & $\eta$ & $\sigma$ & $\rho$ & $\omega$ & $\phi$ \\
\hline Mass $(\mathrm{MeV})$ & & $m_{\pi}=139.00$ & $m_{\eta}=547.85$ & $m_{\sigma}=600.00$ & $m_{\rho}=775.49$ & $m_{\omega}=782.65$ & $m_{\phi}=1019.50$ \\
\hline \multirow[t]{2}{*}{$N$} & $M_{N}=939.00$ & $g_{\pi N N}=13.07$ & $g_{\eta N N}=2.24$ & $g_{\sigma N N}=8.46$ & $g_{\rho N N}=3.25$ & $g_{\omega N N}=15.85$ & \\
\hline & & & & & $f_{\rho N N}=19.83$ & $f_{\omega N N}=0$ & \\
\hline \multirow[t]{2}{*}{$\Lambda$} & $M_{\Lambda}=1115.68$ & $g_{\pi \Lambda \Lambda}=0$ & $g_{\eta \Lambda \Lambda}=-5.33$ & $g_{\sigma \Lambda \Lambda}=8.46$ & $g_{\rho \Lambda \Lambda}=0$ & $g_{\omega \Lambda \Lambda}=10.57$ & $g_{\phi \Lambda \Lambda}=4.60$ \\
\hline & & & & & $f_{\rho \Lambda \Lambda}=0$ & $f_{\omega \Lambda \Lambda}=-10.57$ & $f_{\phi \Lambda \Lambda}=9.19$ \\
\hline \multirow[t]{2}{*}{$\Xi$} & $M_{\Lambda}=1318.28$ & $g_{\pi \Lambda \Lambda}=-3.67$ & $g_{\eta \Lambda \Lambda}=-9.44$ & $g_{\sigma \Lambda \Lambda}=8.46$ & $g_{\rho \Lambda \Lambda}=3.25$ & $g_{\omega \Lambda \Lambda}=5.28$ & $g_{\phi \Lambda \Lambda}=9.05$ \\
\hline & & & & & $f_{\rho \Lambda \Lambda}=-7.81$ & $f_{\omega \Lambda \Lambda}=-12.70$ & $f_{\phi \Lambda \Lambda}=16.36$ \\
\hline \multirow[t]{2}{*}{$\Sigma$} & $M_{\Lambda}=1193.15$ & $g_{\pi \Lambda \Lambda}=13.29$ & $g_{\eta \Lambda \Lambda}=5.70$ & $g_{\sigma \Lambda \Lambda}=8.46$ & $g_{\rho \Lambda \Lambda}=6.50$ & $g_{\omega \Lambda \Lambda}=10.57$ & $g_{\phi \Lambda \Lambda}=4.60$ \\
\hline & & & & & $f_{\rho \Lambda \Lambda}=13.27$ & $f_{\omega \Lambda \Lambda}=16.29$ & $f_{\phi \Lambda \Lambda}=-10.44$ \\
\hline
\end{tabular}

Given the Lagrangians above, we can derive the effective potentials for the two-body interactions of the tritonlike systems. Expanding the $T$ matrices with external momenta to the leading order, one obtains the effective potentials for the two-body interactions. Then, the effective potentials in coordinate space can be derived by Fourier transformation

$$
V(\vec{r})=\frac{1}{(2 \pi)^{3}} \int d^{3} \vec{q} e^{i \vec{q} \cdot \vec{r}} T(\vec{q}) F^{2}(\vec{q})
$$

where $F(\vec{q})$ is the monopole form factor attached to each scattering vertex. The form is

$$
F(q)=\frac{\lambda^{2}-m_{\alpha}^{2}}{\lambda^{2}-q^{2}}=\frac{\lambda^{2}-m_{\alpha}^{2}}{\lambda^{2}+\vec{q}^{2}}
$$

with the mass of exchange boson $m_{\alpha}$. The $\lambda$ is the cutoff parameter which cannot be well determined from fundamental theories due to the nonperturbative effect. It is a rough way to reflect the nonpoint-like hadronic structures and suppress the contribution from UV energies.

In general, the two-body effective potentials for tribaryon system consist of the central force term, the spin-spin interaction term, the spin-orbital interaction term, and the tensor force term. With the definition of the tensor force operator $S_{12}(\widehat{r})=3\left(\vec{\sigma}_{1} \cdot \hat{r}\right)\left(\vec{\sigma}_{2} \cdot \hat{r}\right)-\vec{\sigma}_{1} \cdot \vec{\sigma}_{2}$, the effective potentials can be expressed in the following form:

$$
V(\vec{r})=V_{0}(r)+V_{S}(r) \vec{\sigma}_{1} \cdot \vec{\sigma}_{2}+V_{L}(r) \vec{L} \cdot \vec{S}+V_{T}(r) S_{12}(\widehat{r})
$$

TABle 2: Isospin factors. Here, $I$ and $S$ denote the isospin and the spin of the two-body system.

\begin{tabular}{lcccccccc}
\hline \multirow{2}{*}{$(I, S)$} & \multicolumn{2}{c}{$N N$} & $\Lambda \Lambda$ & \multicolumn{3}{c}{$\Xi \Xi$} & \multicolumn{3}{c}{$\sum \Sigma$} & \\
& $(0,1)$ & $(1,0)$ & $(0,0)$ & $(0,1)$ & $(1,0)$ & $(0,0)$ & $(1,1)$ & $(2,0)$ \\
\hline$C_{\pi}^{h h}$ & -3 & 1 & 1 & -3 & 1 & -2 & -1 & 1 \\
$C_{\eta}^{h h}$ & 1 & 1 & 1 & 1 & 1 & 1 & 1 & 1 \\
$C_{\sigma}^{h h}$ & 1 & 1 & 1 & 1 & 1 & 1 & 1 & 1 \\
$C_{\rho}^{h h}$ & -3 & 1 & 1 & -3 & 1 & -2 & -1 & 1 \\
$C_{\omega}^{h h}$ & 1 & 1 & 1 & 1 & 1 & 1 & 1 & 1 \\
$C_{\phi}^{h h}$ & 1 & 1 & 1 & 1 & 1 & 1 & 1 & 1 \\
\hline
\end{tabular}

The total effective potential contains contributions from the $\pi, \eta, \sigma, \rho, \omega$, and $\phi$ exchanges. After working them out one by one and adding them, we have

$$
\begin{aligned}
V_{t o t}^{h h}(\vec{r})= & C_{\pi}^{h h} V_{\pi}(\vec{r} ; h)+C_{\eta}^{h h} V_{\eta}(\vec{r} ; h)+C_{\sigma}^{h h} V_{\sigma}(\vec{r} ; h) \\
& +C_{\rho}^{h h} V_{\rho}(\vec{r} ; h)+C_{\omega}^{h h} V_{\omega}(\vec{r} ; h)+C_{\phi}^{h h} V_{\phi}(\vec{r} ; h),
\end{aligned}
$$

where $h=N, \Lambda, \Xi, \Sigma . C_{\pi}^{h h}, C_{\eta}^{h h}, C_{\sigma}^{h h}, C_{\rho}^{h h}, C_{\omega}^{h h}$, and $C_{\phi}^{h h}$ are the isospin factors. We summarize these isospin factors in Table 2.

The $V_{\pi}(\vec{r} ; h), V_{\eta}(\vec{r} ; h), V_{\sigma}(\vec{r} ; h), V_{\rho}(\vec{r} ; h), V_{\omega}(\vec{r} ; h)$ , and $V_{\phi}(\vec{r} ; h)$ are the effective potentials from each exchanged meson, which have the forms

$$
V_{\pi}(\vec{r} ; h)=-\frac{1}{4 \pi} \frac{g_{\pi h h}^{2}}{4 M_{h}^{2}}\left[f_{1}\left(m_{\pi}, \lambda, r, \vec{\sigma}_{1} \cdot \vec{\sigma}_{2}\right)+f_{2}\left(m_{\pi}, \lambda, r, S_{12}\right)\right],
$$

$$
V_{\eta}(\vec{r} ; h)=-\frac{1}{4 \pi} \frac{g_{\eta h h}^{2}}{4 M_{h}^{2}}\left[f_{1}\left(m_{\eta}, \lambda, r, \vec{\sigma}_{1} \cdot \vec{\sigma}_{2}\right)+f_{2}\left(m_{\eta}, \lambda, r, S_{12}\right)\right],
$$




$$
\begin{aligned}
V_{\sigma}(\vec{r} ; h)= & -\frac{1}{4 \pi} g_{\sigma h h}^{2}\left[f_{3}\left(m_{\sigma}, \lambda, r\right)+\frac{1}{4 M_{h}^{2}} f_{4}\left(m_{\sigma}, \lambda, r\right)\right. \\
& \left.-\frac{1}{2 M_{h}^{2}} f_{5}\left(m_{\sigma}, \lambda, r, \vec{L} \cdot \vec{S}\right)\right], \\
V_{\rho}(\vec{r} ; h)= & \frac{1}{4 \pi}\left[g_{\rho h h}^{2} f_{3}\left(m_{\rho}, \lambda, r\right)-\frac{4 g_{\rho h h} f_{\rho h h}+f_{\rho h h}^{2}}{8 M_{h}^{2}} f_{4}\left(m_{\rho}, \lambda, r\right)\right. \\
+ & \frac{\left(g_{\rho h h}+f_{\rho h h}\right)^{2}}{2 M_{h}^{2}} f_{1}\left(m_{\rho}, \lambda, r, \vec{\sigma}_{1} \cdot \vec{\sigma}_{2}\right) \\
- & \frac{\left(g_{\rho h h}+f_{\rho h h}\right)^{2}}{4 M_{h}^{2}} f_{2}\left(m_{\rho}, \lambda, r, S_{12}\right) \\
- & \frac{3 g_{\rho h h}^{2}+4 g_{\rho h h} f_{\rho h h}+f_{\rho h h}^{2} f_{5}\left(m_{\rho}, \lambda, r, \vec{L} \cdot \vec{S}\right)}{8 M_{h}^{2}} \\
& -\frac{4 g_{\rho h h}^{2}+f_{\rho h h}^{2}}{8 M_{h}^{2}} f_{6}\left(m_{\rho}, \lambda, r\right) \\
+ & \left.\frac{4 g_{\rho h h}^{2}+f_{\rho h h}^{2}}{4 M_{h}^{2}}\left\{\nabla^{2}, f_{3}\left(m_{\rho}, \lambda, r\right)\right\}\right]
\end{aligned}
$$

$$
\begin{aligned}
V_{\omega}(\vec{r} ; h)= & \frac{1}{4 \pi}\left[g_{\omega h h}^{2} f_{3}\left(m_{\omega}, \lambda, r\right)-\frac{4 g_{\omega h h} f_{\omega h h}+f_{\omega h h}^{2}}{8 M_{h}^{2}} f_{4}\left(m_{\omega}, \lambda, r\right)\right. \\
& +\frac{\left(g_{\omega h h}+f_{\omega h h}\right)^{2}}{2 M_{h}^{2}} f_{1}\left(m_{\omega}, \lambda, r, \vec{\sigma}_{1} \cdot \vec{\sigma}_{2}\right) \\
& -\frac{\left(g_{\omega h h}+f_{\omega h h}\right)^{2}}{4 M_{h}^{2}} f_{2}\left(m_{\omega}, \lambda, r, S_{12}\right) \\
& -\frac{3 g_{\omega h h}^{2}+4 g_{\omega h h} f_{\omega h h}+f_{\omega h h}^{2}}{8 M_{h}^{2}} f_{5}\left(m_{\omega}, \lambda, r, \vec{L} \cdot \vec{S}\right) \\
& -\frac{4 g_{\omega h h}^{2}+f_{\omega h h}^{2}}{8 M_{h}^{2}} f_{6}\left(m_{\omega}, \lambda, r\right) \\
& \left.+\frac{4 g_{\omega h h}^{2}+f_{\omega h h}^{2}}{4 M_{h}^{2}}\left\{\nabla^{2}, f_{3}\left(m_{\omega}, \lambda, r\right)\right\}\right]
\end{aligned}
$$

$$
\begin{aligned}
V_{\phi}(\vec{r} ; h)= & \frac{1}{4 \pi}\left[g_{\phi h h}^{2} f_{3}\left(m_{\phi}, \lambda, r\right)-\frac{4 g_{\phi h h} f_{\phi h h}+f_{\phi h h}^{2}}{8 M_{h}^{2}} f_{4}\left(m_{\phi}, \lambda, r\right)\right. \\
& +\frac{\left(g_{\phi h h}+f_{\phi h h}\right)^{2}}{2 M_{h}^{2}} f_{1}\left(m_{\phi}, \lambda, r, \vec{\sigma}_{1} \cdot \vec{\sigma}_{2}\right) \\
& -\frac{\left(g_{\phi h h}+f_{\phi h h}\right)^{2}}{4 M_{h}^{2}} f_{2}\left(m_{\phi}, \lambda, r, S_{12}\right) \\
& -\frac{3 g_{\phi h h}^{2}+4 g_{\phi h h} f_{\phi h h}+f_{\phi h h}^{2}}{8 M_{h}^{2}} f_{5}\left(m_{\phi}, \lambda, r, \vec{L} \cdot \vec{S}\right) \\
& -\frac{4 g_{\phi h h}^{2}+f_{\phi h h}^{2}}{8 M_{h}^{2}} f_{6}\left(m_{\phi}, \lambda, r\right) \\
& \left.+\frac{4 g_{\phi h h}^{2}+f_{\phi h h}^{2}}{4 M_{h}^{2}}\left\{\nabla^{2}, f_{3}\left(m_{\phi}, \lambda, r\right)\right\}\right]
\end{aligned}
$$

$$
\text { tion } \psi_{a b} \text {. Since the } V_{t o t}^{h h}(\vec{r}) \text { depends on the isospin factors }
$$
$C_{\alpha}^{h h}(\alpha=\pi, \eta, \sigma, \rho, \omega, \phi)$, the two-body eigenenergy $E_{2}$ and the two-body wave function $\psi_{a b}$ depend the specific isospin channel of the two-body system. Due to the tensor force in the effective potential leads to S-D wave mixing, we should consider the $\mathrm{D}$ wave part which may contribute the $S$ wave scattering matrix. Thus, we have

$$
\psi\left(\vec{r}_{a b}\right)=\psi_{S}\left(\vec{r}_{a b}\right)+\psi_{D}\left(\vec{r}_{a b}\right)
$$

where $\psi_{S}\left(\vec{r}_{a b}\right)$ and $\psi_{D}\left(\vec{r}_{a b}\right)$ denote the S wave and D wave parts, respectively. The treatments of the operators $\vec{\sigma}_{1} \cdot \vec{\sigma}_{2}, \vec{L} \cdot \vec{S}$, and $S_{12}(\widehat{r})$ which occur in the functions $f_{1-6}$ are straightforward. The two-body subsystem can form either spin-singlet or spin-triplet. For the spin-singlet, we only focus on the ground state ${ }^{1} S_{0}$; then, we have

$$
\vec{\sigma}_{1} \cdot \vec{\sigma}_{2}=-3 \quad \vec{L} \cdot \vec{S}=0 \quad S_{12}(\widehat{r})=0
$$

while for the spin-triplet, we should take both ${ }^{3} S_{1}$ and ${ }^{3} D_{1}$ into account. Thus, the operators can be written in the following matrix form:

$$
\begin{gathered}
\vec{\sigma}_{1} \cdot \vec{\sigma}_{2} \longrightarrow\left(\begin{array}{cc}
1 & 0 \\
0 & 1
\end{array}\right), \\
\vec{L} \cdot \vec{S} \longrightarrow\left(\begin{array}{cc}
0 & 0 \\
0 & -3
\end{array}\right), \\
S_{12}(\widehat{r}) \longrightarrow\left(\begin{array}{cc}
0 & \sqrt{8} \\
\sqrt{8} & -2
\end{array}\right),
\end{gathered}
$$

which leads to the coupled-channel Schrödinger equations.

Along with the same procedures we discussed for the $B$ $B B^{*}$ system in Ref [88], we use the matrix method to solve the Schrödinger equation. The wave function can be expanded as

$$
\psi(\vec{r})=\sum_{i=0}^{n-1} a_{i} \chi_{i 0}(r) \phi_{S}+\sum_{j=0}^{n-1} b_{j} \chi_{j 2}(r) \phi_{D}
$$

by using Laguerre polynomials

$$
\chi_{n l}(r)=\sqrt{\frac{(2 \xi)^{2 l+3} n !}{\Gamma(2 l+3+n)}} r^{l} e^{-\xi r} L_{n}^{2 l+2}(2 \xi r), n=1,2,3 \cdots
$$


with the normalization condition

$$
\int_{0}^{\infty} \chi_{i m}(r) \chi_{j n}(r) r^{2} d r=\delta_{i j} \delta_{m n}
$$

where $\xi$ is the variation parameter. To calculate the energy value of the particle $a$, we use the simplest combination of $\psi\left(\vec{r}_{a b}\right)$ and $\psi\left(\vec{r}_{a c}\right)$ as an interpolating wave function

$$
\begin{aligned}
\psi_{a}\left(\xi, r_{a b}, r_{a c}\right)= & N\left\{\left[\frac{1}{\sqrt{2}} \psi\left(\xi, r_{a b}\right)+\frac{1}{\sqrt{2}} \psi\left(\xi, r_{a c}\right)\right]\left|\widehat{\mho}_{a} \mho_{b} \mho_{c}\right\rangle\right. \\
& +\frac{1}{\sqrt{2}} \psi\left(\xi, r_{a b}\right)\left|\mho_{a} \widehat{\mho}_{b} \mho_{c}\right\rangle \\
& \left.+\frac{1}{\sqrt{2}} \psi\left(\xi, r_{a c}\right)\left|\mho_{a} \mho_{b} \widehat{\mho}_{c}\right\rangle\right\} .
\end{aligned}
$$

Based on this, we can construct the interpolating wave function for the three-body system as discussed in Sections 2.2 and 2.3. The interpolating wave function is relevant to the variation parameter $\xi$. Then, we can change the value of the $\xi$ to find out the lowest energy of the three-body system.

In order to make clear of the binding properties of a three-body system, we should define some quantities for discussions. One is the total three-body energy eigenvalue relative to the three-free fermion threshold. Another one is the reduced three-body energy eigenvalue relative to the breakup state of the three-free fermion threshold. We need to discuss the minimum of the $\mathrm{BO}$ potential $V_{\mathrm{BO}}(0)$ to represent its strength. To emphasize the size of the system is large enough to keep the hadronic picture, we should define the root-mean-square radius of any two fermions in the system by using $r_{\text {rms }}$. Generally, the colorless interactions between nucleons (hyperons) contain tensor forces. Since tensor forces will lead to S-D wave mixing, the contributions from $\mathrm{D}$ wave should be taken into account. Therefore, we should introduce the probabilities for $\mathrm{S}$ wave and $\mathrm{D}$ wave components in any two fermions in the trifermion system.

3.2. Numerical Results for the NNN. The OBE model is very successful in describing the binding properties of deuteron. One may believe that it also describe the dynamics of triton well. Now we apply the BOP method and OBE model to the three-nucleon system. Since the experiment accumulates sufficient data on the system, the application on them can illustrate the feasibility of our formalism. There are two bound states of the three-nucleon system which have been observed in experiment. One is triton, and the other one is helium-3 nucleus. They have the same binding energy and structure without the isospin breaking effect. For simplicity, we investigate the bound state of the NNN system under the isospin symmetry. We list the isospin wave functions of the three-nucleon system in Appendix B.

Applying the Lagrangians in Equation (26) for nucleon, one gets the $T$ matrix for the nucleon-nucleon scattering via the one-boson exchange model. After the Fourier trans- formations as Equation (31), one obtains the effective potentials of the two-body force for the nucleon-nucleon system in coordinate space. The effective potentials contain the contributions from the long-range $\pi / \eta$ exchange, the mediumrange $\sigma$ exchange, and the short-range $\rho / \omega$ exchange. In general, the effective potentials derived from nucleonnucleon scattering consist of the central term, the spin-spin force term, the spin-orbit force term, and the tensor force term. Since the tensor force term leads to the S-D wave mixing, the contribution from $\mathrm{D}$ wave should be taken into account during the computations of the bound state for the NNN system.

In our calculations, there is only one free parameter $\lambda$ in the monopole form factor introduced to reflect the inner structure of the interacting hadrons. It is still quite hard to determine the value of $\lambda$ from fundamental theories. However, we can fix it by using the binding energy of deuteron which is $2.23 \mathrm{MeV}$ from experimental data. When the twobody isospin $I_{2}=0$ and the parameter $\lambda=811.80 \mathrm{MeV}$, we reproduce the binding energy of deuteron $-E_{2}=2.23 \mathrm{MeV}$. In this case, we plot the effective potentials of the isospinsinglet force for the nucleon-nucleon system in Figure 4(a). In order to make a rough estimation of the specific roles of the exchanged bosons in the effective potential, we also plot the $S$ wave effective potentials of each of them in the figure. As shown in Figure 4(a), the $\pi$ exchange provides repulsive force in the short range but shallow attractive force in the medium range. The $\eta$ and $\sigma$ exchange create shallow and deep attraction, respectively. The $\rho$ exchange provides attractive force while the $\omega$ exchange provides repulsive force. Thus, the total effective potential is repulsive in the short range while attractive in the medium range.

Based on the formalism we have built in Section 2, we calculate the NNN system for all of cases of isospin configurations with the parameter $\lambda=811.80 \mathrm{MeV}$. We only find a bound state for the case $|0,1 / 2\rangle_{I} \otimes|1,1 / 2\rangle_{S}$, where the total isospin $I_{3}=1 / 2$ and the isospin of the two-body subsystem $I_{2}=0$ and the total spin $S_{3}=1 / 2$ and the spin of the twobody subsystem $S_{2}=0$. In order to illustrate the contribution of one of the nucleons on the dynamics of the other two, we plot the BO potentials for the state $|0,1 / 2\rangle_{I} \otimes|1,1 / 2\rangle_{S}$ in Figure 4(b). The blue-dotted and red-solid curves are the BO potentials before and after distortion correction, respectively. Both curves have a deep around $0.4 \mathrm{fm}$ as shown in the figure. The correction on the $\mathrm{BO}$ potential makes it become weak. When the distance is larger than a certain value, the $\mathrm{BO}$ potential equals to zero. It corresponds to the breakup state for the NNN system, which consists of a deuteron and a free nucleon.

Through the BOP method with the careful treatment on the $\mathrm{S}-\mathrm{D}$ wave mixing, one can get the dependence of the binding properties on the parameter $\lambda$ as shown in Table 3. There is a three-body bound state with total threebody binding energy in the range of $1.55-30.17 \mathrm{MeV}$, when the parameter $\lambda$ varies from $780 \mathrm{MeV}$ to $920 \mathrm{MeV}$. The corresponding binding energy of its two-body subsystem changes from $0.18 \mathrm{MeV}$ to $9.37 \mathrm{MeV}$. Within the range of the $\lambda$, the reduced three-body binding energy increases from 1.37 MeV to $20.80 \mathrm{MeV}$. The root-mean-square radius of the 


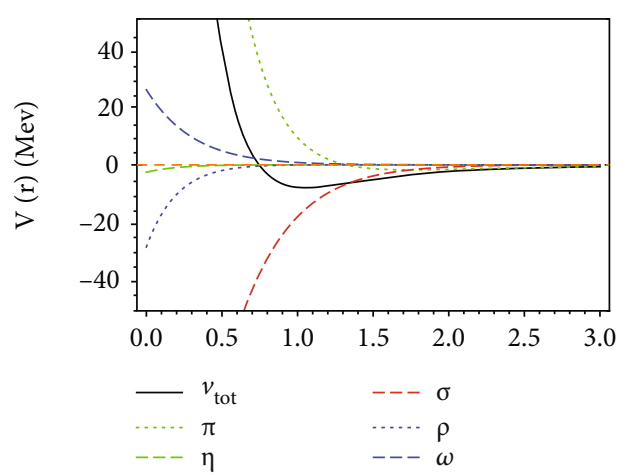

(a)

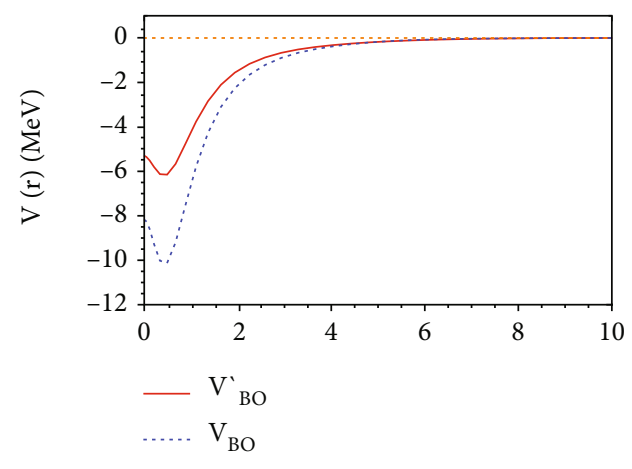

(b)

FIgURE 4: (a) The $S$ wave effective potentials of the isospin-singlet force for the nucleon-nucleon system with the comparison of the contributions of each exchanged boson. (b) The BO potentials for the $N N N$ in the state $|0,1 / 2\rangle_{I} \otimes|1,1 / 2\rangle_{S}$. The dotted and solid lines are the $\mathrm{BO}$ potentials before and after distortion correction, respectively.

TABLE 3: Bound state solutions of the $N N N$ system for the case $|0,1 / 2\rangle_{I} \otimes|1,1 / 2\rangle_{S} . E_{2}$ is the energy eigenvalue of its subsystem. $E_{3}$ is the reduced three-body energy eigenvalue relative to the breakup state of the $N N N$ system. $E_{T}$ is the total three-body energy eigenvalue relative to the $N N N$ threshold. $V_{\mathrm{BO}}(0)$ is the minimum of the $\mathrm{BO}$ potential. $r_{\text {rms }}$ represents the root-mean-square radius of any two $N$ in the $N N N$ system. The $\mathrm{S}$ wave and $\mathrm{D}$ wave represent the probabilities for $\mathrm{S}$ wave and $\mathrm{D}$ wave components in any two $N$ in the NNN system.

\begin{tabular}{lccccccc}
\hline$\Lambda(\mathrm{MeV})$ & $E_{2}(\mathrm{MeV})$ & $E_{3}(\mathrm{MeV})$ & $E_{T}(\mathrm{MeV})$ & $V_{\mathrm{BO}}(0)(\mathrm{MeV})$ & $\mathrm{S}$ wave $(\%)$ & $\mathrm{D}$ wave $(\%)$ & $r_{\mathrm{rms}}(\mathrm{fm})$ \\
\hline .00 & -0.18 & -1.37 & -1.55 & -3.27 & 97.51 & 2.49 & 4.55 \\
.00 & -1.42 & -3.62 & -5.05 & -4.97 & 96.78 & 3.22 \\
.80 & -2.23 & -5.25 & -7.49 & -6.15 & 96.32 & 3.68 \\
.00 & -2.82 & -6.48 & -9.30 & -7.00 & 96.00 & 4.00 & 3.81 \\
.00 & -5.70 & -12.76 & -18.46 & -11.17 & 94.69 & 5.31 & 2.62 \\
.00 & -7.04 & -15.71 & -22.75 & -12.98 & 94.19 & 5.81 \\
.00 & -8.26 & -18.40 & -26.66 & -14.56 & 93.77 & 6.23 \\
.00 & -9.37 & -20.80 & -30.17 & -15.94 & 93.41 & 6.59 & 2.52 \\
\hline
\end{tabular}

system decreases from $4.55 \mathrm{fm}$ to $2.41 \mathrm{fm}$ when the parameter $\lambda$ grows. The three-body bound state of the NNN system is a mixture of the $\mathrm{S}$ and $\mathrm{D}$ wave due to the tensor force in the effective potentials. The proportion of the $S$ wave state is more than $93 \%$. If we shut down the $\mathrm{D}$ wave, we cannot find the binding solution, which means that the S-D wave mixing is very crucial in the formation of the three-body bound state. If we fix the parameter $\lambda=811.80 \mathrm{MeV}$ by reproducing the binding energy of deuteron, the reduced three-body binding energy and total three-body binding energy are $5.25 \mathrm{MeV}$ and $7.49 \mathrm{MeV}$, respectively. As we know, the empirical binding energies of the triton and helium-3 nucleus are $8.48 \mathrm{MeV}$ and $7.80 \mathrm{MeV}$, respectively. Our numerical result $7.49 \mathrm{MeV}$ is comparable with the empirical binding energies. Since we neglect the isospin breaking, there is no numerical difference between the binding energies of the triton and helium-3 nucleus in our computation.

To verify the binding solution is a bound state, we plot the wave functions for any two constituents in the NNN in Figure 5(a). For comparison, we also plot the wave functions for its subsystem $N N$. As shown in the figure, the bound state is dominated by the $S$ wave state with the proportion $96.32 \%$ if the parameter $\Lambda=811.80 \mathrm{MeV}$. The numerical results show that the total three-body binding energy of the NNN system grows as the two-body binding energy of its subsystem $N N$ increases. To show it explicitly, we plot the dependence in Figure 5(b), where $E_{I=1 / 2}^{N N N}$ and $E_{I=0}^{N N}$ denote the total three-body binding energy and two-body binding energy, respectively. One may wonder whether there is a critical value of $E_{I=0}^{N N}$, below which the system $N N N$ has no three-body bound state. In fact, it turns out no such critical value for the system in the state $|0,1 / 2\rangle_{I} \otimes|1,1 / 2\rangle_{S}$. When the two-body binding energy approaches $0 \mathrm{MeV}$, there is still a tiny value $1.14 \mathrm{MeV}$ of the three-body binding energy for the NNN system. No matter how small the two-body binding energy of the subsystem is, the whole system always has a shallow bound state. It is a reminiscent of a Borromean state, in which a three-body system may form a three-body bound state despite none of its subsystem has a bound state. However, since the three-body binding energy is $1.14 \mathrm{MeV}$ and the binding energy of the two-body subsystem approaches $0 \mathrm{MeV}$, it is not a strict Borromean state. It is a critical state between the Borromean state and the ordinary state of the system. For simplicity, we call it the Critical Borromean state. There are two red points in Figure 5(b), where the left one indicates the Critical Borromean state of the system. The right one is the numerical result of triton or helium-3 nucleus. It is a little below the experimental value. Since in 


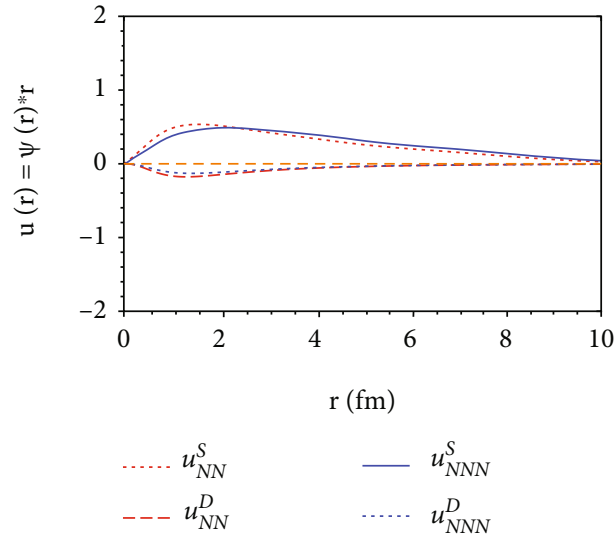

(a)

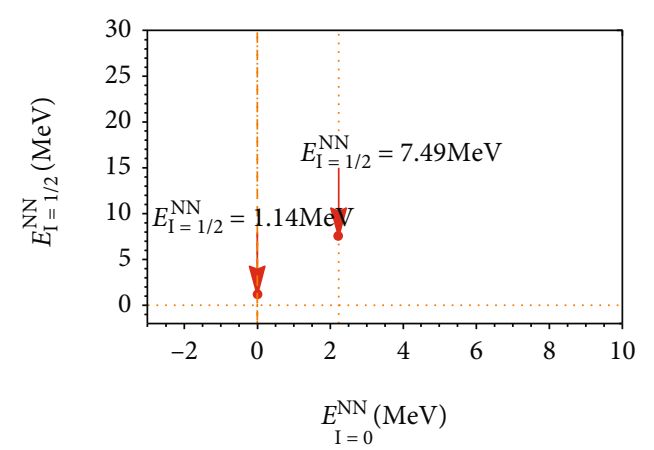

(b)

Figure 5: (a) Plot of wave functions for the $N N N$ system in the state $|0,1 / 2\rangle_{I} \otimes|1,1 / 2\rangle_{S}$. The blue lines denote the wave functions for any two nucleons in the NNN system. The red lines represent the wave functions for its subsystem deuteron. (b) Dependence of the total threebody binding energy on the two-body binding energy of its subsystem $N N$. The left red point indicates the Critical Borromean state of the system. The right one is our numerical result corresponding to the triton or helium-3 nucleus.

our calculations we use the BOP method to construct the interpolating wave functions for diagonalizing the Hamiltonian of the three-body system, it always gives an upper limit of the energy for a system.

3.3. Numerical Results for the $\Lambda \Lambda \Lambda$. There is only one possible state $|0,0\rangle_{I} \otimes|0,1 / 2\rangle_{S}$ for the system $\Lambda \Lambda \Lambda$, as $\Lambda$ is an isospin singlet. Now we only have a free parameter $\lambda$ which is undetermined in our calculations. In general, the parameter $\lambda$ is within the range $800-1500 \mathrm{MeV}$ when investigating the binding properties of deuteron. In our calculation for the $N N N$ system, it is chosen at $811.80 \mathrm{MeV}$ to reproduce the binding energy of deuteron within the OBE mechanism. One may expect that a heavier system has a smaller size, which leads to a larger parameter $\lambda$. Thus, we change the parameter $\lambda$ in the range of $800-2000 \mathrm{MeV}$ to search for the binding solutions of this system. It is crucial to investigate the two-body interaction before searching for the bound state of the system. The two-body force of the $\Lambda \Lambda \Lambda$ arises from the $\eta, \sigma, \omega$, and $\phi$ exchange. To highlight the contribution of each exchanged boson, we plot the total effective potential and the $\mathrm{S}$ wave effective potentials of each exchanged boson in Figure 6 when we fix the $\lambda$ at $811.80 \mathrm{MeV}$ for the state $|0,0\rangle_{I} \otimes|0,1 / 2\rangle_{S}$. Both the $\eta$ and $\omega$ exchanges provide repulsive force, while the $\sigma$ exchange is attractive. The $\phi$ exchange is attractive in the short range but repulsive in the medium range. The total effective potential is attractive.

Along with the procedure we used to discuss the threenucleon system, we first search the bound solutions for the subsystem $\Lambda \Lambda$. There is only one possible state $\left(I_{2}, S_{2}\right)=(0$ $, 0)$. However, we fail to find any bound solution for the two-body subsystem $\Lambda \Lambda$ within the range of $\lambda$. Since the lack of the tensor force, the $\Lambda \Lambda$ does not have deep enough attractions to form a bound state. The attraction between any two constituents is not strong enough to bind them. Thus, the three-body system $\Lambda \Lambda \Lambda$ is also unlikely to form a bound state.

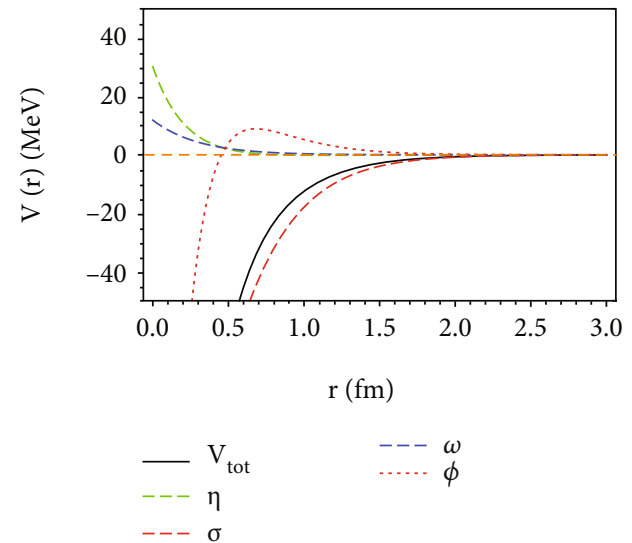

Figure 6: The $S$ wave effective potentials of the two-body force for the $\Lambda \Lambda \Lambda$ system in the state $|0,0\rangle_{\mathrm{I}} \otimes|0,1 / 2\rangle_{S}$ with the comparison of the effective potentials from each exchanged boson.

3.4. Numerical Results for the $\Xi \Xi \Xi$. There are four possible states for the $\Xi \Xi \Xi$ system, i.e., $|0,1 / 2\rangle_{I} \otimes|1,1 / 2\rangle_{S},|1,1 / 2\rangle_{I}$ $\otimes|0,1 / 2\rangle_{S},|0,1 / 2\rangle_{I} \otimes|1,3 / 2\rangle_{S}$, and $|1,3 / 2\rangle_{I} \otimes|0,1 / 2\rangle_{S}$. The general identity principle constricts the cases of the twobody force for the $\Xi \Xi \Xi$ system to be $(1,0)$ and $(0,1)$. The two-body force $(1,0)$ governs the states $|1,1 / 2\rangle_{I} \otimes|0,1 / 2\rangle_{S}$ and $|1,3 / 2\rangle_{I} \otimes|0,1 / 2\rangle_{S}$. The two-body force $(0,1)$ governs the states $|0,1 / 2\rangle_{I} \otimes|1,1 / 2\rangle_{S}$ and $|0,1 / 2\rangle_{I} \otimes|1,3 / 2\rangle_{S}$. Based on the OBE mechanism, the two-body force of the $\Xi \Xi \Xi$ is generated from the $\pi, \eta, \sigma, \rho, \omega$, and $\phi$ exchanges. We should search for the bound states of its two-body subsystem first, since one needs the two-body wave functions to construct the interpolating wave functions for the whole system within the BOP method. The Fourier transformation as Equation (31) yields the effective potentials of the two-body force in coordinate space, which is $\lambda$ dependent. With the variety of the parameter $\lambda$, we find binding solutions for both cases $(1,0)$ and $(0,1)$ of the subsystem $\Xi \Xi$ as shown in Tables 4 and 5. If the parameter $\lambda$ is chosen at $896.54 \mathrm{MeV}$, one finds a binding solution for the case $(0,1)$ with a binding energy of 
TABLE 4: Bound state solutions of the $\Xi \Xi \Xi$ system for the states $\left.\left|0,1 / 2_{I} \otimes\right| 1,1 / 2\right\rangle_{S}$ and $|0,1 / 2\rangle_{I} \otimes|1,3 / 2\rangle_{S}$. $E_{2}$ is the energy eigenvalue of its subsystem. $E_{3}$ is the reduced three-body energy eigenvalue relative to the breakup state of the $\Xi \Xi \Xi$ system. $E_{T}$ is the total three-body energy eigenvalue relative to the $\Xi \Xi \Xi$ threshold. $V_{\mathrm{BO}}(0)$ is the minimum of the BO potential. $r_{\mathrm{rms}}$ represents the root-mean-square radius of any two $\Xi$ in the $\Xi \Xi \Xi$ system. The $\mathrm{S}$ wave and $\mathrm{D}$ wave represent the probabilities for $\mathrm{S}$ wave and $\mathrm{D}$ wave components in any two $\Xi$ in the $\Xi \Xi \Xi$ system.

\begin{tabular}{lccccccc}
\hline$\Lambda(\mathrm{MeV})$ & $E_{2}(\mathrm{MeV})$ & $E_{3}(\mathrm{MeV})$ & $E_{T}(\mathrm{MeV})$ & $V_{\mathrm{BO}}(0)(\mathrm{MeV})$ & $\mathrm{S}$ wave $(\%)$ & $\mathrm{D}$ wave $(\%)$ & $r_{\text {rms }}(\mathrm{fm})$ \\
\hline .00 & -1.03 & -2.91 & -3.93 & -3.63 & 99.76 & 3.24 \\
.54 & -2.23 & -5.63 & -7.86 & -5.51 & 99.65 & 0.35 \\
.00 & -3.00 & -7.46 & -10.46 & -6.68 & 99.59 & 0.41 & 3.02 \\
.00 & -5.71 & -14.11 & -19.81 & -10.51 & 99.40 & 0.60 & 2.72 \\
.00 & -9.10 & -22.28 & -31.38 & -14.70 & 99.23 & 0.77 & 1.68 \\
.00 & -13.07 & -31.60 & -44.68 & -19.11 & 99.07 & 0.93 \\
.00 & -17.54 & -41.81 & -59.35 & -23.66 & 98.93 & 1.07 \\
.00 & -27.65 & -64.14 & -91.79 & -33.03 & 98.68 & 1.07 & 1.32 \\
\hline
\end{tabular}

TABLE 5: Bound state solutions of the $\Xi \Xi \Xi$ system for the states $|1,1 / 2\rangle_{I} \otimes|0,1 / 2\rangle_{S}$ and $|1,3 / 2\rangle_{I} \otimes|0,1 / 2\rangle_{S}$. $E_{2}$ is the energy eigenvalue of its subsystem. $E_{3}$ is the reduced three-body energy eigenvalue relative to the breakup state of the $\Xi \Xi \Xi$ system. $E_{T}$ is the total three-body energy eigenvalue relative to the $\Xi \Xi \Xi$ threshold. $V_{\mathrm{BO}}(0)$ is the minimum of the BO potential. $r_{\text {rms }}$ represents the root-mean-square radius of any two $\Xi$ in the $\Xi \Xi \Xi$ system. The $\mathrm{S}$ wave and $\mathrm{D}$ wave represent the probabilities for $\mathrm{S}$ wave and $\mathrm{D}$ wave components in any two $\Xi$ in the $\Xi \Xi \Xi$ system.

\begin{tabular}{lccccccc}
\hline$\Lambda(\mathrm{MeV})$ & $E_{2}(\mathrm{MeV})$ & $E_{3}(\mathrm{MeV})$ & $E_{T}(\mathrm{MeV})$ & $V_{\mathrm{BO}}(0)(\mathrm{MeV})$ & $\mathrm{S}$ wave $(\%)$ & $\mathrm{D}$ wave $(\%)$ & $r_{\text {rms }}(\mathrm{fm})$ \\
\hline .00 & -0.35 & -1.76 & -2.10 & -2.60 & 100.00 & 0 & 3.94 \\
.00 & -0.96 & -3.04 & -4.00 & -3.48 & 100.00 & 0 & 3.57 \\
.00 & -1.65 & -4.63 & -6.28 & -4.50 & 100.00 & 0 & 3.20 \\
.70 & -2.23 & -6.04 & -8.27 & -5.36 & 100.00 & 0 & 2.93 \\
.00 & -2.42 & -6.48 & -8.90 & -5.62 & 100.00 & 0 & 2.86 \\
.00 & -3.24 & -8.54 & -11.78 & -6.79 & 100.00 & 0 & 2.57 \\
.00 & -4.11 & -10.75 & -14.86 & -7.98 & 100.00 & 0 & 2.33 \\
.00 & -5.03 & -13.05 & -18.08 & -9.16 & 100.00 & 0 \\
\hline
\end{tabular}

2.23 MeV. The case $(1,0)$ also has a bound state with the same energy of $2.23 \mathrm{MeV}$, when the parameter $\lambda$ is fixed at 937.70 MeV.

In order to show the properties of the two-body interactions, we plot the $S$ wave effective potentials for both cases when their subsystem $\Xi \Xi$ has the same binding energy of $2.23 \mathrm{MeV}$ as shown in Figure 7. Figure 7(a) corresponds to the case $(0,1)$, where the $\pi, \omega$, and $\phi$ provide the repulsive force, while the $\eta, \rho$, and $\sigma$ give the attractive force. Since the repulsion of $\pi, \omega$, and $\phi$ exchanges almost cancel the attraction of $\eta$ and $\rho$, the total effective potential is dominated by the contribution of $\sigma$ exchange. We plot the effective potentials for the case $(1,0)$ in Figure $7(\mathrm{c})$, where the repulsion is mainly from $\pi, \eta, \rho$, and $\omega$ exchanges. The $\phi$ exchange provides a deep attraction in the short range but a slight repulsion in the medium range. The $\pi, \eta, \rho, \omega$, and $\phi$ almost cancel out and do not contribute the total effective potential. Thus, the $\sigma$ exchange mainly contributes the total effective potential. Based on the formalism discussed in Section 2, we get the BO potentials for the $\Xi \Xi \Xi$ system as shown in Figures $7(\mathrm{~b})$ and $7(\mathrm{~d})$, where (b) and (d) correspond to the cases of $(0,1)$ and $(1,0)$, respectively. Similar with the discussions on the three-nucleon system, the cor- rections on the interpolating wave functions weaken the BO potentials to some extent.

When the parameter $\lambda$ lies between $890.00 \mathrm{MeV}$ and $960.00 \mathrm{MeV}$, there is bound solution for the state $|0,1 / 2\rangle_{I}$ $\otimes|1,1 / 2\rangle_{S}\left(|0,1 / 2\rangle_{I} \otimes|1,3 / 2\rangle_{S}\right)$ with the total binding energies between $3.93 \mathrm{MeV}$ and $91.79 \mathrm{MeV}$ as shown in Table 4. The corresponding two-body binding energy is about $1.03-27.65 \mathrm{MeV}$. The reduced three-body binding energy increases from $2.91 \mathrm{MeV}$ to $64.14 \mathrm{MeV}$ as the parameter $\lambda$ grows, while the root-mean-square radius of the system decreases from $3.62 \mathrm{fm}$ to $1.09 \mathrm{fm}$. The three-body bound state of the state $|0,1 / 2\rangle_{I} \otimes|1,1 / 2\rangle_{S}$ $\left(|0,1 / 2\rangle_{I} \otimes|1,3 / 2\rangle_{S}\right)$ is a mixture of the $\mathrm{S}$ and $\mathrm{D}$ wave due to the tensor force in the effective potentials. The proportion of the $S$ wave state is more than $98 \%$. For a better comparison with the numerical results of the NNN system, we choose the parameter $\lambda$ at $896.54 \mathrm{MeV}$ which yields a twobody binding energy of $2.23 \mathrm{MeV}$ for the subsystem $\Xi \Xi$. Then, the total three-body binding energy and the reduced three-body binding energy are 7.86 and $5.63 \mathrm{MeV}$, respectively. Since we do not consider the three-body force in our calculation, the states $|0,1 / 2\rangle_{I} \otimes|1,1 / 2\rangle_{S}$ and $|0,1 / 2\rangle_{I}$ $\otimes|1,3 / 2\rangle_{S}$ are degenerate. 


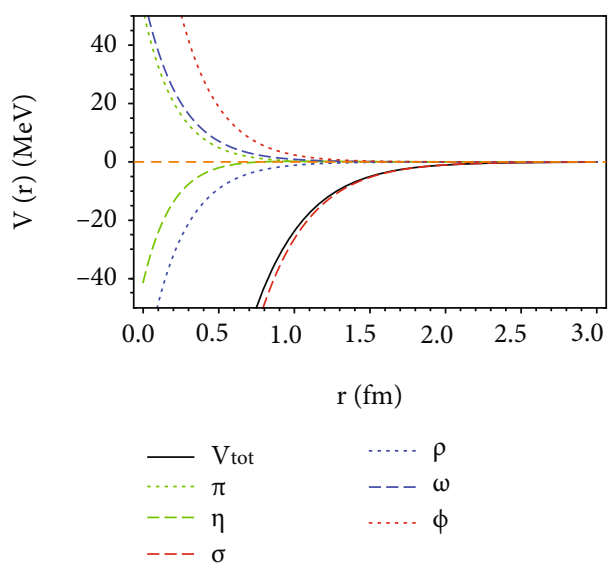

(a)

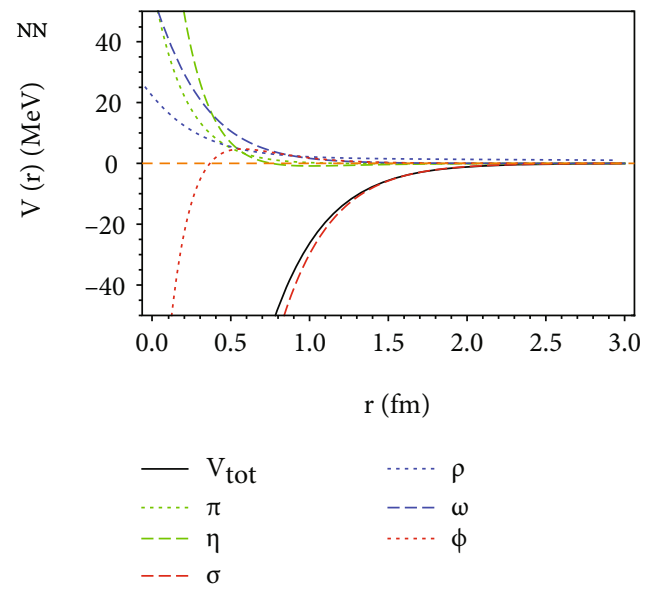

(c)
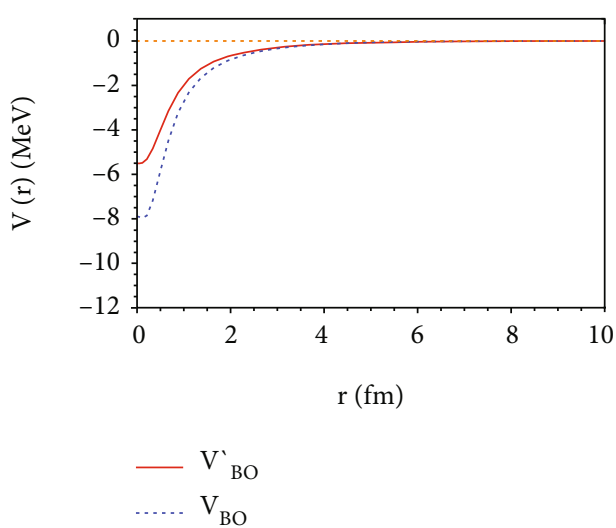

(b)

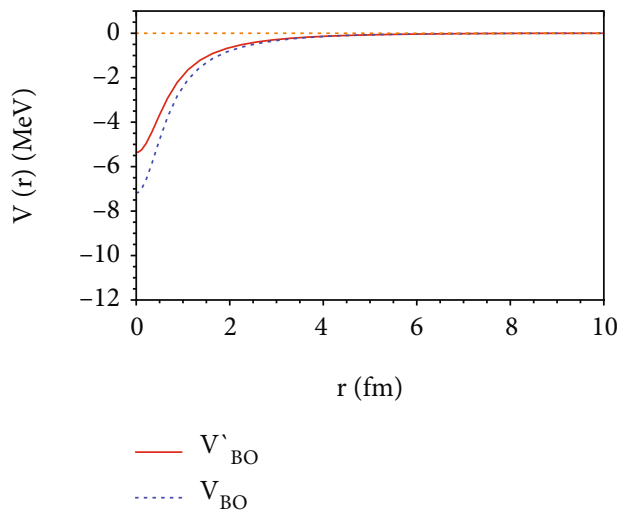

(d)

Figure 7: (a) The S wave effective potentials of the two-body force $(0,1)$ for the system $\Xi \Xi \Xi$ with the comparison of the contributions of each exchanged boson. (b) The BO potentials for the system $\Xi \Xi \Xi$ provided by the two-body force $(0,1)$. (c) The $S$ wave effective potentials of the two-body force $(1,0)$ for the system $\Xi \Xi \Xi$ with the comparison of the contributions of each exchanged boson. (d) The BO potentials for the system $\Xi \Xi \Xi$ provided by the two-body force $(1,0)$. The dotted and solid lines in (b) and (d) are the BO potentials before and after distortion correction, respectively.

When the parameter $\lambda$ increases between $910.00 \mathrm{MeV}$ and $970.00 \mathrm{MeV}$, we find a degenerate bound solution for the states $|1,1 / 2\rangle_{I} \otimes|0,1 / 2\rangle_{S}$ and $|1,3 / 2\rangle_{I} \otimes|0,1 / 2\rangle_{S}$. The corresponding two-body binding energy is about 1.11 6.19 MeV, and their three-body binding energies vary from $2.10 \mathrm{MeV}$ to $18.08 \mathrm{MeV}$. The reduced three-body binding energy grows from $1.76 \mathrm{MeV}$ to $13.05 \mathrm{MeV}$ with the parameter $\lambda$ in the range of $910.00-970.00 \mathrm{MeV}$. The root-meansquare radius of the system decreases from $3.94 \mathrm{fm}$ to $2.13 \mathrm{fm}$. The three-body bound states of the states $|1,1 / 2\rangle_{I}$ $\otimes|0,1 / 2\rangle_{S}$ and $|1,3 / 2\rangle_{I} \otimes|0,1 / 2\rangle_{S}$ only have $S$ wave state due to their effective potentials have no tensor force, which are different from the cases $|0,1 / 2\rangle_{I} \otimes|1,1 / 2\rangle_{S}$ and $|0,1 / 2\rangle_{I}$ $\otimes|1,3 / 2\rangle_{S}$. From Table 2, we can see that the total threebody binding energy of the states $|1,1 / 2\rangle_{I} \otimes|0,1 / 2\rangle_{S}$ and $|1,3 / 2\rangle_{I} \otimes|0,1 / 2\rangle_{S}$ is about $8.27 \mathrm{MeV}$ when the two-body binding energy is $2.23 \mathrm{MeV}$ with the corresponding parameter $\lambda$ is fixed at $937.70 \mathrm{MeV}$.
We plot the wave functions for the $\Xi \Xi \Xi$ in Figures 8(a) and 8 (c) to verify the solutions we get are bound states. The wave functions in Figure $8(\mathrm{a})$ correspond to the state $|0,1 / 2\rangle_{I} \otimes|1,1 / 2\rangle_{S}\left(|0,1 / 2\rangle_{I} \otimes|1,3 / 2\rangle_{S}\right)$, while the wave functions in Figure $8(\mathrm{c})$ correspond to the state $|1,1 / 2\rangle_{I} \otimes$ $|0,1 / 2\rangle_{S}\left(|1,3 / 2\rangle_{I} \otimes|0,1 / 2\rangle_{S}\right)$. We plot the dependence of the three-body binding energy of the $\Xi \Xi \Xi$ system on its corresponding two-body binding energy in Figures 8 (b) and 8 (d), where Figures $8(\mathrm{~b})$ and $8(\mathrm{~d})$ correspond to state $|0,1 / 2\rangle_{I} \otimes|1,1 / 2\rangle_{S}\left(|0,1 / 2\rangle_{I} \otimes|1,3 / 2\rangle_{S}\right)$ and $|1,1 / 2\rangle_{I} \otimes|0,1 / 2\rangle_{S}\left(|1,3 / 2\rangle_{I} \otimes|0,1 / 2\rangle_{S}\right), \quad$ respectively. Similar with the three-nucleon system, the $\Xi \Xi \Xi$ system for both cases also has a Critical Borromean state. There are two red points in both figures, where the left one indicates the Critical Borromean state of the system. For the state $|0,1 / 2\rangle_{I} \otimes|1,1 / 2\rangle_{S}\left(|0,1 / 2\rangle_{I} \otimes|1,3 / 2\rangle_{S}\right)$ as shown in Figure $8(\mathrm{~b})$, there is still a shallow three-body bound state with a tiny binding energy of $1.06 \mathrm{MeV}$ when the two-body 


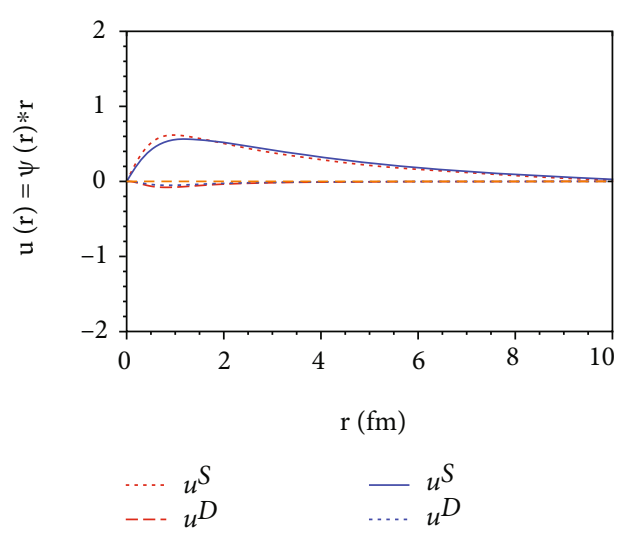

(a)

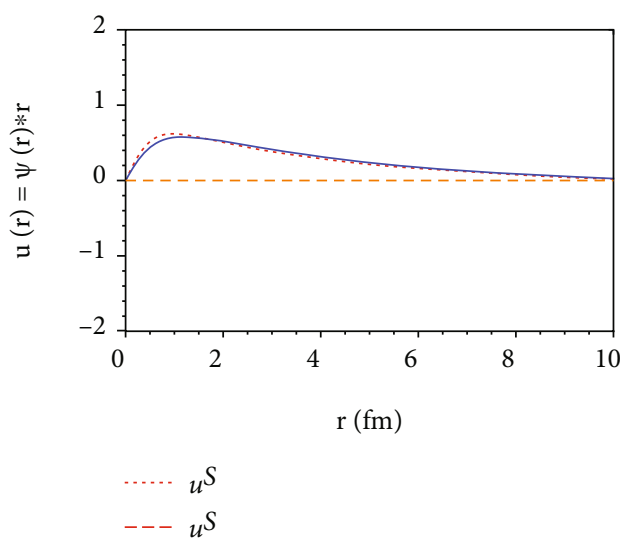

(c)

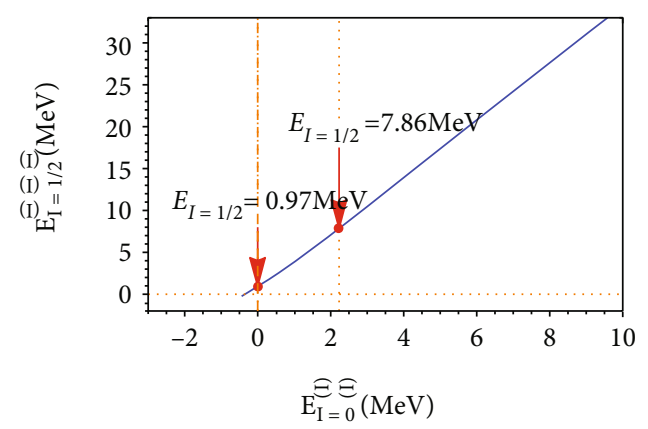

(b)

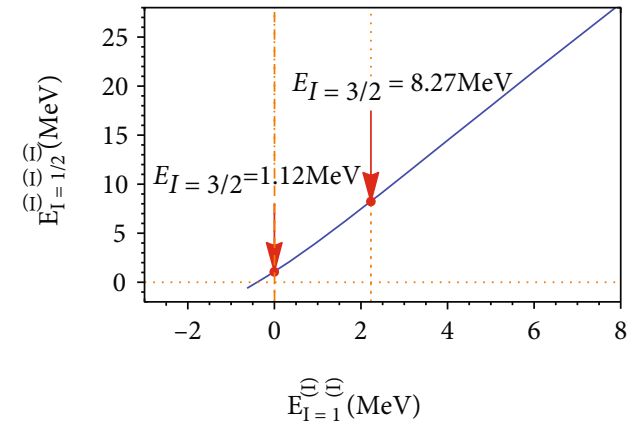

(d)

Figure 8: (a) Plot of wave functions for the $\Xi \Xi \Xi$ system in the state $|0,1 / 2\rangle_{I} \otimes|1,1 / 2\rangle_{S}\left(|0,1 / 2\rangle_{I} \otimes|1,3 / 2\rangle_{S}\right)$. The blue lines denote the wave functions for any two $\Xi$ in the $\Xi \Xi \Xi$ system. The red lines represent the wave functions for its subsystem $\Xi \Xi$. (b) Dependence of the total three-body binding energy on the two-body binding energy of its subsystem $\Xi \Xi$ for the state $|0,1 / 2\rangle_{I} \otimes|1,1 / 2\rangle_{S}\left(|0,1 / 2\rangle_{I} \otimes|1,3 / 2\rangle_{S}\right)$. The left red point indicates the Critical Borromean state of the system. The right one is our numerical result when the two-body binding energy is chosen at $2.23 \mathrm{MeV}$. (c) Plot of wave functions for the $\Xi \Xi \Xi$ system in the state $|1,1 / 2\rangle_{I} \otimes|0,1 / 2\rangle_{S}\left(|1,3 / 2\rangle_{I} \otimes|0,1 / 2\rangle_{S}\right)$. (d) Dependence of the total three-body binding energy on the two-body binding energy of its subsystem $\Xi \Xi$ for the state $|1, / 2\rangle_{I} \otimes$ $|0, / 2\rangle_{S}\left(|1,3 / 2\rangle_{I} \otimes|0, / 2\rangle_{S}\right)$.

binding energy approaches to $0 \mathrm{MeV}$. Similarly, the isospin state $|1,1 / 2\rangle_{I} \otimes|0,1 / 2\rangle_{S}\left(|1,3 / 2\rangle_{I} \otimes|0,1 / 2\rangle_{S}\right)$ has a threebody bound state with a tiny binding energy of $1.12 \mathrm{MeV}$ when the two-body binding energy approaches to $0 \mathrm{MeV}$. The right red points in both figures represent our numerical results when the two-body binding energy is chosen at $2.23 \mathrm{MeV}$. Since $\Xi$ is a little heavier than nucleon, the three-body bound state for the $\Xi \Xi \Xi$ system is more likely to have a deeper bound state.

3.5. Numerical Results for the $\Sigma \Sigma \Sigma$. The possible states of the $\Sigma \Sigma \Sigma$ system could be $|0,1\rangle_{I} \otimes|0,1 / 2\rangle_{S}, \quad|1,(0,1,2)\rangle_{I} \otimes$ $|1,1 / 2(3 / 2)\rangle_{S}$, and $|2,(1,2,3)\rangle_{I} \otimes|0,1 / 2\rangle_{S}$. Since the constraint from the general identity principle, there are three possible two-body force for the $\Sigma \Sigma \Sigma$ system, i.e., $(0,0)$, $(1,1)$, and $(2,0)$. The state $|0,1\rangle_{I} \otimes|0,1 / 2\rangle_{S}$ is governed by the two-body force $(0,0)$. The state $|1,(0,1,2)\rangle_{I} \otimes$ $|1,1 / 2(3 / 2)\rangle_{S}$ is governed by the two-body force $(1,1)$. The state $|2,(1,2,3)\rangle_{I} \otimes|0,1 / 2\rangle_{S}$ is dominated by the two-body force $(2,0)$. The $\pi, \eta, \sigma, \rho, \omega$, and $\phi$ exchanges all contribute to the total effective potentials. Based on the formalism we constructed in Section 2, we need to search for the twobody bound states of its subsystem first. The effective potentials for the subsystem $\Sigma \Sigma$ can be derived from the Lagrangians in Equation (29). After the Fourier transformation in Equation (31), we get the effective potentials of the twobody force in coordinate space, which rely on the parameter $\lambda$. When the parameter $\lambda$ is more than certain value, we find bound solutions for all of three cases as shown in Tables 6-8. For the case $(0,0)$, we find a bound state with a binding energy of $2.23 \mathrm{MeV}$ when the parameter $\lambda$ is fixed at $741.66 \mathrm{MeV}$. For the case $(1,1)$, there is a bound state with a binding energy of $2.23 \mathrm{MeV}$ when the parameter $\lambda$ is chosen at $915.71 \mathrm{MeV}$. It turns out a bound state with the same binding energy for the case $(2,0)$ when we fix the parameter $\lambda$ at $1104.42 \mathrm{MeV}$.

To make the individual role of the exchanged bosons clear, we plot the $S$ wave effective potentials for all of the three cases when their subsystem $\Sigma \Sigma$ has the same binding energy of $2.23 \mathrm{MeV}$. We show them in Figure 9, where (a), (b), and (c) correspond to the cases $(0,0),(1,1)$, and $(2,0)$, respectively. In Figure $9(\mathrm{a})$, the $\eta, \rho$, and $\phi$ exchanges are 
TABLE 6: Bound state solutions of the $\Sigma \Sigma \Sigma$ system in the state $|0,1\rangle_{I} \otimes|0,1 / 2\rangle_{S} . E_{2}$ is the energy eigenvalue of its subsystem. $E_{3}$ is the reduced three-body energy eigenvalue relative to the breakup state of the $\Sigma \Sigma \Sigma$ system. $E_{T}$ is the total three-body energy eigenvalue relative to the $\Sigma \Sigma \Sigma$ threshold. $V_{\mathrm{BO}}(0)$ is the minimum of the BO potential. $r_{\text {rms }}$ represents the root-mean-square radius of any two $\Sigma$ in the $\Sigma \Sigma \Sigma$ system. The $\mathrm{S}$ wave and $\mathrm{D}$ wave represent the probabilities for $\mathrm{S}$ wave and $\mathrm{D}$ wave components in any two $\Sigma$ in the $\Sigma \Sigma \Sigma$ system.

\begin{tabular}{|c|c|c|c|c|c|c|c|}
\hline$\Lambda(\mathrm{MeV})$ & $E_{2}(\mathrm{MeV})$ & $E_{3}(\mathrm{MeV})$ & $E_{T}(\mathrm{MeV})$ & $V_{\mathrm{BO}}(0)(\mathrm{MeV})$ & $\mathrm{S}$ wave $(\%)$ & D wave (\%) & $r_{\mathrm{rms}}(\mathrm{fm})$ \\
\hline .00 & -0.49 & -1.89 & -2.38 & -4.02 & 100.00 & 0 & 3.91 \\
\hline .00 & -1.75 & -4.83 & -6.57 & -6.40 & 100.00 & 0 & 3.21 \\
\hline .66 & -2.23 & -6.08 & -8.31 & -7.33 & 100.00 & 0 & 2.98 \\
\hline .00 & -3.32 & -8.97 & -12.29 & -9.30 & 100.00 & 0 & 2.57 \\
\hline .00 & -5.21 & -14.07 & -19.28 & -12.42 & 100.00 & 0 & 2.09 \\
\hline .00 & -7.37 & -19.86 & -27.24 & -15.62 & 100.00 & 0 & 1.78 \\
\hline .00 & -9.78 & -26.18 & -35.96 & -18.85 & 100.00 & 0 & 1.56 \\
\hline .00 & -12.42 & -32.91 & -45.33 & -22.11 & 100.00 & 0 & 1.41 \\
\hline
\end{tabular}

TABLE 7: Bound state solutions of the $\Sigma \Sigma \Sigma$ system in the state $|1,(0,1,2)\rangle_{I} \otimes|1,1 / 2(3 / 2)\rangle_{S}$. $E_{2}$ is the energy eigenvalue of its subsystem. $E_{3}$ is the reduced three-body energy eigenvalue relative to the breakup state of the $\Sigma \Sigma \Sigma$ system. $E_{T}$ is the total three-body energy eigenvalue relative to the $\Sigma \Sigma \Sigma$ threshold. $V_{\mathrm{BO}}(0)$ is the minimum of the BO potential. $r_{\text {rms }}$ represents the root-mean-square radius of any two $\Sigma$ in the $\Sigma \Sigma \Sigma$ system. The $\mathrm{S}$ wave and $\mathrm{D}$ wave represent the probabilities for $\mathrm{S}$ wave and $\mathrm{D}$ wave components in any two $\Sigma$ in the $\Sigma \Sigma \Sigma$ system.

\begin{tabular}{lccccccc}
\hline$\Lambda(\mathrm{MeV})$ & $E_{2}(\mathrm{MeV})$ & $E_{3}(\mathrm{MeV})$ & $E_{T}(\mathrm{MeV})$ & $V_{\mathrm{BO}}(0)(\mathrm{MeV})$ & S wave $(\%)$ & $\mathrm{D}$ wave $(\%)$ & $r_{\text {rms }}(\mathrm{fm})$ \\
\hline .00 & -1.11 & -2.87 & -3.98 & -3.99 & 99.11 & 0.89 & 3.78 \\
.00 & -1.80 & -4.35 & -6.15 & -5.08 & 98.94 & 1.06 \\
.71 & -2.24 & -5.31 & -7.55 & -5.76 & 98.84 & 1.16 \\
.00 & -2.58 & -6.08 & -8.66 & -6.29 & 98.77 & 1.23 \\
.00 & -3.42 & -8.02 & -11.43 & -7.57 & 98.61 & 3.25 \\
.00 & -4.31 & -10.10 & -14.41 & -8.87 & 98.45 & 1.39 \\
.00 & -5.24 & -12.29 & -17.53 & -10.18 & 98.31 & 1.55 \\
.00 & -6.19 & -14.53 & -20.72 & -11.47 & 98.18 & 1.69 & 2.81 \\
\hline
\end{tabular}

TABLE 8: Bound state solutions of the $\Sigma \Sigma \Sigma$ system in the state $|2,(1,2,3)\rangle_{I} \otimes|0,1 / 2\rangle_{S} . E_{2}$ is the energy eigenvalue of its subsystem. $E_{3}$ is the reduced three-body energy eigenvalue relative to the breakup state of the $\Sigma \Sigma \Sigma$ system. $E_{T}$ is the total three-body energy eigenvalue relative to the $\Sigma \Sigma \Sigma$ threshold. $V_{\mathrm{BO}}(0)$ is the minimum of the $\mathrm{BO}$ potential. $r_{\text {rms }}$ represents the root-mean-square radius of any two $\Sigma$ in the $\Sigma \Sigma \Sigma$ system. The $\mathrm{S}$ wave and $\mathrm{D}$ wave represent the probabilities for $\mathrm{S}$ wave and $\mathrm{D}$ wave components in any two $\Sigma$ in the $\Sigma \Sigma \Sigma$ system.

\begin{tabular}{lccccccc}
\hline$\Lambda(\mathrm{MeV})$ & $E_{2}(\mathrm{MeV})$ & $E_{3}(\mathrm{MeV})$ & $E_{T}(\mathrm{MeV})$ & $V_{\mathrm{BO}}(0)(\mathrm{MeV})$ & $\mathrm{S}$ wave $(\%)$ & $\mathrm{D}$ wave $(\%)$ & $r_{\text {rms }}(\mathrm{fm})$ \\
\hline .00 & -0.85 & -1.23 & -2.09 & -5.30 & 100.00 & 0 & 4.43 \\
.42 & -2.23 & -3.63 & -5.86 & -9.27 & 100.00 & 0 & 3.84 \\
.00 & -2.45 & -4.07 & -6.52 & -9.96 & 100.00 & 0 & 3.74 \\
.00 & -4.86 & -9.60 & -14.46 & -17.51 & 100.00 & 0 & 0 \\
.00 & -8.20 & -18.91 & -27.11 & -27.42 & 100.00 & 1.98 \\
.00 & -12.53 & -32.12 & -44.65 & -38.78 & 100.00 & 0 \\
\hline
\end{tabular}

repulsive, while the $\pi$ and $\sigma$ provide attractions. The $\omega$ exchange gives a shallow repulsion in the medium range but attraction in the short range. In Figure 9(b), the $\pi, \omega$ and $\phi$ exchanges provide repulsions, while the $\eta, \rho$ and $\sigma$ exchanges are attractive. In Figure 9(c), the $\eta$ and $\phi$ exchanges are repulsive, while the $\sigma$ exchange is attractive. The $\rho$ and $\omega$ provide deep attractions in the short range but strong repulsion in the medium range. The $\pi$ exchange is repulsive in the short range but slightly attractive in the medium range. We also plot the corresponding BO potentials for the three cases in Figures 9(d)-9(f). Similarly, the $\mathrm{BO}$ potentials become shallow after distortion corrections.
A three-body bound state appears for the state $|0,1\rangle_{I} \otimes$ $|0,1 / 2\rangle_{S}$ with the total binding energy about 2.38$45.33 \mathrm{MeV}$, when the parameter is around 735.00$765.00 \mathrm{MeV}$ as shown in Table 6. The corresponding twobody binding energy of its two-body subsystem is around 0.49-12.42 MeV. The root-mean-square radius of the system decreases from $3.91 \mathrm{fm}$ to $1.41 \mathrm{fm}$. Since the potentials in this case do not contain tensor force, the three-body binding solution is dominated by the $S$ wave state. For comparison with the numerical results of the $N N N$ and $\Xi \Xi \Xi$ systems, we fix the two-body binding energy at $2.23 \mathrm{MeV}$ which yields a total three-body binding energy of $8.31 \mathrm{MeV}$ with the parameter $\lambda=741.66 \mathrm{MeV}$. 


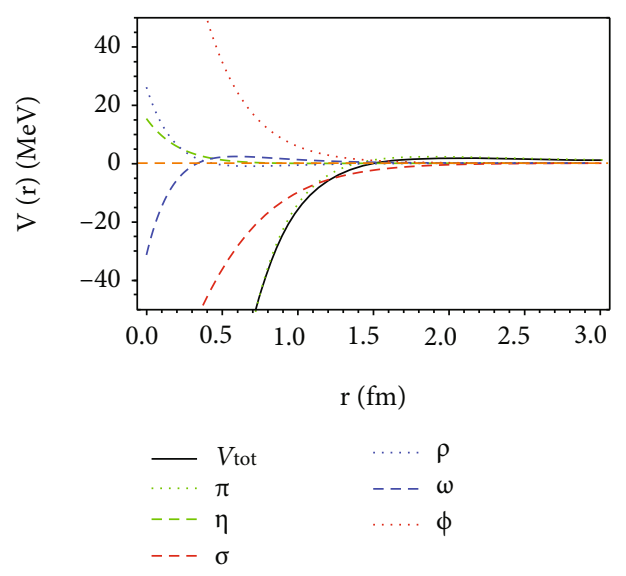

(a)

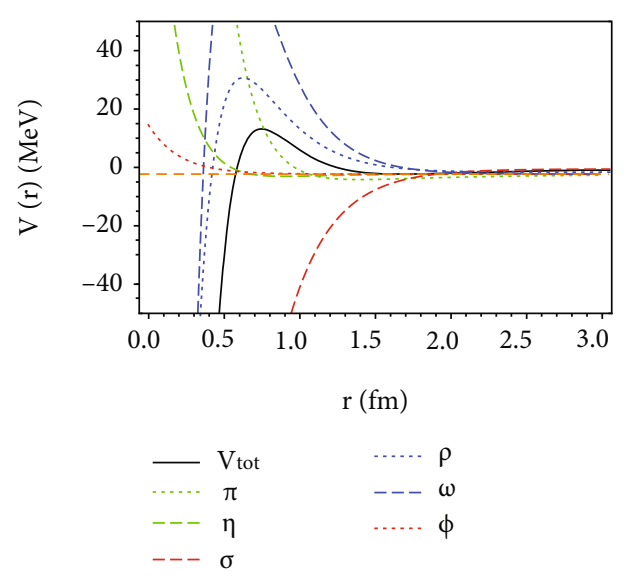

(c)

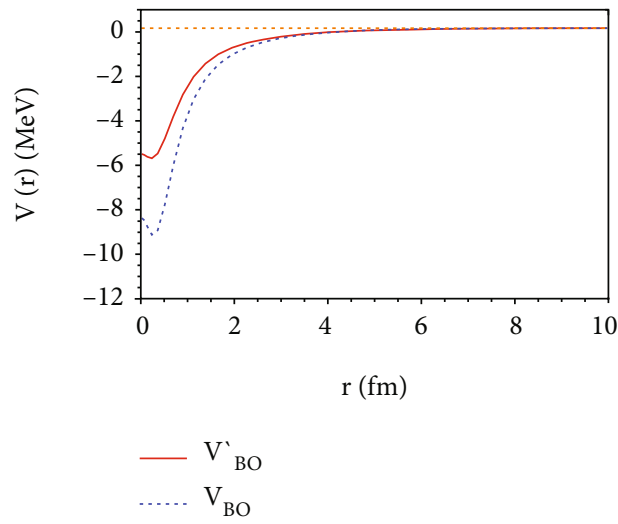

(e)

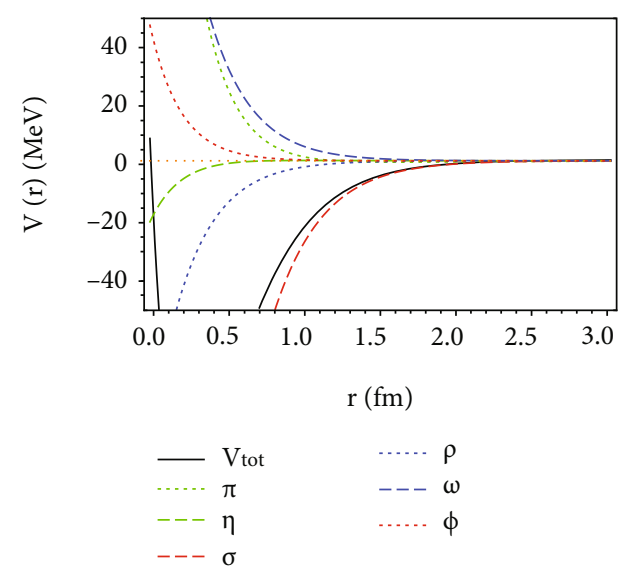

(b)
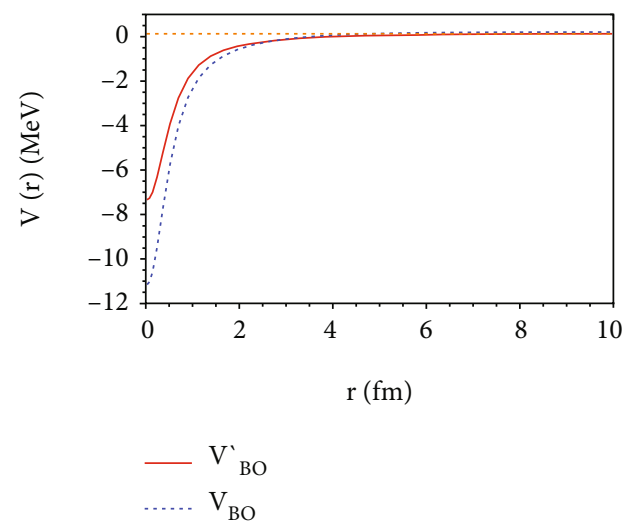

(d)

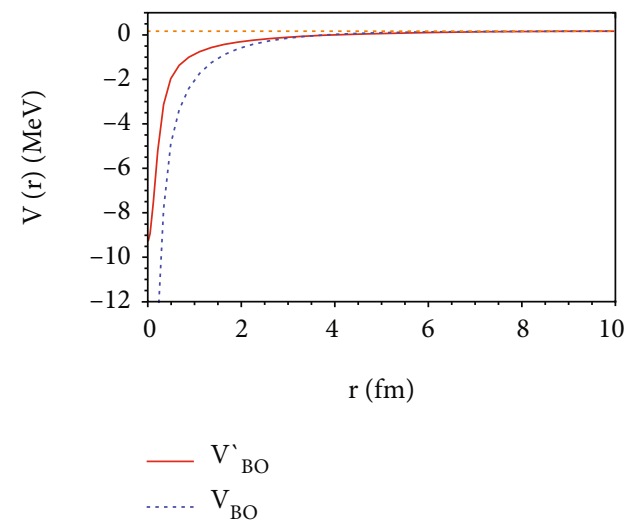

(f)

Figure 9: $(\mathrm{a}-\mathrm{c})$ The plots of the $\mathrm{S}$ wave effective potentials of the two-body force $(0,0),(1,1)$, and $(2,0)$ for the system $\Sigma \Sigma \Sigma$, respectively. (df) Plots of the BO potentials for the system $\Sigma \Sigma \Sigma$ provided by the two-body force $(0,0),(1,1)$, and $(2,0)$, respectively. The dotted and solid lines in $(\mathrm{d}-\mathrm{f})$ are the BO potentials before and after distortion corrections, respectively.

After solving the three-body equation in Equation (21), we find that the state $|1,(0,1,2)\rangle_{I} \otimes|1,1 / 2(3 / 2)\rangle_{S}$ has a three-body bound state as shown in Table 7. It is a degenerate state due to the negligence of three-body force in our formalism. The total binding energy of the $\Sigma \Sigma \Sigma$ corresponding to the $|1,(0,1,2)\rangle_{I} \otimes|1,1 / 2(3 / 2)\rangle_{S}$ state is about $3.98-$ $20.72 \mathrm{MeV}$ when the parameter $\lambda$ is around 900.00-
$960.00 \mathrm{MeV}$. The corresponding two-body binding energy of its two-body subsystem is around $1.11-6.19 \mathrm{MeV}$. The root-mean-square radius of the system in this isospin state decreases from $3.78 \mathrm{fm}$ to $2.19 \mathrm{fm}$. Since the existence of the tensor force in the effective potentials, the $\mathrm{S}$-D wave mixing should be taken into account. Within the variety of the parameter $\lambda$, one can see that the bound state is dominated 
by the $S$ wave state, which has a proportion more than $98 \%$. If we choose the parameter $\lambda$ at $915.71 \mathrm{MeV}$, then the total three-body binding energy is fixed at $\mathrm{MeV}$ with the corresponding two-body binding energy of $2.23 \mathrm{MeV}$.

We also find that all of the states $|2,1\rangle_{I} \otimes|0,1 / 2\rangle_{S}$, $|2,2\rangle_{I} \otimes|0,1 / 2\rangle_{S}$ and $|2,3\rangle_{I} \otimes|0,1 / 2\rangle_{S}$ have a degenerate three-body bound state as shown in Table 8 . Similarly, we write the states $|2,1\rangle_{I} \otimes|0,1 / 2\rangle_{S}, \quad|2,2\rangle_{I} \otimes|0,1 / 2\rangle_{S}$ and $|2,3\rangle_{I} \otimes|0,1 / 2\rangle_{S}$ together as $|2,(1,2,3)\rangle_{I} \otimes|0,1 / 2\rangle_{S}$ for simplicity. When the parameter $\lambda$ is about $1100.00-$ $1120.00 \mathrm{MeV}$, the three-body binding energy is around 2.09-44.65 MeV. The corresponding two-body binding energy of the two-body subsystem is around 0.85$12.53 \mathrm{MeV}$. The root-mean-square radius of the system decreases from $4.43 \mathrm{fm}$ to $1.47 \mathrm{fm}$. Similar with the case of the $|0,1\rangle$ state, the binding solution only has the $S$ wave state. The total three-body binding energy is fixed at $5.86 \mathrm{MeV}$ with the same two-body binding energy of $2.23 \mathrm{MeV}$, when the parameter $\lambda$ is $1104.42 \mathrm{MeV}$.

The wave functions for the $\Sigma \Sigma \Sigma$ system are shown in Figures $10(\mathrm{a})-10(\mathrm{c})$, where (a), (b), and (c) are corresponding to the states $|0,1\rangle_{I} \otimes|0,1 / 2\rangle_{S}, \quad|1,(0,1,2)\rangle_{I} \otimes$ $|1,1 / 2(3 / 2)\rangle_{S}$ and $|2,(1,2,3)\rangle_{I} \otimes|0,1 / 2\rangle_{S}$, respectively. The numerical results show that the three-body binding energy increases as the two-body binding energy increases. One may wonder whether there is a Critical Borromean state for the $\Sigma \Sigma \Sigma$ system. After lots of calculations, it turns out that the Critical Borromean states for all of the isospin states are existent. We label two red points in all of the figures, where the left one indicates the Critical Borromean state of the system. In Figure 10(d), we can see that the Critical Borromean state of the $|0,1\rangle_{I} \otimes|0,1 / 2\rangle_{S}$ state has a small threebody binding energy of $1.07 \mathrm{MeV}$ when the two-body binding energy approaches to $0 \mathrm{MeV}$. The isospin state $|1,(0,1,2)\rangle_{I} \otimes|1,1 / 2(3 / 2)\rangle_{S}$ also has a small three-body binding energy of $1.06 \mathrm{MeV}$ when we decrease the twobody binding energy to $0 \mathrm{MeV}$. Similarly, it appears a Critical Borromean state for the $|2,(1,2,3)\rangle_{I} \otimes|0,1 / 2\rangle_{S}$ state when the two-body binding energy approaches to $0 \mathrm{MeV}$. The right red points in the three figures represent our numerical results when the two-body binding energy is chosen at $2.23 \mathrm{MeV}$. Since our formalism is a version of variational principle, the numerical results of the three-body binding energies should be lower than the strict values.

\section{Summary}

The contradiction between the reported "XYZ" states and the traditional quark model arouses interest in searching for the possible deuteronlike molecules. Among all of the theoretical interpretations, the molecule scenario becomes more and more popular and has been identified to be a good candidate to interpret some of the "XYZ" states. The molecular structures of triton and helium-3 nucleus inspire us to study the tritonlike systems. First, we have constructed the formalism of the BOP method for a general system consists of the three identical fermions with half spin. Then, based on the BOP method as well as the OBE model, we have per- formed an extensive investigation on the tritonlike systems composed of three identical hadrons of baryon octet, i.e., the $\Lambda \Lambda \Lambda, \Xi \Xi \Xi$, and $\Sigma \Sigma \Sigma$. In our formalism, there is only one free parameter $\lambda$ introduced in the monopole form factor to suppress the contribution from UV energies. The parameter $\lambda$ is hard to be pinned down from fundamental theories. Thus, we choose the parameter $\lambda$ in a reasonable range to show various binding solutions of the tritonlike systems. Before calculations on the trihyperon systems, we apply our formalism on the NNN system to verify the feasibility of the BOP method. We find a binding solution in the state $|0,1 / 2\rangle_{I} \otimes|1,1 / 2\rangle_{S}$ with a total binding energy of $7.49 \mathrm{MeV}$, which is comparable with empirical binding energies of the triton and helium-3 nucleus. In our calculations, there is no numerical difference between the binding energies of the triton and helium-3 nucleus, as we do not consider the isospin breaking effect.

Then, we return to the calculations on the trihyperon system. For the $\Lambda \Lambda \Lambda$ system, we fail to find any bound solution with the variety of the parameter $\lambda$. After the treatments of the S-D wave mixing within the OBE mechanism, we find that all of the isospin eigenstates have bound state solutions for the $\Xi \Xi \Xi$ system. For better comparisons with the threenucleon system, we fix the two-body binding energy of its subsystem at $2.23 \mathrm{MeV}$ which is the same with deuteron. Then, the total binding energy of the $\Xi \Xi \Xi$ system in the state $|0,1 / 2\rangle_{I} \otimes|1,1 / 2\rangle_{S}\left(|0,1 / 2\rangle_{I} \otimes|1,3 / 2\rangle_{S}\right)$ is $7.86 \mathrm{MeV}$, while it is $8.27 \mathrm{MeV}$ for the state $|1,1 / 2\rangle_{I} \otimes|0,1 / 2\rangle_{S}$ $\left(|1,3 / 2\rangle_{I} \otimes|0,1 / 2\rangle_{S}\right)$. A three-body bound state appears for the $\Sigma \Sigma \Sigma$ system in all of the isospin eigenstates as the parameter $\lambda$ grows. When the two-body binding energy is chosen at $2.23 \mathrm{MeV}$, the total binding energy of the $\Sigma \Sigma \Sigma$ system in the state $|0,1\rangle_{I} \otimes|0,1 / 2\rangle_{S}$ is $8.31 \mathrm{MeV}$. For the isospin states $|1,(0,1,2)\rangle_{I} \otimes|1,1 / 2(3 / 2)\rangle_{S}$ and $|2,(1,2,3)\rangle_{I} \otimes$ $|0,1 / 2\rangle_{S}$, the total binding energies are $7.55 \mathrm{MeV}$ and $5.86 \mathrm{MeV}$, respectively. Since the total binding energy of the trihyperon system depends on the two-body binding energy of their corresponding subsystems, we plot the dependence lines for each systems. All of the $N N N, \Xi \Xi \Xi$, and $\Sigma \Sigma \Sigma$ have a Critical Borromean state when the twobody binding energies of their corresponding two-body subsystems approach to $0 \mathrm{MeV}$. It means that no matter how small their two-body binding energies are, as long as their corresponding two-body subsystems can form bound states, the three-body bound states are most likely to exist.

In our formalism, we use the $\mathrm{BO}$ potential to describe the contribution of one of the particles on the dynamics of the other two. Through the BOP method, one can divide a three-body system into three two-body systems with the BO potentials created by the omitted particles. The three different simplification schemes lead to the three different configurations. After simplest combinations of the three configurations, we construct the interpolating wave functions for diagonalizing the Hamiltonian of the three-body system. In fact, the strict solutions should be the a more complicated combinations of all possible configurations, which require further investigations. One should notice that the BOP method we have used is a version of the variational principle. It always gives an upper limit of the energy for a system. Therefore, 


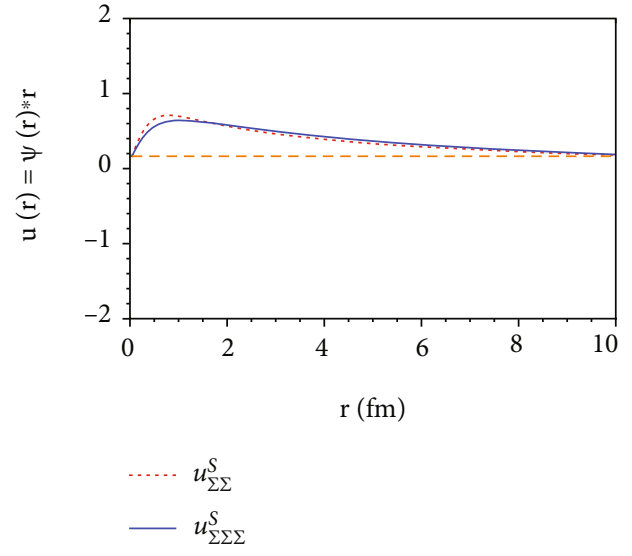

(a)

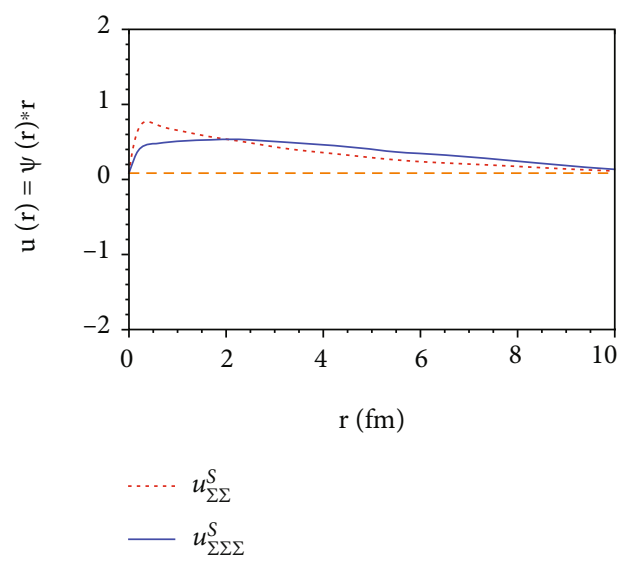

(c)

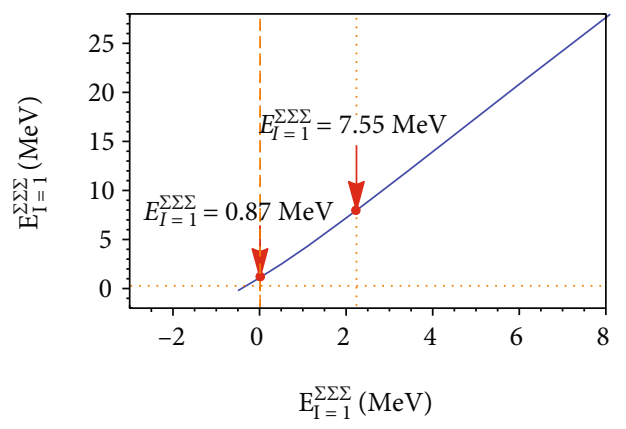

(e)

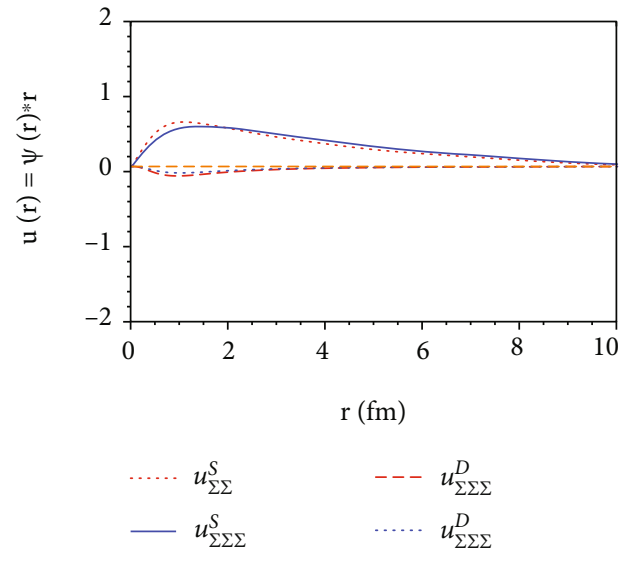

(b)

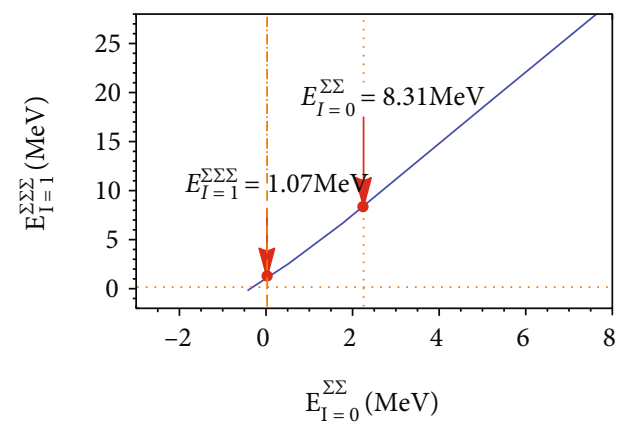

(d)

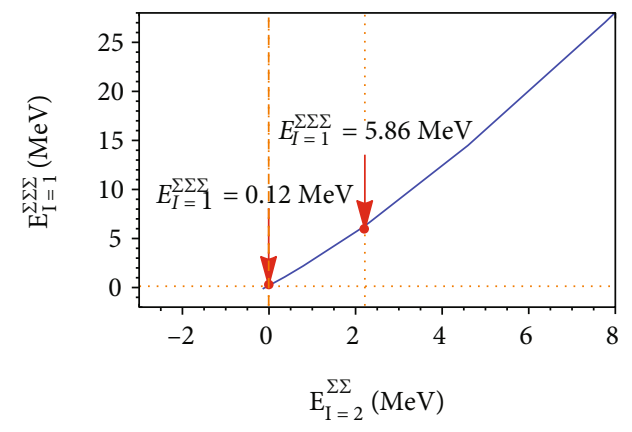

(f)

Figure 10: (a-c) Plots of wave functions for the $\Sigma \Sigma \Sigma$ system in the states $|0,1\rangle_{I} \otimes|0,1 / 2\rangle_{S},|1,(0,1,2)\rangle_{I} \otimes|1,1 / 2(3 / 2)\rangle_{S}$ and $|2,(1,2,3)\rangle_{I}$ $\otimes|0,1 / 2\rangle_{S}$, respectively. The blue lines denote the wave functions for any two $\Sigma$ in the $\Sigma \Sigma \Sigma$ system. The red lines represent the wave functions for its subsystem $\Sigma \Sigma$. (d-f) Plots of the dependence of the total three-body binding energy on the two-body binding energy of the subsystem $\Sigma \Sigma$ for the states $|0,1\rangle_{I} \otimes|0,1 / 2\rangle_{S},|1,(0,1,2)\rangle_{I} \otimes|1,1 / 2(3 / 2)\rangle_{S}$ and $|2,(1,2,3)\rangle_{I} \otimes|0,1 / 2\rangle_{S}$, respectively. The left red point indicates the Critical Borromean state of the system. The right one is our numerical result when the two-body binding energy is chosen at $2.23 \mathrm{MeV}$.

the solutions we solved in the last section are approximate solutions. The binding energies we obtained may be less than the strict values of the three-body systems.

However, we have admitted that the application of the method adopted in this work is sometimes limited. We have not consider the channel couplings of the three-body systems during our calculations. For instance, $\Lambda \Lambda \Lambda$ can couple with the $\Lambda N \Xi$ via mesons with strangeness exchange such as
$K$ and $K^{*}$. The $\Lambda N \Xi$ channel may contribute to the formation of $\Lambda \Lambda \Lambda$ three-body bound states. However, in this work, we neglect these couple channel effects and only focus on the case that the three constituents are identical particles. This is the limitation of our work. Since the generalized identity principle constricts the total wavefunctions which contain the space, isospin and spin wave functions should be antisymmetric. Then, only several possible isospin-spin 
configurations survive, which greatly simplify the calculations of the three-body system. The three-baryon system with strange mesons exchange will contain more complicated spin and isospin configurations, which will be carried out in a future work.

In short summary, we have performed an extensive investigation on the tritonlike system with three identical hadrons of baryon octet. i.e., the $N N N, \Lambda \Lambda \Lambda, \Xi \Xi \Xi$, and $\Sigma \Sigma \Sigma$. Except the $\Lambda \Lambda \Lambda$, all of the tritonlike systems have Critical Borromean states. It indicates that the $N N N, \Xi \Xi \Xi$, and $\Sigma \Sigma \Sigma$ systems could be bound states as long as their corresponding twobody subsystems have binding solutions. The efforts in this work might be helpful to understand the three-body systems in future. Hopefully, the future well-developed experiments on hadron collisions will provide us a platform to seek out the trihyperon molecules $\Xi \Xi \Xi$ and $\Sigma \Sigma \Sigma$.

\section{Appendix}

\section{A. The Matrix Element of the $\mathscr{H}_{a a} \mathscr{H}_{a a}$}

The matrix element of the $\mathscr{H}_{a a}$ has the form

$$
\begin{aligned}
\mathscr{H}_{a a}^{i j}= & \left\langle\tilde{\psi}_{a}^{i}|\mathscr{H}| \tilde{\psi}_{a}^{j}\right\rangle \\
= & \left\langle\frac{1}{N_{i}}\left[\left(\psi_{a}^{i}+\psi_{b}^{i}+\psi_{c}^{i}\right)-\sum_{i} x_{i m} \psi_{a}^{m}\right]|\mathscr{H}| \frac{1}{N_{j}}\right. \\
& \left.\cdot\left[\left(\psi_{a}^{j}+\psi_{b}^{j}+\psi_{c}^{j}\right)-\sum_{i} x_{j n} \psi_{a}^{n}\right]\right\rangle \\
= & 3 \frac{1}{N_{i} N_{j}}\left\langle\psi_{a}^{i}|\mathscr{H}| \psi_{a}^{j}\right\rangle+6 \frac{1}{N_{i} N_{j}}\left\langle\psi_{b}^{i}|\mathscr{H}| \psi_{a}^{j}\right\rangle \\
& -x_{i m} \frac{1}{N_{i} N_{j}}\left\langle\psi_{a}^{m}|\mathscr{H}| \psi_{a}^{j}\right\rangle-x_{j n} \frac{1}{N_{i} N_{j}}\left\langle\psi_{a}^{i}|\mathscr{H}| \psi_{a}^{n}\right\rangle \\
& -2 x_{i m} \frac{1}{N_{i} N_{j}}\left\langle\psi_{b}^{m}|\mathscr{H}| \psi_{a}^{j}\right\rangle-2 x_{j n} \frac{1}{N_{i} N_{j}}\left\langle\psi_{b}^{i}|\mathscr{H}| \psi_{a}^{n}\right\rangle \\
& +x_{i m} x_{j n} \frac{1}{N_{i} N_{j}}\left\langle\psi_{a}^{m}|\mathscr{H}| \psi_{a}^{n}\right\rangle,
\end{aligned}
$$

where the interchange symmetry in the $\mho \mho \mho$ system is used in the last step. Similarly, we have

$$
\begin{aligned}
\mathscr{H}_{b a}^{i j}= & \left\langle\tilde{\psi}_{b}^{i}|\mathscr{H}| \tilde{\psi}_{a}^{j}\right\rangle \\
= & \left\langle\frac{1}{N_{i}}\left[\left(\psi_{a}^{i}+\psi_{b}^{i}+\psi_{c}^{i}\right)-\sum_{i} x_{i m} \psi_{b}^{m}\right]|\mathscr{H}| \frac{1}{N_{j}}\right. \\
& \left.\cdot\left[\left(\psi_{a}^{j}+\psi_{b}^{j}+\psi_{c}^{j}\right)-\sum_{i} x_{j n} \psi_{a}^{n}\right]\right\rangle \\
= & 3 \frac{1}{N_{i} N_{j}}\left\langle\psi_{a}^{i}|\mathscr{H}| \psi_{a}^{j}\right\rangle+6 \frac{1}{N_{i} N_{j}}\left\langle\psi_{b}^{i}|\mathscr{H}| \psi_{a}^{j}\right\rangle \\
& -x_{i m} \frac{1}{N_{i} N_{j}}\left\langle\psi_{a}^{m}|\mathscr{H}| \psi_{a}^{j}\right\rangle-x_{j n} \frac{1}{N_{i} N_{j}}\left\langle\psi_{a}^{i}|\mathscr{H}| \psi_{a}^{n}\right\rangle \\
& -2 x_{i m} \frac{1}{N_{i} N_{j}}\left\langle\psi_{b}^{m}|\mathscr{H}| \psi_{a}^{j}\right\rangle-2 x_{j n} \frac{1}{N_{i} N_{j}}\left\langle\psi_{b}^{i}|\mathscr{H}| \psi_{a}^{n}\right\rangle \\
& +x_{i m} x_{j n} \frac{1}{N_{i} N_{j}}\left\langle\psi_{b}^{m}|\mathscr{H}| \psi_{a}^{n}\right\rangle .
\end{aligned}
$$

Since the interchange symmetry in the $\mho \mho \mho \mho$ system, only two matrices in Equation (21) are independent, which have the form

$$
\begin{aligned}
\left\langle\psi_{a}^{i}|\mathscr{H}| \psi_{a}^{j}\right\rangle= & |N|^{2} \int d \vec{r}_{b c}\left\{\left(2\left\langle\psi_{a b} \mid \psi_{a b}\right\rangle+\left\langle\psi_{a b} \mid \psi_{a c}\right\rangle\right)\right. \\
& \left.\cdot\left[\phi_{b c}^{i}\left(T_{*}^{\prime}+V_{B O}^{b c}\right) \phi_{b c}^{j}\right]+\left(\left\langle\psi_{a b} \mid \psi_{a c}\right\rangle\right)\left[\phi_{b c}^{i} V_{1}^{b c} \phi_{b c}^{j}\right]\right\} \\
= & \int d \vec{r}_{b c}\left\{\phi_{b c}^{i}\left(T_{*}^{\prime}+V_{B O}^{b c}\right) \phi_{b c}^{j}\right. \\
& \left.+\left(1-\frac{2}{2+\left\langle\psi_{a b} \mid \psi_{a c}\right\rangle}\right) \phi_{b c}^{i} V^{b c} \phi_{b c}^{j}\right\}, \\
\left\langle\psi_{b}^{i}|\mathscr{H}| \psi_{a}^{j}\right\rangle= & \frac{|N|^{2}}{2} \int d \vec{r}_{b c}\left\{\left\langle\psi_{a b} \phi_{a c}^{i}\left|T_{*}^{\prime}+V_{B O}^{b c}\right| \phi_{b c}^{j}\left(\psi_{a b}+\psi_{a c}\right)\right\rangle\right. \\
& +\left\langle\left(\psi_{a b}+\psi_{b c}\right) \phi_{a c}^{i}\left|T_{*}^{\prime}+V_{B O}^{b c}\right| \phi_{b c}^{j} \psi_{a b}\right\rangle \\
& +\left\langle\psi_{b c} \phi_{a c}^{i}\left|T_{*}^{\prime}+V_{B O}^{b c}\right| \phi_{b c}^{j} \psi_{a c}\right\rangle \\
& +\left\langle\psi_{b c} \phi_{a c}^{i}\left|V^{b c}\right| \phi_{b c}^{j} \psi_{a b}\right\rangle \\
& \left.+\left\langle\left(\psi_{a b}+\psi_{b c}\right) \phi_{a c}^{i}\left|V^{b c}\right| \phi_{b c}^{j} \psi_{a c}\right\rangle\right\} \\
= & \frac{1}{1+1 / 2\left\langle\psi_{a b} \mid \psi_{a c}\right\rangle}\left\{2\left\langle\psi_{a b} \phi_{a c}^{i}\left|T_{*}^{\prime}+V_{B O}^{b c}\right| \psi_{b c}^{j} \psi_{a b}\right\rangle\right. \\
& +\left\langle\psi_{a b} \phi_{a c}^{i}\left|T_{*}^{\prime}+V_{B O}^{b c}\right| \phi_{b c}^{j} \psi_{a c}\right\rangle+\left\langle\psi_{b c} \phi_{a c}^{i}\left|T_{*}^{\prime}+V_{B O}^{b c}\right| \psi_{b c}^{j} \psi_{a b}\right\rangle \\
& +\left\langle\psi_{b c} \phi_{a c}^{i}\left|T_{*}^{\prime}+V_{B O}^{b c}\right| \phi_{b c}^{j} \psi_{a c}\right\rangle+\left\langle\psi_{b c} \phi_{a c}^{i}\left|V^{b c}\right| \phi_{b c}^{j} \psi_{a b}\right\rangle \\
& \left.+\left\langle\psi_{a b} \phi_{a c}^{i}\left|V^{b c}\right| \phi_{b c}^{j} \psi_{a c}\right\rangle+\left\langle\psi_{b c} \phi_{a c}^{i}\left|V^{b c}\right| \phi_{b c}^{j} \psi_{a c}\right\rangle\right\},
\end{aligned}
$$

where we have introduced the abbreviations $\phi_{a b}^{i}, \phi_{b c}^{i}, \phi_{a c}^{i}$, $\psi_{a b}, \psi_{b c}, \psi_{a c}$ for $\phi\left(\vec{r}_{a b}\right)^{i}, \phi\left(\vec{r}_{b c}\right)^{i}, \phi\left(\vec{r}_{a c}\right)^{i}, \psi\left(\vec{r}_{a b}\right), \psi\left(\vec{r}_{b c}\right)$, and $\psi\left(\vec{r}_{a c}\right)$, respectively. The expression for the $\mathscr{H}_{c a}$ can be derived by the replacement $c \longrightarrow b, b \longrightarrow c$ on the expression for the $\mathscr{H}_{b a}$. Similarly, the expression for the $\mathscr{H}_{c b}$ is derived by the replacement $c \longrightarrow b, b \longrightarrow a, a \longrightarrow c$ on the expression for the $\mathscr{H}_{b a}$. The interchange invariance of the $\mho \mho \mho$ system is helpful to simplify the calculation, i.e., $\mathscr{H}_{c a}$ $=\mathscr{H}_{c b}=\mathscr{H}_{b a}$ and $\mathscr{H}_{a a}=\mathscr{H}_{b b}=\mathscr{H}_{c c}$.

\section{B. The Isospin Wave Functions of the Relevant Systems}

We define the isospin wave function of a tritonlike system as $\left|I_{2}, I_{3}, I_{3 z}\right\rangle$, where the $I_{2}$ is the isospin of its two-body subsystem and the $I_{3}$ and $I_{3 z}$ denote the total isospin of the threebody system and its $z$ direction, respectively. The isospin wave functions of the NNN system read

$$
\begin{aligned}
& \left|1, \frac{3}{2}, \frac{3}{2}\right\rangle=(p p) p, \\
& \left|1, \frac{3}{2}, \frac{1}{2}\right\rangle=\frac{1}{\sqrt{3}}[(n p) p+(p n) p+(p p) n], \\
& \left|1, \frac{3}{2},-\frac{1}{2}\right\rangle=\frac{1}{\sqrt{3}}[(n n) p+(n p) n+(p n) n],
\end{aligned}
$$




$$
\begin{aligned}
\left|1, \frac{3}{2},-\frac{3}{2}\right\rangle & =(n n) n, \\
\left|1, \frac{1}{2}, \frac{1}{2}\right\rangle & =\frac{1}{\sqrt{6}}[2(p p) n-(p n) p-(n p) p], \\
\left|1, \frac{1}{2},-\frac{1}{2}\right\rangle & =\frac{1}{\sqrt{6}}[(p n) n+(n p) n-2(n n) p], \\
\left|0, \frac{1}{2}, \frac{1}{2}\right\rangle & =\frac{1}{\sqrt{2}}[|(p n) p\rangle-|(n p) p\rangle], \\
\left|0, \frac{1}{2},-\frac{1}{2}\right\rangle & =\frac{1}{\sqrt{2}}[|(p n) n\rangle-|(n p) n\rangle] .
\end{aligned}
$$

The isospin wave functions of the $\Xi \Xi \Xi$ system read

$$
\begin{aligned}
& \left|1, \frac{3}{2}, \frac{3}{2}\right\rangle=\left(\Xi^{0} \Xi^{0}\right) \Xi^{0}, \\
& \left|1, \frac{3}{2}, \frac{1}{2}\right\rangle=\frac{1}{\sqrt{3}}\left[\left(\Xi^{-} \Xi^{0}\right) \Xi^{0}+\left(\Xi^{0} \Xi^{-}\right) \Xi^{0}+\left(\Xi^{0} \Xi^{0}\right) \Xi^{-}\right] \\
& \left|1, \frac{3}{2},-\frac{1}{2}\right\rangle=\frac{1}{\sqrt{3}}\left[\left(\Xi^{-} \Xi^{-}\right) \Xi^{0}+\left(\Xi^{-} \Xi^{0}\right) \Xi^{-}+\left(\Xi^{0} \Xi^{-}\right) \Xi^{-}\right] \\
& \left|1, \frac{3}{2},-\frac{3}{2}\right\rangle=\left(\Xi^{-} \Xi^{-}\right) \Xi^{-}, \\
& \left|1, \frac{1}{2}, \frac{1}{2}\right\rangle=\frac{1}{\sqrt{6}}\left[2\left(\Xi^{0} \Xi^{0}\right) \Xi^{-}-\left(\Xi^{0} \Xi^{-}\right) \Xi^{0}-\left(\Xi^{-} \Xi^{0}\right) \Xi^{0}\right], \\
& \left|1, \frac{1}{2},-\frac{1}{2}\right\rangle=\frac{1}{\sqrt{6}}\left[\left(\Xi^{0} \Xi^{-}\right) \Xi^{-}+\left(\Xi^{-} \Xi^{0}\right) \Xi^{-}-2\left(\Xi^{-} \Xi^{-}\right) \Xi^{0}\right] \\
& \left|0, \frac{1}{2}, \frac{1}{2}\right\rangle=\frac{1}{\sqrt{2}}\left[\left|\left(\Xi^{0} \Xi^{-}\right) \Xi^{0}\right\rangle-\left|\left(\Xi^{-} \Xi^{0}\right) \Xi^{0}\right\rangle\right] \\
& \left|0, \frac{1}{2},-\frac{1}{2}\right\rangle=\frac{1}{\sqrt{2}}\left[\left|\left(\Xi^{0} \Xi^{-}\right) \Xi^{-}\right\rangle-\left|\left(\Xi^{-} \Xi^{0}\right) \Xi^{-}\right\rangle\right] .
\end{aligned}
$$

The isospin wave functions of the $\Sigma \Sigma \Sigma$ system read

$$
\begin{aligned}
|2,3,3\rangle= & \left(\Sigma^{+} \Sigma^{+}\right) \Sigma^{+} \\
|2,3,2\rangle= & \frac{1}{\sqrt{3}}\left[\left(\Sigma^{+} \Sigma^{+}\right) \Sigma^{0}+\left(\Sigma^{+} \Sigma^{0}\right) \Sigma^{+}+\left(\Sigma^{0} \Sigma^{+}\right) \Sigma^{+}\right] \\
|2,3,1\rangle= & \frac{1}{\sqrt{15}}\left[\left(\Sigma^{+} \Sigma^{+}\right) \Sigma^{-}+2\left(\Sigma^{+} \Sigma^{0}\right) \Sigma^{0}+2\left(\Sigma^{0} \Sigma^{+}\right) \Sigma^{0}\right. \\
& \left.+\left(\Sigma^{+} \Sigma^{-}\right) \Sigma^{+}+2\left(\Sigma^{0} \Sigma^{0}\right) \Sigma^{+}+\left(\Sigma^{-} \Sigma^{+}\right) \Sigma^{+}\right] \\
|2,3,0\rangle= & \frac{1}{\sqrt{10}}\left[\left(\Sigma^{+} \Sigma^{0}\right) \Sigma^{-}+\left(\Sigma^{0} \Sigma^{+}\right) \Sigma^{-}\right. \\
& +\left(\Sigma^{+} \Sigma^{-}\right) \Sigma^{0}+2\left(\Sigma^{0} \Sigma^{0}\right) \Sigma^{0}+\left(\Sigma^{-} \Sigma^{+}\right) \Sigma^{0} \\
& \left.+\left(\Sigma^{0} \Sigma^{-}\right) \Sigma^{+}+\left(\Sigma^{-} \Sigma^{0}\right) \Sigma^{+}\right]
\end{aligned}
$$

$$
\begin{aligned}
|2,3,-1\rangle= & \frac{1}{\sqrt{15}}\left[\left(\Sigma^{+} \Sigma^{-}\right) \Sigma^{-}+2\left(\Sigma^{0} \Sigma^{0}\right) \Sigma^{-}+\left(\Sigma^{-} \Sigma^{+}\right) \Sigma^{-}\right. \\
& \left.+2\left(\Sigma^{0} \Sigma^{-}\right) \Sigma^{0}+2\left(\Sigma^{-} \Sigma^{0}\right) \Sigma^{0}+\left(\Sigma^{-} \Sigma^{-}\right) \Sigma^{+}\right], \\
|2,3,-2\rangle= & \frac{1}{\sqrt{3}}\left[\left(\Sigma^{0} \Sigma^{-}\right) \Sigma^{-}+\left(\Sigma^{-} \Sigma^{0}\right) \Sigma^{-}+\left(\Sigma^{-} \Sigma^{-}\right) \Sigma^{0}\right], \\
|2,3,-3\rangle= & \left(\Sigma^{-} \Sigma^{-}\right) \Sigma^{-}, \\
|2,2,2\rangle= & \frac{1}{\sqrt{6}}\left[\left(\Sigma^{+} \Sigma^{+}\right) \Sigma^{0}-\left(\Sigma^{+} \Sigma^{0}\right) \Sigma^{+}-\left(\Sigma^{0} \Sigma^{+}\right) \Sigma^{+}\right], \\
|2,2,1\rangle= & \frac{1}{2 \sqrt{3}}\left[2\left(\Sigma^{+} \Sigma^{+}\right) \Sigma^{-}+\left(\Sigma^{+} \Sigma^{0}\right) \Sigma^{0}+\left(\Sigma^{0} \Sigma^{+}\right) \Sigma^{0}\right. \\
& \left.-\left(\Sigma^{+} \Sigma^{-}\right) \Sigma^{+}-\left(\Sigma^{0} \Sigma^{0}\right) \Sigma^{+}-\left(\Sigma^{-} \Sigma^{+}\right) \Sigma^{+}\right],
\end{aligned}
$$$$
|2,2,0\rangle=\frac{1}{2}\left[\left(\Sigma^{+} \Sigma^{0}\right) \Sigma^{-}+\left(\Sigma^{0} \Sigma^{+}\right) \Sigma^{-}-\left(\Sigma^{0} \Sigma^{-}\right) \Sigma^{+}-\left(\Sigma^{-} \Sigma^{0}\right) \Sigma^{+}\right]
$$$$
|2,2,-1\rangle=\frac{1}{2 \sqrt{3}}\left[\left(\Sigma^{+} \Sigma^{-}\right) \Sigma^{-}+2\left(\Sigma^{0} \Sigma^{0}\right) \Sigma^{-}+\left(\Sigma^{-} \Sigma^{+}\right) \Sigma^{-}\right.
$$$$
\left.-\left(\Sigma^{0} \Sigma^{-}\right) \Sigma^{0}-\left(\Sigma^{-} \Sigma^{0}\right) \Sigma^{0}-2\left(\Sigma^{-} \Sigma^{-}\right) \Sigma^{+}\right],
$$$$
|2,2,-2\rangle=\frac{1}{\sqrt{6}}\left[\left(\Sigma^{0} \Sigma^{-}\right) \Sigma^{-}+\left(\Sigma^{-} \Sigma^{0}\right) \Sigma^{-}-2\left(\Sigma^{-} \Sigma^{-}\right) \Sigma^{0}\right],
$$$$
|2,1,1\rangle=\frac{\sqrt{15}}{30}\left[6\left(\Sigma^{+} \Sigma^{+}\right) \Sigma^{-}-3\left(\Sigma^{+} \Sigma^{0}\right) \Sigma^{0}-3\left(\Sigma^{0} \Sigma^{+}\right) \Sigma^{0}\right.
$$$$
\left.+\left(\Sigma^{+} \Sigma^{-}\right) \Sigma^{+}+2\left(\Sigma^{0} \Sigma^{0}\right) \Sigma^{+}+\left(\Sigma^{-} \Sigma^{+}\right) \Sigma^{+}\right] \text {, }
$$$$
|2,1,0\rangle=\frac{1}{2 \sqrt{15}}\left[3\left(\Sigma^{+} \Sigma^{0}\right) \Sigma^{-}+3\left(\Sigma^{0} \Sigma^{+}\right) \Sigma^{-}-2\left(\Sigma^{+} \Sigma^{-}\right) \Sigma^{0}\right.
$$$$
\left.-4\left(\Sigma^{0} \Sigma^{0}\right) \Sigma^{0}-2\left(\Sigma^{-} \Sigma^{+}\right) \Sigma^{0}+3\left(\Sigma^{0} \Sigma^{-}\right) \Sigma^{+} 3\left(\Sigma^{-} \Sigma^{0}\right) \Sigma^{+}\right],
$$$$
|2,1,-1\rangle=\frac{1}{2 \sqrt{15}}\left[\left(\Sigma^{+} \Sigma^{-}\right) \Sigma^{-}+2\left(\Sigma^{0} \Sigma^{0}\right) \Sigma^{-}+\left(\Sigma^{-} \Sigma^{+}\right) \Sigma^{-}\right.
$$$$
\left.-3\left(\Sigma^{0} \Sigma^{-}\right) \Sigma^{0}-3\left(\Sigma^{-} \Sigma^{0}\right) \Sigma^{0}+6\left(\Sigma^{-} \Sigma^{-}\right) \Sigma^{+}\right] \text {, }
$$$$
|1,2,2\rangle=\frac{1}{\sqrt{2}}\left[\left(\Sigma^{+} \Sigma^{0}\right) \Sigma^{+}-\left(\Sigma^{0} \Sigma^{+}\right) \Sigma^{+}\right]
$$$$
|1,2,1\rangle=\frac{1}{2}\left[\left(\Sigma^{+} \Sigma^{0}\right) \Sigma^{0}-\left(\Sigma^{0} \Sigma^{+}\right) \Sigma^{0}+\left(\Sigma^{+} \Sigma^{-}\right) \Sigma^{+}-\left(\Sigma^{-} \Sigma^{+}\right) \Sigma^{+}\right],
$$$$
|1,2,0\rangle=\frac{1}{2 \sqrt{3}}\left[\left(\Sigma^{+} \Sigma^{0}\right) \Sigma^{-}-\left(\Sigma^{0} \Sigma^{+}\right) \Sigma^{-}+2\left(\Sigma^{+} \Sigma^{-}\right) \Sigma^{0}\right.
$$$$
\left.-2\left(\Sigma^{-} \Sigma^{+}\right) \Sigma^{0}+\left(\Sigma^{0} \Sigma^{-}\right) \Sigma^{+}-\left(\Sigma^{-} \Sigma^{0}\right) \Sigma^{+}\right],
$$

$|1,2,-1\rangle=\frac{1}{2}\left[\left(\Sigma^{+} \Sigma^{-}\right) \Sigma^{-}+\left(\Sigma^{0} \Sigma^{-}\right) \Sigma^{0}-\left(\Sigma^{-} \Sigma^{+}\right) \Sigma^{-}-\left(\Sigma^{-} \Sigma^{0}\right) \Sigma^{0}\right]$,

$|1,2,-2\rangle=\frac{1}{\sqrt{2}}\left[\left(\Sigma^{0} \Sigma^{-}\right) \Sigma^{-}-\left(\Sigma^{-} \Sigma^{0}\right) \Sigma^{-}\right]$

$|1,1,1\rangle=\frac{1}{2}\left[\left(\Sigma^{+} \Sigma^{0}\right) \Sigma^{0}-\left(\Sigma^{0} \Sigma^{+}\right) \Sigma^{0}-\left(\Sigma^{+} \Sigma^{-}\right) \Sigma^{+}+\left(\Sigma^{-} \Sigma^{+}\right) \Sigma^{+}\right]$,

$|1,1,0\rangle=\frac{1}{2}\left[\left(\Sigma^{+} \Sigma^{0}\right) \Sigma^{-}+\left(\Sigma^{-} \Sigma^{0}\right) \Sigma^{+}-\left(\Sigma^{0} \Sigma^{+}\right) \Sigma^{-}-\left(\Sigma^{0} \Sigma^{-}\right) \Sigma^{+}\right]$, 


$$
\begin{aligned}
|1,1,-1\rangle= & \frac{1}{2}\left[\left(\Sigma^{+} \Sigma^{-}\right) \Sigma^{-}-\left(\Sigma^{-} \Sigma^{+}\right) \Sigma^{-}-\left(\Sigma^{0} \Sigma^{-}\right) \Sigma^{0}+\left(\Sigma^{-} \Sigma^{0}\right) \Sigma^{0}\right] \\
|1,0,0\rangle= & \frac{1}{\sqrt{6}}\left[\left(\Sigma^{+} \Sigma^{0}\right) \Sigma^{-}-\left(\Sigma^{0} \Sigma^{+}\right) \Sigma^{-}-\left(\Sigma^{+} \Sigma^{-}\right) \Sigma^{0}\right. \\
& \left.+\left(\Sigma^{-} \Sigma^{+}\right) \Sigma^{0}+\left(\Sigma^{0} \Sigma^{-}\right) \Sigma^{+}-\left(\Sigma^{-} \Sigma^{0}\right) \Sigma^{+}\right] .
\end{aligned}
$$

\section{The Functions $f_{1}, f_{2}, f_{3}, f_{4}, f_{5}$, and $f_{6}$}

The functions $f_{1}, f_{2}, f_{3}, f_{4}, f_{5}$, and $f_{6}$ in Equations (35)-(40) are defined as

$$
\begin{aligned}
& f_{1}\left(m_{\alpha}, \lambda, r, \vec{\sigma}_{1} \cdot \vec{\sigma}_{2}\right) \\
& =\frac{1}{3} \vec{\sigma}_{1} \cdot \vec{\sigma}_{2}\left\{m_{\alpha}^{2} \lambda Y(\lambda r)-m_{\alpha}^{3} Y\left(m_{\alpha} r\right)+\left(\lambda^{2}-m_{\alpha}^{2}\right) \lambda \frac{1}{2} e^{-\lambda r}\right\}, \\
& f_{2}\left(m_{\alpha}, \lambda, r, S_{12}\right) \\
& =\frac{1}{3} S_{12}(\vec{r})\left\{-m_{\alpha}^{3} Z\left(m_{\alpha} r\right)+\lambda^{3} Z(\lambda r)+\left(\lambda^{2}-m_{\alpha}^{2}\right)(1+\lambda r) \frac{\lambda}{2} Y(\lambda r)\right\}, \\
& f_{3}\left(m_{\alpha}, \lambda, r\right) \\
& \quad=m_{\alpha} Y\left(m_{\alpha} r\right)-\lambda Y(\lambda r)-\left(\lambda^{2}-m_{\alpha}^{2}\right) \frac{1}{2 \lambda} e^{-\lambda r}, \\
& f_{4}\left(m_{\alpha}, \lambda, r\right) \\
& \quad=m_{\alpha}^{2} \lambda Y(\lambda r)-m_{\alpha}^{3} Y\left(m_{\alpha} r\right)+\left(\lambda^{2}-m_{\alpha}^{2}\right) \lambda \frac{1}{2} e^{-\lambda r}, \\
& f_{6}\left(m_{\alpha}, \lambda, r\right) \\
& =m_{\alpha}^{3} Y\left(m_{\alpha} r\right)-\lambda^{3} Y(\lambda r)+\left(\lambda^{2}-m_{\alpha}^{2}\right)\left(1-\frac{1}{2} \lambda r\right) \lambda Y(\lambda r), \\
& f_{5}\left(m_{\alpha}, \lambda, r, \vec{L} \cdot \vec{S}\right) \\
& =\vec{L} \cdot \vec{S}\left\{-m_{\alpha}^{3} Z_{1}\left(m_{\alpha} r\right)+\lambda^{3} Z_{1}(\lambda r)+\left(\lambda^{2}-m_{\alpha}^{2}\right) \frac{1}{2 r} e^{-\lambda r}\right\},
\end{aligned}
$$

where $\alpha=\pi, \eta, \sigma, \rho, \omega, \phi$. The functions $Y, Z$, and $Z_{1}$ in the above are defined as

$$
\begin{aligned}
& Y\left(m_{\alpha} r\right)=\frac{\exp \left(m_{\alpha} r\right)}{m_{\alpha} r} \\
& Z\left(m_{\alpha} r\right)=\left(1+\frac{3}{m_{\alpha} r}+\frac{3}{\left(m_{\alpha} r\right)^{2}}\right) Y\left(m_{\alpha} r\right), \\
& Z_{1}\left(m_{\alpha}\right)=\left(\frac{1}{m_{\alpha} r}+\frac{1}{\left(m_{\alpha} r\right)^{2}}\right) Y\left(m_{\alpha} r\right) .
\end{aligned}
$$

\section{The Integrals $\left\langle\psi_{a b} \mid \psi_{a c}\right\rangle,\left\langle\psi_{a b}\left|\widehat{O}_{a b}\right| \psi_{a c}\right\rangle$, and $\left\langle\psi_{a b}\left|\widehat{O}_{a c}\right| \psi_{a c}\right\rangle$}

The cross integrals in Equation (8) can be performed in ellipsoidal coordinates. We set the long axis of ellipsoidal long with the direction of the $\vec{r}_{b c}$, and then, we have the coordinate transformation

$$
\left\{\begin{array}{l}
\zeta=\frac{1}{R}\left(r_{a b}+r_{a c}\right) \\
\kappa=\frac{1}{R}\left(r_{a b}-r_{a c}\right) \\
\varphi=\varphi_{a b}=\varphi_{a c} .
\end{array}\right.
$$

Here, we put the long axis of ellipsoidal as $z$-axis. The polar angles of the $\vec{r}_{a b}$ and $\vec{r}_{a c}$ can be expressed in ellipsoidal coordinates as

$$
\left\{\begin{array}{l}
\cos \theta_{a b}=\frac{1+\zeta \kappa}{\zeta+\kappa} \\
\cos \theta_{a c}=\frac{1-\zeta \kappa}{\zeta-\kappa}
\end{array}\right.
$$

The volume element in ellipsoidal coordinates is

$$
d \tau=\frac{R^{3}}{8}\left(\zeta^{2}-\kappa^{2}\right) d \zeta d \kappa d \varphi
$$

The Laplace operator in ellipsoidal coordinates has the form

$$
\begin{aligned}
\nabla^{2}= & \frac{4}{R^{2}\left(\zeta^{2}-\kappa^{2}\right)}\left\{\frac{\partial}{\partial \zeta}\left[\left(\zeta^{2}-1\right) \frac{\partial}{\partial \zeta}\right]\right. \\
& \left.+\frac{\partial}{\partial \kappa}\left[\left(1-\kappa^{2}\right) \frac{\partial}{\partial \kappa}\right]+\frac{\left(\zeta^{2}-\kappa^{2}\right)}{\left(\zeta^{2}-1\right)\left(1-\kappa^{2}\right)} \frac{\partial^{2}}{\partial \varphi^{2}}\right\}
\end{aligned}
$$

By using Laguerre polynomials, we expand the wave functions $\psi\left(\vec{r}_{a b}\right)$ and $\psi\left(\vec{r}_{a c}\right)$ as

$$
\left\{\begin{array}{l}
\psi\left(\vec{r}_{a b}\right)=\sum_{n=0}^{N-1} a_{n} \chi_{n l}\left(r_{a b}\right) \phi_{l}\left(\theta_{a b}, \varphi_{a b}\right), \\
\psi\left(\vec{r}_{a c}\right)=\sum_{n^{\prime}=0}^{N^{\prime}-1} b_{n^{\prime}} \chi_{n^{\prime} l^{\prime}}\left(r_{a c}\right) \phi_{l^{\prime}}\left(\theta_{a c}, \varphi_{a c}\right),
\end{array}\right.
$$

where the $\phi_{l}$ and $\phi_{l^{\prime}}$ are the angle parts of the wave functions. In general, the $l\left(l^{\prime}\right)=0,1,2 \cdots$, which corresponds to the case of $\mathrm{S}$ wave, $\mathrm{P}$ wave, and $\mathrm{D}$ wave. Thus, the cross integrals of the type $\left\langle\psi_{a b}\left|\widehat{O}_{a b}\right| \psi_{a c}\right\rangle$ read 


$$
\begin{aligned}
\left\langle\psi_{a b}\left|\widehat{O}_{a b}\right| \psi_{a c}\right\rangle= & \sum_{n=0}^{N-1} \sum_{n}^{\prime=0} N^{\prime-1} a_{n} b_{n^{\prime}}\left\langle\chi_{n 1}\left(r_{a b}\right) Y_{l m}\left(\theta_{a b}, \varphi_{a b}\right)\right. \\
& \left.\cdot\left|\widehat{O}\left(r_{a b}, \theta_{a b}, \varphi_{a b}\right)\right| \chi_{n^{\prime} l^{\prime}}\left(r_{a c}\right) Y_{l^{\prime} m^{\prime}}\left(\theta_{a c}, \varphi_{a c}\right)\right\rangle \\
= & \sum_{n, n^{\prime}} a_{n} b_{n^{\prime}}\left[\frac{n !(2 \xi)^{2 l+3}}{\Gamma(2 l+3+n)}\right]^{1 / 2}\left[\frac{n^{\prime} !\left(2 \xi^{\prime}\right)^{2 l^{\prime}+3}}{\Gamma\left(2 l^{\prime}+3+n^{\prime}\right)}\right]^{1 / 2} \\
& \cdot\left[\frac{(2 l+1)(l-m) !}{2(l+m) !}\right]^{1 / 2}\left[\frac{\left(2 l^{\prime}+1\right)\left(l^{\prime}-m^{\prime}\right) !}{2\left(l^{\prime}+m^{\prime}\right) !}\right]^{1 / 2} \\
& \times \frac{R^{3}}{8} \iint d \zeta d \kappa\left(\zeta^{2}-\kappa^{2}\right) \\
& \cdot\left[\left(\frac{R}{2}(\zeta+\kappa)\right)^{l} e^{-\xi R / 2(\zeta+\kappa)} L_{n}^{2 l+2}(\xi R(\zeta+\kappa)) P_{l}^{m}\left(\frac{1+\zeta \kappa}{\zeta+\kappa}\right)\right] \\
& \times \widehat{O}\left(\frac{R}{2}(\zeta+\kappa), \arccos \left(\frac{1+\zeta \kappa}{\zeta+\kappa}\right), \varphi\right) \\
& \cdot\left[\left(\frac{R}{2}(\zeta-\kappa)\right)^{l^{\prime}} e^{-\xi^{\prime} R / 2(\zeta-\kappa)} L_{n^{\prime}}^{2 l^{\prime}+2}\left(\xi^{\prime} R(\zeta-\kappa)\right) P_{l^{\prime}}^{m^{\prime}}\left(\frac{1-\zeta \kappa}{\zeta-\kappa}\right)\right],
\end{aligned}
$$

where the operator $\widehat{O}_{a b}$ could be Laplace operator, effective potential, or unit operator. Similarly, the cross integrals of the type $\left\langle\psi_{a b}\left|\widehat{O}_{a c}\right| \psi_{a c}\right\rangle$ read

$$
\begin{aligned}
\left\langle\psi_{a b}\left|\widehat{O}_{a c}\right| \psi_{a c}\right\rangle= & \sum_{n=0}^{N-1} \sum_{n^{\prime}=0}^{N^{\prime}-1} a_{n} b_{n^{\prime}}\left\langle\chi_{n l}\left(r_{a b}\right) Y_{l m}\left(\theta_{a b}, \varphi_{a b}\right)\right. \\
& \left.\cdot\left|\widehat{O}\left(r_{a c}, \theta_{a c}, \varphi_{a c}\right)\right| \chi_{n^{\prime} l^{\prime}}\left(r_{a c}\right) Y_{l^{\prime} m^{\prime}}\left(\theta_{a c}, \varphi_{a c}\right)\right\rangle \\
= & \sum_{n, n^{\prime}} a_{n} b_{n^{\prime}}\left[\frac{n !(2 \xi)^{2 l+3}}{\Gamma(2 l+3+n)}\right]^{1 / 2}\left[\frac{n^{\prime} !\left(2 \xi^{\prime}\right)^{2 l^{\prime}+3}}{\Gamma\left(2 l^{\prime}+3+n^{\prime}\right)}\right]^{1 / 2} \\
& \cdot\left[\frac{(2 l+1)(l-m) !}{2(l+m) !}\right]^{1 / 2}\left[\frac{\left(2 l^{\prime}+1\right)\left(l^{\prime}-m^{\prime}\right) !}{2\left(l^{\prime}+m^{\prime}\right) !}\right]^{1 / 2} \\
& \times \frac{R^{3}}{8} \iint d \zeta d \kappa\left(\zeta^{2}-\kappa^{2}\right) \\
& \cdot\left[\left(\frac{R}{2}(\zeta+\kappa)\right)^{l} e^{-\xi R / 2(\zeta+\kappa)} L_{n}^{2 l+2}(\xi R(\zeta+\kappa)) P_{l}^{m}\left(\frac{1+\zeta \kappa}{\zeta+\kappa}\right)\right] \\
& \times \widehat{O}\left(\frac{R}{2}(\zeta-\kappa), \arccos \left(\frac{1-\zeta \kappa}{\zeta-\kappa}\right), \varphi\right) \\
& \cdot\left[\left(\frac{R}{2}(\zeta-\kappa)\right)^{l^{\prime}} e^{-\xi^{\prime} R / 2(\zeta-\kappa)} L_{n^{\prime}}^{2 l^{\prime}+2}\left(\xi^{\prime} R(\zeta-\kappa)\right) P_{l^{\prime}}^{m^{\prime}}\left(\frac{1-\zeta \kappa}{\zeta-\kappa}\right)\right] .
\end{aligned}
$$

If the $\widehat{O}_{a b}=\widehat{O}_{a c}=1$, we obtain the value of the integral $\left\langle\psi_{a b} \mid \psi_{a c}\right\rangle$.

\section{Data Availability}

The masses of $\pi, \eta, \sigma, \rho, \omega, \phi, N, \Lambda, \Xi$, and $\Sigma$ are taken from the PDG (doi:10.1103/PhysRevD.98.030001). By fitting to experimental data, we adopt the values $g_{\pi N N}=13.07, g_{\eta N N}$ $=2.24, g_{\sigma N N}=8.46, g_{\rho N N}=3.25, f_{\rho N N}=6.1 g_{\rho N N}, g_{\omega N N}=$ 15.85, and $f_{\omega N N}=0$ from Refs. doi:10.1103/PhysRevD.84 .014031, doi:10.1016/S0370-1573(87)80002-9, doi:10.1103/ PhysRevC.63.024001, and doi:10.1103/PhysRevC.81.065201.

\section{Disclosure}

A preprint has previously been published [92].

\section{Conflicts of Interest}

The author declares no conflicts of interest.

\section{Acknowledgments}

This work is supported in part by the Fundamental Research Funds for the Central Universities under Grant No. 2020RC005.

\section{References}

[1] H. X. Chen, W. Chen, X. Liu, and S. L. Zhu, "Vital nodes identification in complex networks," Physics Reports, vol. 650, pp. 1-63, 2016.

[2] H. X. Chen, W. Chen, X. Liu, Y. R. Liu, and S. L. Zhu, "A review of the open charm and open bottom systems," Reports on Progress in Physics, vol. 80, no. 7, article 076201, 2017.

[3] E. Oset, W. H. Liang, M. Bayar et al., "Weak decays of heavy hadrons into dynamically generated resonances," International Journal of Modern Physics E, vol. 25, no. 1, article 1630001, 2016.

[4] A. Esposito, A. Pilloni, and A. D. Polosa, "Multiquark resonances, Phys," 2017, http://arxiv.org/abs/1611.07920.

[5] R. F. Lebed, R. E. Mitchell, and E. S. Swanson, "Heavy-quark QCD exotica," Progress in Particle and Nuclear Physics, vol. 93, pp. 143-194, 2017.

[6] A. Hosaka, T. Iijima, K. Miyabayashi, Y. Sakai, and S. Yasui, "Exotic hadrons with heavy flavors: X, Y, Z, and related states," Progress of Theoretical and Experimental Physics, vol. 2016, no. 6, article $062 \mathrm{C} 01,2016$.

[7] S. L. Olsen, T. Skwarnicki, and D. Zieminska, "Nonstandard heavy mesons and baryons: experimental evidence," Reviews of Modern Physics, vol. 90, no. 1, article 015003, 2018.

[8] A. Francis, R. J. Hudspith, R. Lewis, and K. Maltman, "Lattice prediction for deeply bound doubly heavy tetraquarks," Physical Review Letters, vol. 118, no. 14, article 142001, 2017.

[9] M. Karliner and J. L. Rosner, "Discovery of the doubly charmed $\Xi c c$ baryon implies a stable bbu ${ }^{-} \mathrm{d}^{-}$tetraquark," Physical Review Letters, vol. 119, no. 20, article 202001, 2017.

[10] E. J. Eichten and C. Quigg, "Heavy-quark symmetry implies stable heavy tetraquark mesons Q i Q j q k q l," Physical Review Letters, vol. 119, no. 20, article 202002, 2017.

[11] S. L. Olsen, "A new hadron spectroscopy," AIP Conference Proceedings, vol. 1701, no. 1, 2016.

[12] F. K. Guo, C. Hanhart, U. G. Meißner, Q. Wang, Q. Zhao, and B. S. Zou, "Hadronic molecules," Reviews of Modern Physics, vol. 90, no. 1, 2018.

[13] Y. Dong, A. Faessler, and V. E. Lyubovitskij, "Description of heavy exotic resonances as molecular states using phenomenological Lagrangians," Progress in Particle and Nuclear Physics, vol. 94, pp. 282-310, 2017.

[14] Q. Wang, C. Hanhart, and Q. Zhao, "Decoding the riddle of Y (4260) and $Z_{c}(3900)$," Physical Review Letters, vol. 111, no. 13, article 132003, 2013. 
[15] H. X. Chen, W. Chen, X. Liu, T. G. Steele, and S. L. Zhu, "Towards exotic hidden-charm pentaquarks in QCD," Physical Review Letters, vol. 115, no. 17, article 172001, 2015.

[16] U. G. Meißner and J. A. Oller, "Testing the $\chi$ clp composite nature of the Pc (4450)," Physics Letters B, vol. 751, pp. 5962,2015

[17] C. J. Xiao, Y. Huang, Y. B. Dong, L. S. Geng, and D. Y. Chen, "Exploring the molecular scenario of P c (4312), $\mathrm{P}_{\mathrm{c}}$ (4440), and $\mathrm{P}_{\mathrm{c}}$ (4457)," Physical Review D, vol. 100, no. 1, article $014022,2019$.

[18] M. L. Du, V. Baru, F. K. Guo et al., "Interpretation of the LHCb $\mathrm{P}_{c}$ states as hadronic molecules and hints of a narrow $\mathrm{P}_{\mathrm{c}}$ " Physical Review Letters, vol. 124, no. 7, article 072001, 2020.

[19] J. He, X. Liu, Z. F. Sun, and S. L. Zhu, " $Z_{c}$ (4025) as the hadronic molecule with hidden charm," The European Physical Journal C, vol. 73, no. 11, 2013.

[20] F. L. Wang, R. Chen, Z. W. Liu, and X. Liu, "Possible triplecharm molecular pentaquarks from $\Xi_{c c} \mathrm{D}_{1} / \Xi_{c c} \mathrm{D} *_{2}$ interactions," Physical Review D, vol. 99, no. 5, article 054021, 2019.

[21] K. Chen, R. Chen, Z. F. Sun, and X. Liu, "K囚 molecular explanation to the newly observed $\Xi(1620)^{0}$," Physical Review D, vol. 100, no. 7, article 074006, 2019.

[22] N. Lee, Z. G. Luo, X. L. Chen, and S. L. Zhu, "Possible deuteronlike molecular states composed of heavy baryons," Physical Review D, vol. 84, no. 1, article 014031, 2011.

[23] R. Chen, X. Liu, X. Q. Li, and S. L. Zhu, "Identifying exotic hidden-charm pentaquarks," Physical Review Letters, vol. 115, no. 13, article 132002, 2015.

[24] L. Zhao, L. Ma, and S. L. Zhu, "The spin-orbit force, recoil corrections and possible $\mathrm{B} * \overline{\mathrm{B}} *$ and $\mathrm{D} * \overline{\mathrm{D}} *$ molecular states," 2014, http://arxiv.org/abs/1403.4043.

[25] L. Zhao, L. Ma, and S. L. Zhu, "The recoil correction and spinorbit force for the possible $\mathrm{B} * \overline{\mathrm{B}} *$ and $\mathrm{D} * \overline{\mathrm{D}} *$ states," 2015 , http://arxiv.org/abs/1504.04117.

[26] Z. F. Sun, J. He, X. Liu, Z. G. Luo, and S. L. Zhu, "Zb(10610) \pm and $\mathrm{Zb}(10650) \pm$ as the $\mathrm{B} * \mathrm{~B}^{-}$and $\mathrm{B} * \mathrm{~B}^{-} *$ molecular states," Physical Review D, vol. 84, no. 5, 2011.

[27] R. A. Malfliet and J. A. Tjon, "Solution of the Faddeev equations for the triton problem using local two-particle interactions," Nuclear Physics A, vol. 127, no. 1, pp. 161-168, 1969.

[28] G. Eichmann, R. Alkofer, A. Krassnigg, and D. Nicmorus, "Nucleon mass from a covariant three-quark Faddeev equation," Physical Review Letters, vol. 104, no. 20, article 201601, 2010.

[29] N. Ishii, W. Bentz, and K. Yazaki, "Baryons in the NJL model as solutions of the relativistic Faddeev equation," Nuclear Physics A, vol. 587, no. 4, pp. 617-656, 1995.

[30] G. Eichmann, "Nucleon electromagnetic form factors from the covariant Faddeev equation," Physical Review D, vol. 84, no. 1, article 014014, 2011.

[31] S. Huang and J. Tjon, "Nucleon solution of the Faddeev equation in the Nambu-Jona-Lasinio model," Physical Review C, vol. 49 , no. 3 , p. $1702,1994$.

[32] N. Ishii, W. Bentz, and K. Yazaki, "Faddeev approach to the nucleon in the Nambu-Jona-Lasinio (NJL) model," Physics Letters B, vol. 301, no. 2-3, pp. 165-169, 1993.

[33] S. Ishikawa, "Low-energy proton-deuteron scattering with a Coulomb-modified Faddeev equation," Few-Body Systems, vol. 32, no. 229, 2003.

[34] H. Sanchis-Alepuz, G. Eichmann, S. Villalba-Chavez, and R. Alkofer, "Delta and omega masses in a three-quark covari- ant Faddeev approach," Physical Review D, vol. 84, no. 9, article 096003, 2011.

[35] C. Elster, W. Glöckle, and H. Witala, "A new approach to the 3D Faddeev equation for three-body scattering," Few-Body Systems, vol. 45, no. 1, pp. 1-10, 2009.

[36] G. Eichmann, I. C. Cloet, R. Alkofer, A. Krassnigg, and C. D. Roberts, "Toward unifying the description of meson and baryon properties," Physical Review C, vol. 79, no. 1, article 012202, 2009.

[37] C. Popovici, P. Watson, and H. Reinhardt, "Three-quark confinement potential from the Faddeev equation," Physical Review D, vol. 83, no. 2, article 025013, 2011.

[38] Y. Fujiwara, M. Kohno, and Y. Suzuki, "Solving three-cluster OCM equations in the Faddeev formalism," Few-Body Systems, vol. 34, no. 4, pp. 237-257, 2004.

[39] A. Stadler, W. Glöckle, and P. U. Sauer, "Faddeev equations with three-nucleon force in momentum space," Physical Review C, vol. 44, no. 6, p. 2319, 1991.

[40] M. P. Valderrama, "Three-body $J^{P}=0^{+}, 1^{+}, 2^{+} B * B * \bar{K}$ bound states," Physical Review D, vol. 98, no. 1, article 014022, 2018.

[41] J. Révai and N. Shevchenko, "Faddeev calculations of the $\bar{K} N N$ system with a chirally motivated $\bar{K} N$ interaction. II. The K'pp quasibound state," Physical Review C, vol. 90, no. 3, article 034004, 2014.

[42] A. M. Torres, K. P. Khemchandani, L. S. Geng, M. Napsuciale, and E. Oset, "X (2175) as a resonant state of the $\phi \mathrm{K}$ Kmacr system," Physical Review D, vol. 78, no. 7, article 074031, 2008.

[43] A. M. Torres, K. P. Khemchandani, D. Jido, and A. Hosaka, "Theoretical support for the $\pi(1300)$ and the recently claimedf0(1790)as molecular resonances," Physical Review D, vol. 84, no. 7, article 074027, 2011.

[44] A. M. Torres, D. Jido, and Y. Kanada-En'yo, “Theoretical study of the $K K \bar{K}$ system and dynamical generation of the K (1460) resonance," Physical Review C, vol. 83, no. 6, article 065205, 2011.

[45] J. J. Xie, A. M. Torres, and E. Oset, "Studying $N *$ and $\Delta *$ states from $N \bar{K} K$ system with a three-body calculation," Physical Review Letters, vol. 83, article 065207, 2011.

[46] A. M. Torres, K. P. Khemchandani, D. Gamermann, and E. Oset, "Y (4260) as a J/Sigma KK system," Physical Review $D$, vol. 80, no. 9, article 094012, 2009.

[47] C. W. Xiao, M. Bayar, and E. Oset, "NDK,(K) over-barDN, and ND (K) over-bar molecules," Physical Review D, vol. 84, no. 3, article 034037, 2011.

[48] J. M. Dias, V. R. Debastiani, L. Roca, S. Sakai, and E. Oset, "Binding of the $B D \bar{D}$ and $B D D$ systems," Physical Review D, vol. 96, no. 9, article 094007, 2017.

[49] X. L. Ren, B. B. Malabarba, L. S. Geng, K. P. Khemchandani, and A. M. Torres, "K mesons with hidden charm arising from KX (3872) and KZc (3900) dynamics," Physics Letters B, vol. 785, pp. 112-117, 2018.

[50] H. Garcilazo and A. Valcarce, "Tbbb: a three B-meson bound state,” Physics Letters B, vol. 784, pp. 169-172, 2018.

[51] H. Garcilazo and A. Valcarce, "Deeply bound $\Xi$ tribaryon," Physical Review C, vol. 93, no. 3, article 034001, 2016.

[52] H. Garcilazo and A. Valcarce, " $\Omega N N$ and $\Omega \Omega N$ states," Physical Review C, vol. 99, no. 1, article 014001, 2019.

[53] D. Jido and Y. Kanada-En'yo, "KKN molecule state with $I=1 / 2$ and $J\{\sup P\}=1 / 2\{$ sup +$\}$ studied with a three-body calculation," Physical Review. C, Nuclear Physics, vol. 78, no. 3, 2008. 
[54] A. M. Torres, K. P. Khemchandani, and E. Oset, "Solution to Faddeev equations with two-body experimental amplitudes as input and application to $J^{P}=1 / 2^{+}, S=0$ baryon resonances," Physical Review C, vol. 79, no. 6, article 065207, 2009.

[55] M. Bayar and E. Oset, "Improved fixed center approximation of the Faddeev equations for the $\mathrm{K}^{-} \mathrm{NN}$ system with $\mathrm{S}=0$," Nuclear Physics A, vol. 883, pp. 57-68, 2012.

[56] E. Oset, D. Jido, T. Sekihara et al., "A new perspective on the Faddeev equations and the (K) over-bar NN system from chiral dynamics and unitarity in coupled channels," Nuclear Physics A, vol. 881, no. SI, pp. 127-140, 2012.

[57] A. M. Torres, E. J. Garzon, E. Oset, and L. R. Dai, "Limits to the fixed center approximation to Faddeev equations: the case of the $\phi$ (2170)," Physical Review D, vol. 83, no. 11, article 116002, 2011.

[58] W. Liang, C. W. Xiao, and E. Oset, "A study of $\eta K \bar{K}$ and $\eta K \bar{K}$ with the fixed center approximation to Faddeev equations," 2013, http://arxiv.org/abs/1309.7310.

[59] M. Bayar, X. L. Ren, and E. Oset, "States of $\rho D * \bar{D} *$ with $\mathrm{J}=3$ within the fixed center approximation to the Faddeev equations," The European Physical Journal A, vol. 51, no. 5, pp. 1-9, 2015.

[60] J. Yamagata-Sekihara, L. Roca, and E. Oset, "Nature of the $\mathrm{K} *_{2}$ (1430), $\mathrm{K} *_{3}(1780), \mathrm{K} *_{4}(2045), \mathrm{K} *_{5}(2380)$, and $\mathrm{K} *_{6}$ as $\mathrm{K} *-$ multi- $\rho$ states," Physical Review D, vol. 82, no. 9, article 094017, 2010.

[61] L. Roca and E. Oset, "Description of the $f(2)(1270)$, rho (3) (1690), f (4)(2050), rho (5)(2350), f (6)(2510) resonances as multi-rho (770) states," Physical Review Letters, vol. 82, article 054013, 2010.

[62] J. J. Xie, A. M. Torres, E. Oset, and P. Gonzalez, "Faddeev fixed-center approximation to the $\mathrm{N} \overline{\mathrm{K}} \mathrm{K}$ system and the signature of a N*(1920)(1/2+) state," Physical Review C, vol. 83, no. $6,2011$.

[63] V. R. Debastiani, J. M. Dias, and E. Oset, "Study of the DKK and DKK ," Physical Review D, vol. 96, no. 1, article 016014, 2017.

[64] C. Hajduk and P. U. Sauer, "Effect of the $\Delta$ (1236) isobar on the three-nucleon bound states," Nuclear Physics A, vol. 322, no. 23, pp. 329-348, 1979.

[65] Y. Ikeda and T. Sato, "Strange dibaryon resonance in theK ${ }^{-} \mathrm{NN}-\pi$ YNsystem," Physical Review $C$, vol. 76, no. 3, article 035203, 2007.

[66] Y. Ikeda and T. Sato, "Resonance energy of the $\bar{K} N N-\pi Y N$ system," Physical Review C, vol. 79, no. 3, article 035201, 2009.

[67] A. Gal and H. Garcilazo, "Three body calculation of the $\Delta \Delta$ dibaryon candidate D 03 (2370)," Physical Review Letters, vol. 111, no. 17, article 172301, 2013.

[68] K. Dreissigacker, S. Furui, C. Hajduk, P. U. Sauer, and R. Machleidt, "The mass of a bound $\Delta$-isobar," Nuclear Physics $A$, vol. 375, no. 3, pp. 334-360, 1982.

[69] S. König, H. W. Grießhammer, H. W. Hammer, U. van Kolck, and J. Phys, "Effective theory of $3 \mathrm{H}$ and $3 \mathrm{He}$," Journal of Physics G: Nuclear and Particle Physics, vol. 43, no. 5, article 055106, 2016.

[70] S. König and H. W. Hammer, "The proton-deuteron scattering length in pionless EFT," EPJ Web of Conferences, vol. 113, article 04011, 2016.

[71] E. Wilbring, H.-W. Hammer, and U.-G. Meißner, “Threebody universality in the B meson sector," http://arxiv.org/ abs/1705.06176.
[72] M. Schmidt, M. Jansen, and H.-W. Hammer, "Threshold effects and the line shape of the $X(3872)$ in effective field theory," Physical Review D, vol. 98, no. 1, article 014032, 2018.

[73] H. W. Hammer, J. Y. Pang, and A. Rusetsky, “Three-particle quantization condition in a finite volume: 1 . The role of the three-particle force," Journal of High Energy Physics, vol. 2017, no. 9, pp. 1-23, 2017.

[74] H.-W. Hammer, J.-Y. Pang, and A. Rusetsky, “Three-particle quantization condition in a finite volume: 2 . General formalism and the analysis of data," Journal of High Energy Physics, vol. 2017, no. 10, pp. 1-31, 2017.

[75] Y. Meng, C. Liu, U.-G. Meißner, and A. Rusetsky, “Three-particle bound states in a finite volume: unequal masses and higher partial waves," Physical Review D, vol. 98, no. 1, article 014508, 2018.

[76] E. Hiyama and M. Kamimura, "Study of various few-body systems using Gaussian expansion method (GEM)," Frontiers of Physics, vol. 13, no. 6, 2018.

[77] H. Kameyama, M. Kamimura, and Y. Fukushima, "Coupledrearrangement-channel Gaussian-basis variational method for trinucleon bound states," Physical Review Letters, vol. 40, pp. 974-987, 1989.

[78] T. W. Wu, M. Z. Liu, L. S. Geng, E. Hiyama, and M. P. Valderrama, " $D K, D D K$, and $D D D K$ molecules-understanding the nature of the $\mathrm{D} *_{\mathrm{s} 0}(2317)$," Physical Review D, vol. 100, no. 3, article 034029, 2019.

[79] R. Machleidt, K. Holinde, and C. Elster, "The meson theory of nuclear forces and nuclear structure," Advances in Nuclear Physics, vol. 149, 1987.

[80] M. M. Nagels, T. A. Rijken, and J. J. de Swart, "Baryon-baryon scattering in a one-boson-exchange-potential approach. II. Hyperon-nucleon scattering," Physical Review D, vol. 15, no. 9, pp. 2547-2564, 1977.

[81] M. M. Nagels, T. A. Rijken, and J. J. de Swart, "Low-energy nucleon-nucleon potential from Regge-pole theory," Physical Review D, vol. 17, no. 3, p. 768, 1978.

[82] P. M. M. Maessen, T. A. Rijken, and J. J. de Swart, "Soft-core baryon-baryon one-boson-exchange models. II. Hyperonnucleon potential," Physical Review C, vol. 40, pp. 22262245, 1989.

[83] T. A. Rijken, V. G. J. Stoks, and Y. Yamamoto, "Soft-core hyperon-nucleon potentials," Physical Review C, vol. 59, no. 1, p. 21, 1999.

[84] I. R. Afnan and B. F. Gibson, "LL Interaction and ll6He," Physical Review C, vol. 67, no. 1, article 017001, 2003.

[85] L. Ma, Q. Wang, and U.-G. Meißner, "Double heavy trihadron bound state via delocalized $\pi$ bond," Chinese Physics C, vol. 43, no. 1, article 014102, 2019.

[86] S. Moroz and Y. Nishida, "Super Efimov effect for massimbalanced systems," Physical Review A, vol. 90, no. 6, article 063631, 2014.

[87] E. Braaten, C. Langmack, and D. H. Smith, "Born-Oppenheimer approximation for the XYZ mesons," Physical Review D, vol. 90, no. 1, article 014044, 2014.

[88] L. Ma, Q. Wang, and U. G. Meißner, “Tri-meson bound state $B B B *$ via delocalized $\pi \sim$ Bond," Physical Review $D$, vol. 100 , no. 1 , article $014028,2019$.

[89] R. Machleidt, "High-precision, charge-dependent Bonn nucleon-nucleon potential," Physical Review C, vol. 63, no. 2, article 024001, 2001. 
[90] X. Cao, B. S. Zou, and H. S. Xu, "Phenomenological analysis of the double-pion production in nucleon-nucleon collisions up to $2.2 \mathrm{GeV}$," Physical Review C, vol. 81, no. 6, article 065201, 2010.

[91] M. Tanabashi, K. Hagiwara, K. Hikasa et al., "Review of particle physics," Physical Review D, vol. 98, no. 3, article 030001, 2018.

[92] L. Ma, “Tritonlike molecules of three identical baryons," 2020, http://arxiv.org/abs/2005.01131. 